\title{
Development and Attribution of a Linear Referencing System for Managing and Disseminating Traffic Volume Data on Rural Highway Networks
}

\author{
By
}

\begin{abstract}
AUJA OMINSKI
A Thesis submitted to the Faculty of Graduate Studies of

The University of Manitoba

in partial fulfilment of the requirements for the degree of
\end{abstract}

\section{MASTER OF SCIENCE}

Department of Civil Engineering

University of Manitoba

Winnipeg, Manitoba

Copyright () 2018 


\begin{abstract}
Developing and disseminating system-wide traffic volume data are critical objectives of traffic monitoring programs. Jurisdictions commonly use maps to disseminate traffic volume data because maps can easily communicate spatial traffic patterns throughout a highway network. Linear referencing systems (LRSs) are essential in this process, yet little research is available on methods to appropriately sequence a highway network and attribute traffic volume data to these sequences. This research aims to fill this knowledge gap by developing and applying procedures: (1) to segment a linear-referenced highway network into sequences of homogeneous traffic flow; and (2) to attribute traffic volume data to the segmented highway network. The research uses traffic and spatial data collected in Manitoba; however, the procedures are transferrable to other jurisdictions.

This research develops four criteria for segmenting the highway network into sequences based on locations of traffic sources and sinks, such as highway intersections or urban areas. It also develops three multipart principles for attributing traffic data to highway sequences, considering the type of count site, the proximity of the site to the sequence, the recency of data collection, and the presence of traffic sources and sinks. ArcGIS® tools facilitate the iterative consideration of these criteria and principles. The application of the sequencing and attribution procedures enables practitioners to improve the spatial representativeness of a traffic volume map and reveals the importance of evaluating the traffic monitoring program when changes are made to the highway network or sampling program.
\end{abstract}




\section{ACKNOWLEDGEMENTS}

First and foremost, I would like to thank my advisor, Jonathan Regehr, for his constant guidance and support. Somehow even when you were on the other side of the world, I didn't feel like an orphan student. Your passion and enthusiasm is part of what convinced me to try transportation engineering in the first place, which I am grateful for. Thank you for all of the opportunities you have given me, and everything you have taught me (some stuff you probably didn't even realize), both inside and outside the classroom.

Also a thank you to Jeannette Montufar, the other half of the passion and enthusiastic duo that inspired me to pursue a graduate degree in transportation engineering.

To the UMTIG fam (both graduated and not): thanks for making these last two and a half... ok, THREE years, great. All of the lunch hours spent together, the birthdays celebrated, and the ice cream eaten truly made some great memories, so thanks for letting me be a part of that. Special thank you to Karen for helping out with virtually everything. The work you do does not go unnoticed, so thank you!! You also make the best cinnamon buns; just accept it, and don't let anyone tell you differently.

Thank you to Manitoba Infrastructure, including Diana Emerson and Andrew Lindsay, for answering any questions I had throughout my research.

Thank you to my family for supporting me throughout my graduate degree, and to Keagan for always helping in whatever way he could. I'm done now! Hooray!

I would also like to thank the Canadian Transportation Research Forum (CTRF) and the Transportation Association of Canada (TAC) for the financial support they have provided me during the course of my graduate degree. 


\section{TABLE OF CONTENTS}

ABSTRACT

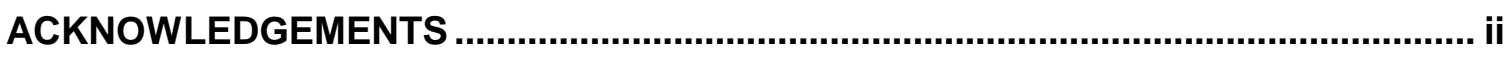

LIST OF TABLES.

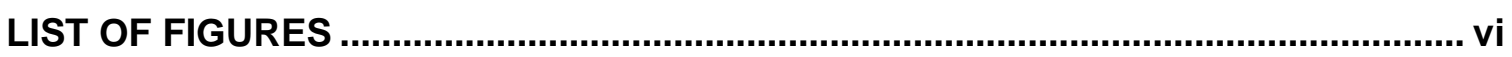

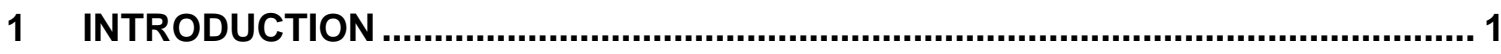

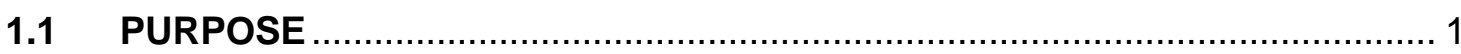

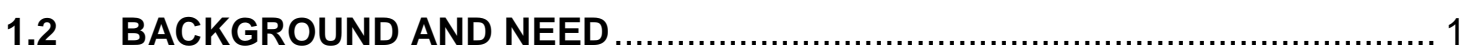

1.3 OBJECTIVES AND SCOPE

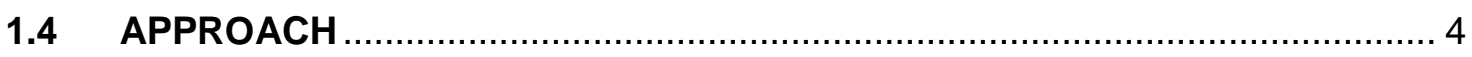

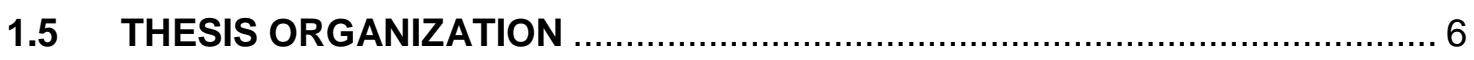

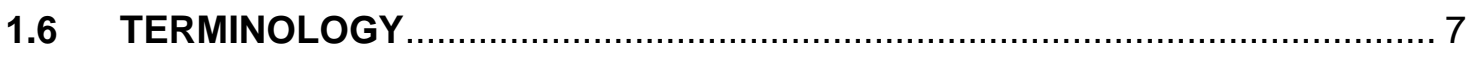

2 LINEAR REFERENCING AND APPLICATIONS IN TRAFFIC MONITORING ...... 10

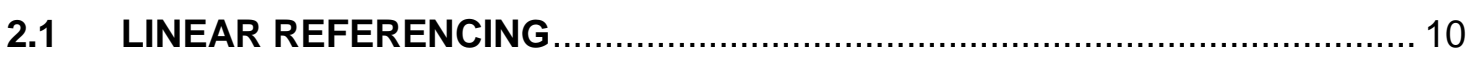

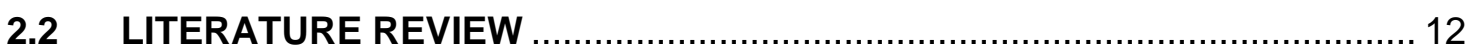

2.2.1 Managing and Disseminating Transportation Data .......................... 13

2.2.2 Managing and Disseminating Traffic Monitoring Data ....................... 16

2.3 TRAFFIC DATA MANAGEMENT \& DISSEMINATION PRACTICES IN

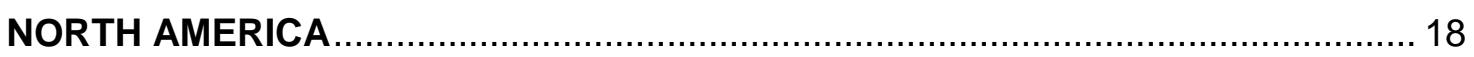

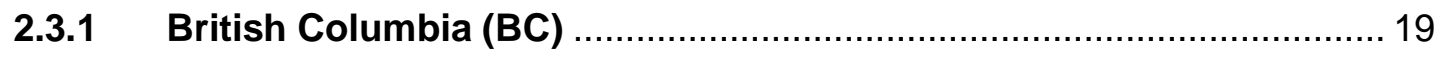

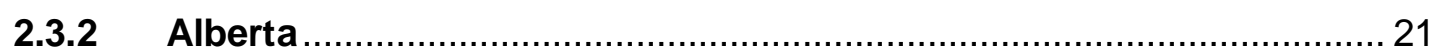

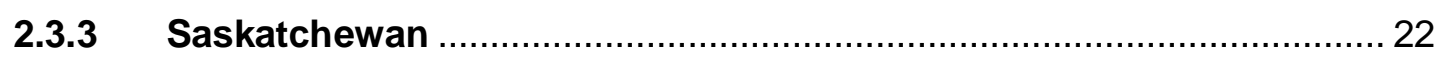

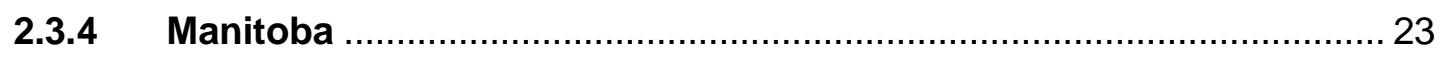

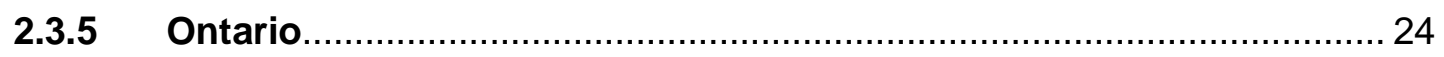

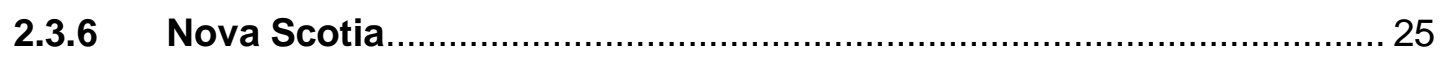

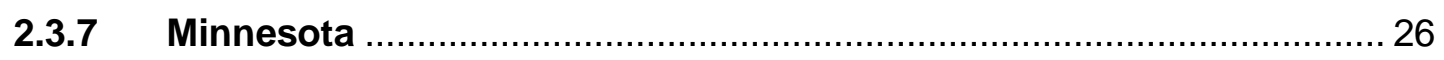

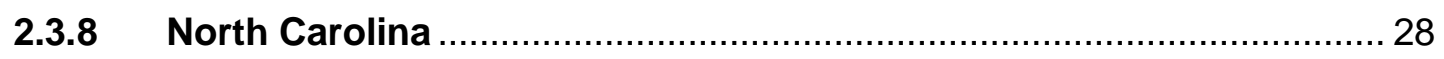

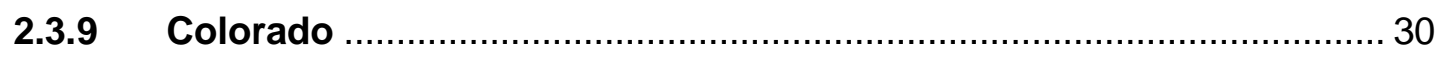

2.4 SUMMARY OF NORTH AMERICAN TRAFFIC DATA DISSEMINATION

TOOLS

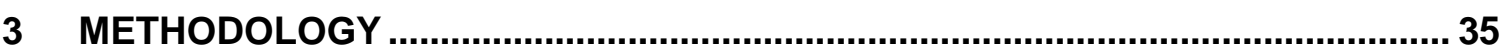

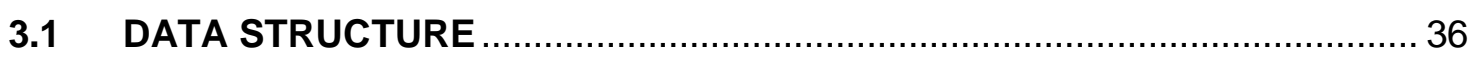

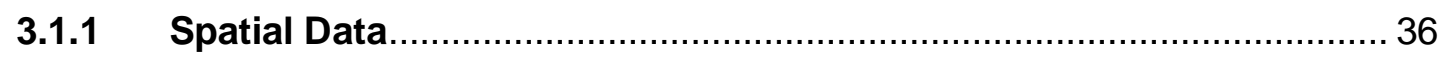

3.1.2 Traffic Count Sites ............................................................................. 43 


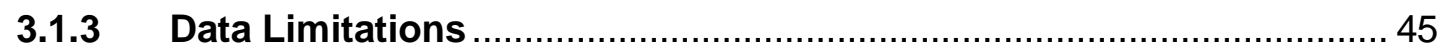

3.2 SEQUENCING PROCEDURE

3.2.1 Sequencing criteria derived from the homogeneity assumption ....... 47

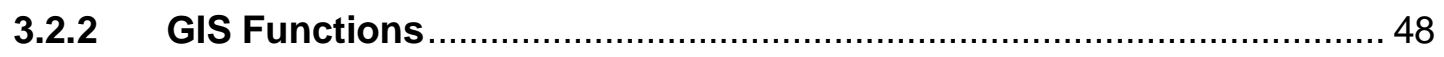

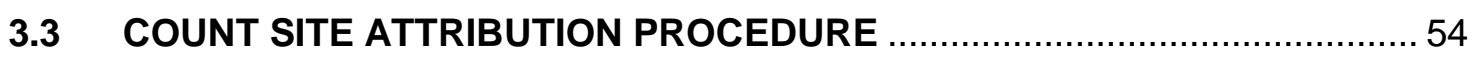

3.3.1 Principles for attributing count sites to sequences ……………….... 55

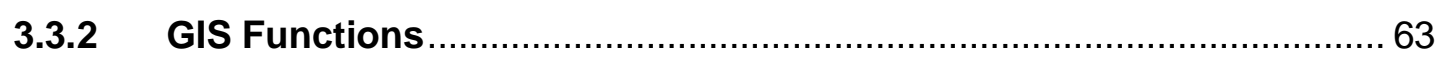

3.4 EVALUATION PROCEDURE AND FINAL STEPS …...................................... 68

3.4.1 Evaluation Procedure .................................................................. 68

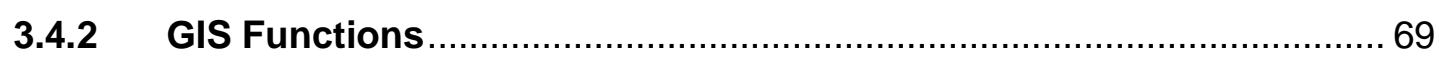

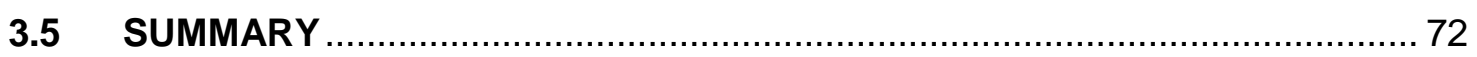

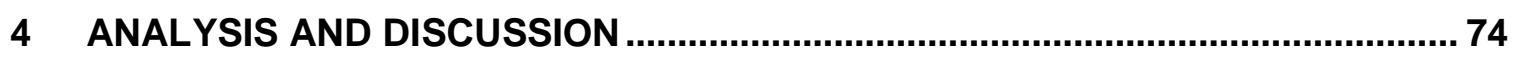

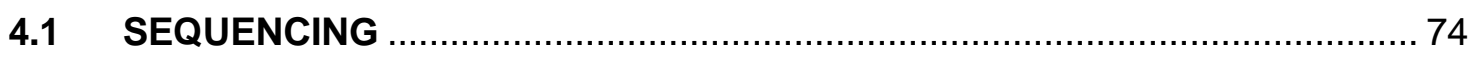

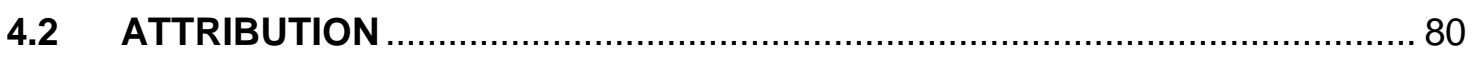

4.3 EVALUATION OF SEQUENCING AND ATTRIBUTION ….......................... 83

4.4 OPPORTUNITIES TO IMPROVE HIGHWAY TRAFFIC DATA COLLECTION 89

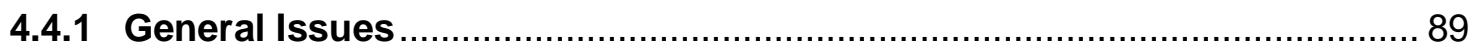

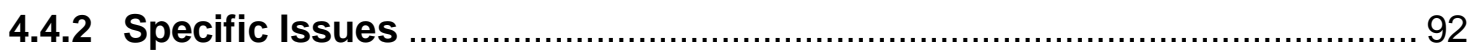

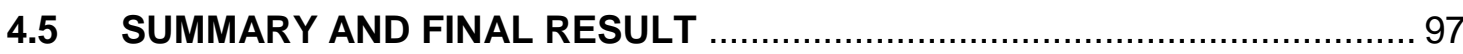

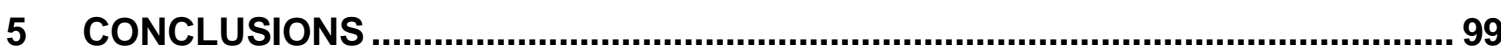

5.1 SUMMARY OF KEY FINDINGS AND IMPLICATIONS.........................................99

5.2 OPPORTUNITIES FOR FUTURE RESEARCH AND WORK IN TRAFFIC

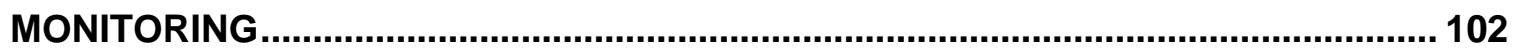

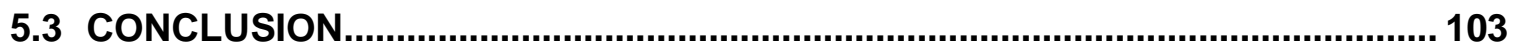

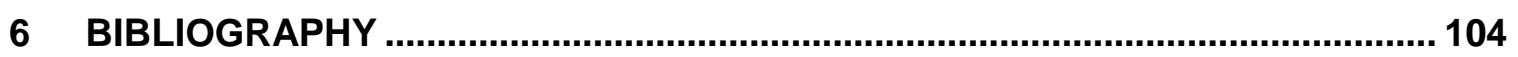

APPENDIX A: FHWA 15-VEHICLE CLASSIFICATION SCHEME ................................ 1

APPENDIX B: SHAPEFILE METADATA

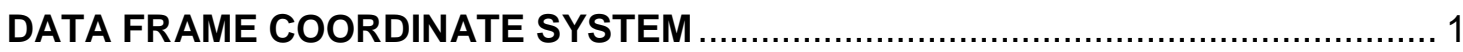

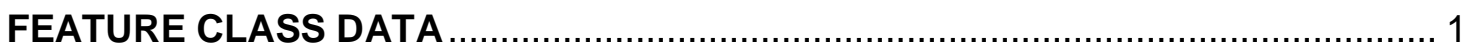

APPENDIX C: PYTHON CODE FOR FIELD CALCULATIONS .................................... 1

START KM FIELD CALCULATOR

APPENDIX D: SEQUENCES FOR IMPROVED TRAFFIC MONITORING ...................... 1

APPENDIX E: REDUNDANT COUNT SITES ……............................................... 1 


\section{LIST OF TABLES}

Table 1: Summary of publicly available traffic data management tools in North America

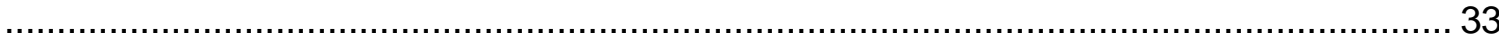

Table 2: Total single-centreline lengths of the three highway types ..............................36

Table 3: Summary of sections by length .................................................................. 39

Table 4: Summary of sequencing procedure ……………………………………...... 74

Table 5: Number of highway/municipal boundary intersection points by city ..................79

Table 6: Summary of highway network by case ......................................................... 80

Table 7: Summary of count site attribution by principle after first round of sequencing

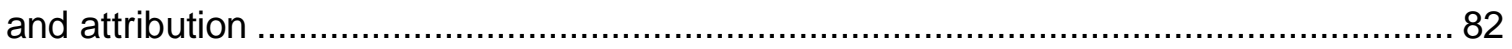

Table 8: Number of sequences by highway type (excluding 600 Series highways) .......85

Table 9: Summary of sequences by length ........................................................... 85

Table 10: Summary of count site attribution by principle after completion of sequencing

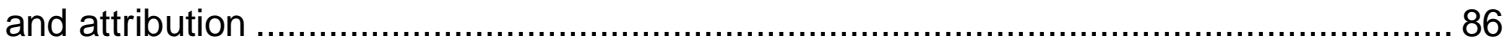

Table 11: Summary of attribution rating by count and length ...................................... 89 


\section{LIST OF FIGURES}

Figure 1: The process of creating a traffic monitoring program as stated in the Traffic Monitoring Practice Guide for Canadian Provinces and Municipalities (Regehr, Poapst, Rempel, Montufar, \& Hallenbeck, 2017).

Figure 2: Referencing point-based data .......................................................... 11

Figure 3: Referencing line-based data ................................................................. 12

Figure 4: BC Ministry of Transportation and Infrastructure traffic data dissemination tool

(BC Ministry of Transportation and Infrastructure, 2017) ............................................. 20

Figure 5: Alberta Ministry of Transportation traffic data dissemination tool (Alberta Ministry of Transportation, 2017) .................................................................... 22

Figure 6: Manitoba's traffic data dissemination tool (Manitoba Highway Traffic

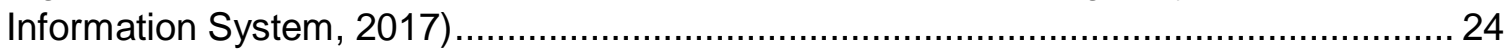

Figure 7: Ontario Ministry of Transportation iCorridor traffic data dissemination tool (Ontario Ministry of Transportation, 2010) …………….................................. 25 Figure 8: Example of sequencing highway network to create a 1:1 ratio of count sites to

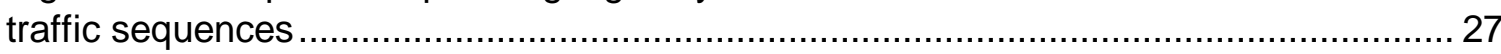

Figure 9: Minnesota Department of Transportation traffic data dissemination tool

(Minnesota Department of Transportation, 2017) ...................................................... 28

Figure 10: North Carolina Department of Transportation traffic data dissemination tool (North Carolina Department of Transportation, 2016) .............................................. 30

Figure 11: Colorado Department of Transportation traffic volume data dissemination tool

(Colorado Department of Transportation, 2017) ………………………………..... 31

Figure 12: Schematic depiction of the sequencing, attribution, and evaluation procedures

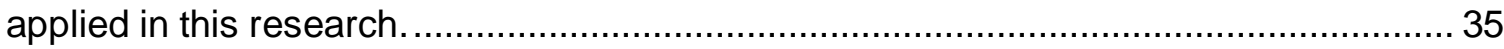

Figure 13: Manitoba's Provincial Trunk Highways, Provincial Roads, and 600 Series

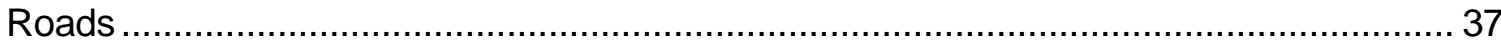

Figure 14: Ml's double-centreline representation of the Manitoba highway network .......38

Figure 15: Example of traffic control sections defined by MI .......................................... 39

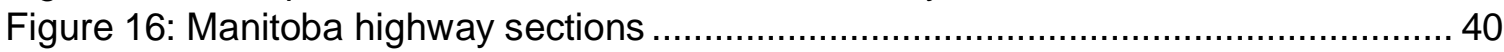

Figure 17: Illustration of Control Section numeration ................................................ 41

Figure 18: Example of highway sections further divided into highway sequences to allow

for the homogeneity assumption .......................................................................... 42

Figure 19: Continuous and short-duration count sites in Manitoba .............................. 45

Figure 20: Flowchart of sequencing procedure....................................................... 49

Figure 21: Schematic of the single-centreline representation of the highway network ...50

Figure 22: Illustration of creating the point layer at highway intersection locations ........51

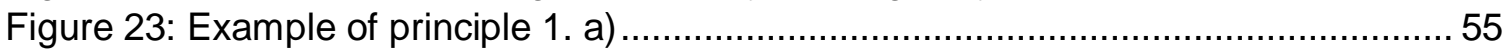

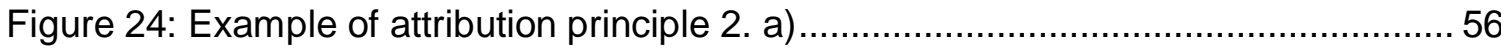

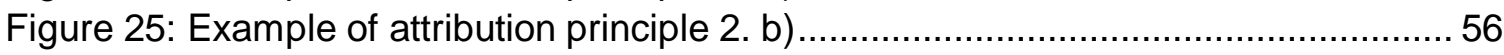

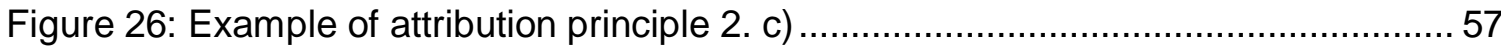

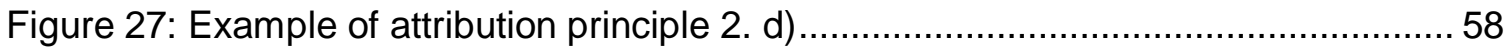

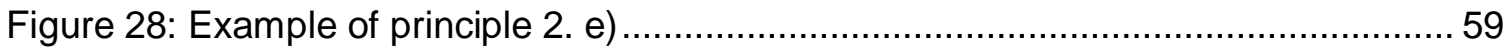

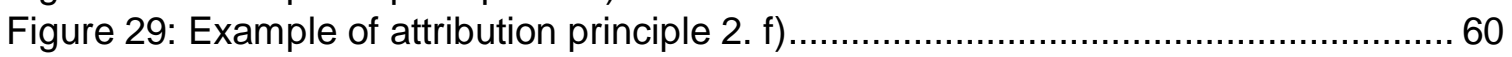

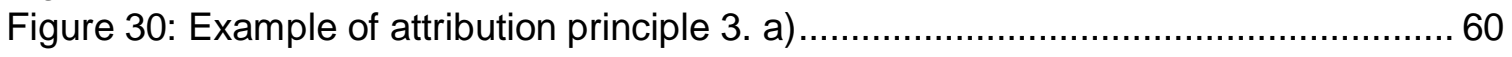

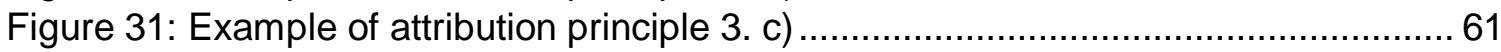

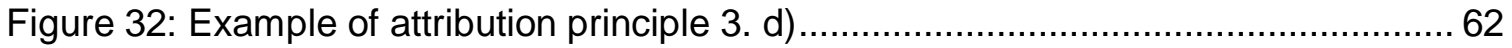




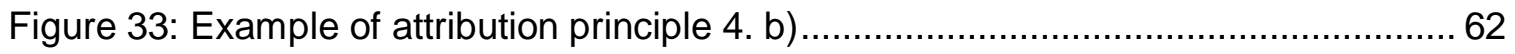

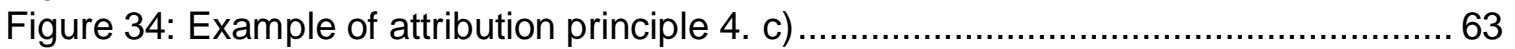

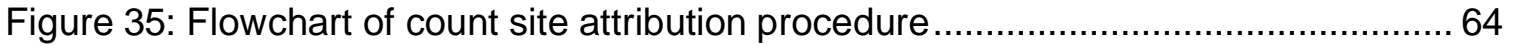

Figure 36: Error in snapping counts to nearest highway on the highway network ..........66

Figure 37: Flowchart of evaluation procedure........................................................ 70

Figure 38: Sequenced highway network after first stage of sequencing ......................... 75

Figure 39: Highway intersections represented as points (Criterion 1 \& 2) ...................... 77

Figure 40: Municipal road/highway intersection locations (Criterion 3) .......................... 78

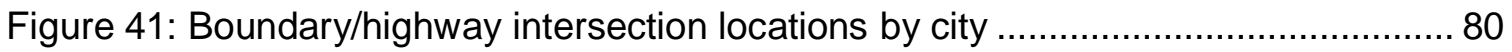

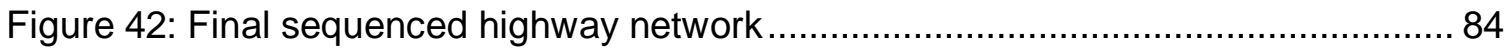

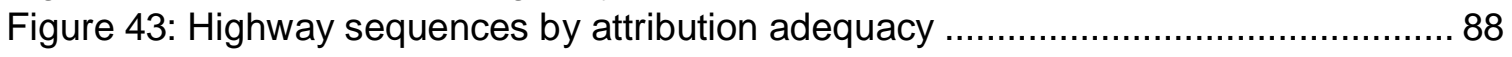

Figure 44: Illustration of built up area inside an urban boundary ................................... 92

Figure 45: Highway sequences on the North and South Perimeter lacking data collection

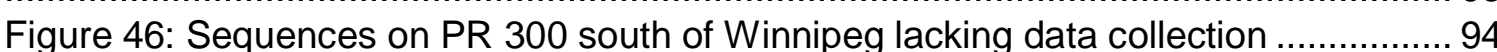

Figure 47: Bridge out of service near Wawanesa, MB, introducing a highway sequence

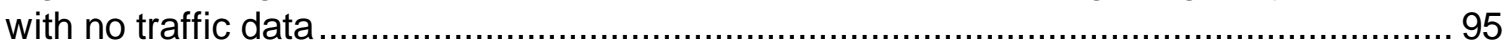

Figure 48: PR 207 sequences in Ste. Anne lacking data collection ............................... 96

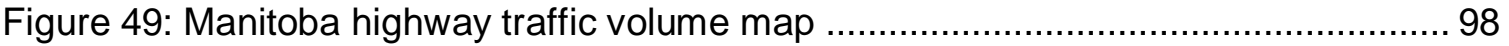




\section{INTRODUCTION}

\subsection{PURPOSE}

The purpose of this research is to develop a linear referencing system (LRS) to manage and disseminate system-wide traffic volume data. Public transportation agencies collect traffic data at sites (points) across a highway network. An LRS is a tool to manage and disseminate these data, and integrate it with other types of transportation data. To accomplish this, the LRS must be subdivided into segments, which are assumed to have constant, or homogeneous, traffic volume characteristics. This is referred to as the homogeneity assumption. The attribution of site-specific traffic volume data to segments depends on the number and location of data collection sites in a jurisdiction, its sampling plan, and available resources. This research develops and applies criteria for segmenting an LRS and attributing data to segments within the context of a rural highway network. In so doing, it contributes new knowledge to support more efficient management of traffic data and more representative data visualizations.

\subsection{BACKGROUND AND NEED}

With technological advancements and the digitalization of data, the volume of transportation data being collected has increased substantially over the past several years (Gharaibeh, Oti, Schrank, \& Zmud, 2017). Greater volumes of transportation data offer benefits to many aspects of transportation, including monitoring performance and assisting in planning and design. Simultaneously, data should be accessible in a way that is beneficial to data customers (Gharaibeh, Oti, Schrank, \& Zmud, 2017). The need for accessible and representative data is particularly relevant in the public sector, where tax dollars are used to collect data to improve public infrastructure, and failing to meet 
this need can cause a jurisdiction to lose credibility (Federal Highway Administration, 2016).

Linear referencing, or a linear referencing system (LRS), is a method of describing locations of features or spatial data based on their distance from a known starting point (Olsen, Raugust, \& Roe, 2013). An LRS is commonly used for data integration, analysis, visualization, and dissemination. Roads, railways, and other transportation infrastructure are typically linear, or can be represented as such; therefore, linear referencing works well for managing different forms of transportation data.

Linear referencing and GIS software are increasingly common for managing traffic data and providing these data to customers (Olsen, Raugust, \& Roe, 2013). Traffic monitoring programs provide vital data for almost all tasks performed by a highway agency (Federal Highway Administration, 2016), (Regehr, Poapst, Rempel, Montufar, \& Hallenbeck, 2017). For example, AADT and vehicle kilometres traveled (VKT) are necessary to develop safety performance functions (SPFs) and determine collision rates, which are common tools for assessing safety. Similarly, annual average daily truck traffic (AADTT), vehicle class distributions (VCDs), and axle load spectra (ALS) are critical for designing and evaluating pavements. With the wide variety of data customers and needs, traffic data should be easily accessible and understood. Considering the wide variety of data users, it is also important for the traffic data to be representative of reality, both in value and in its geographical variation (Gharaibeh, Oti, Schrank, \& Zmud, 2017).

Effective management and dissemination of traffic volume data necessitates special accommodations within an LRS. Consider a common method of traffic data dissemination - a traffic volume map. Data customers like to know where and how much traffic volume is occurring across a network, often in the form of AADT, or VKT (Regehr, Poapst, Rempel, Montufar, \& Hallenbeck, 2017). In creating a traffic volume map, point- 
based traffic counts must be attributed to line-based highway segments. To do so, highway segments with homogeneous traffic volume characteristics must be defined, and a process to attribute traffic volume data to these segments must be created. This network segmentation and data attribution is also key in calculating network vehicledistance travelled, often measured as vehicle-kilometres travelled in Canada, which is necessary in understanding network utilization. However, current North American guidance, including the AASHTO Guidelines for Traffic Data Programs (American Association of State Highway and Transportation Officials, 2009), the Traffic Monitoring Guide (Federal Highway Administration, 2016), and the Traffic Monitoring Practices Guide for Canadian Provinces and Municipalities (Regehr, Poapst, Rempel, Montufar, \& Hallenbeck, 2017), does not discuss defining segments of homogeneous traffic volume and attributing traffic data to these segments in detail. This research aims to fill this knowledge gap.

\subsection{OBJECTIVES AND SCOPE}

The objectives of this research are:

1. to review background information related to linear referencing, including best practices of current linear referencing systems;

2. to define criteria and principles for segmenting and attributing data to an LRS;

3. to use GIS tools to apply these criteria and principles in the development of an LRS for managing and disseminating traffic volume data collected in Manitoba; and

4. to analyze and summarize results, including segmentation and attribution statistics, and identify opportunities to optimize data collection on the highway network. 
The scope of this research is constrained as follows:

- The data considered are rural; no urban data are used.

- Spatial data are provided by Manitoba Infrastructure (MI) in a shapefile format. The traffic volume data are gathered by $\mathrm{MI}$ and processed by graduate students working on the Manitoba Highway Traffic Information System (MHTIS).

- This research uses the ArcGIS® suite of tools to create the LRS. ArcMap® is used to segment the road network, assign specific traffic counts (whether shortduration or permanent counts) to segments, and create the spatial buffer around major population centres.

- The accuracy of the traffic volume data is not the focus of the research; rather the focus is the ability of the LRS to disseminate the data adequately.

- This research develops sequencing and attribution procedures that consider total traffic volumes (including passenger vehicles and trucks). These procedures would require adjustment if volumes were disaggregated by vehicle class.

While the data used are from rural Manitoba highways, the methodology of the research may be applied elsewhere.

\subsection{APPROACH}

The Traffic Monitoring Practices Guide for Canadian Provinces and Municipalities describes a traffic monitoring program in five components: (1) design, (2) collection, (3) analysis, (4) dissemination, and (5) evaluation. Figure 1 shows this process. 


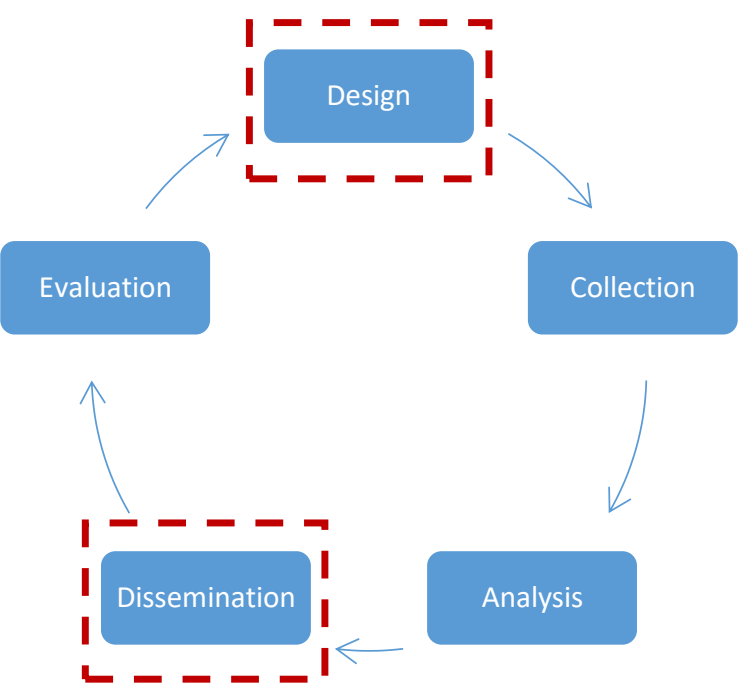

Figure 1: The process of creating a traffic monitoring program as stated in the Traffic Monitoring Practice Guide for Canadian Provinces and Municipalities (Regehr, Poapst, Rempel, Montufar, \& Hallenbeck, 2017).

The first component, design, involves the planning of the traffic monitoring program. It considers data customers and their needs, and how a jurisdiction may obtain systemwide data collection coverage. The collection component considers methods of data collection, including the selection of appropriate data collection equipment based on the needs of the traffic data customer. Analysis involves data validation and summarization, including traffic statistics obtained from data collection equipment. The dissemination component encompasses traffic data reporting, and considers how customers will access the data. Finally, the evaluation component evaluates the performance of the data collection program and considers how it may be improved (Regehr, Poapst, Rempel, Montufar, \& Hallenbeck, 2017). Evidently, the process is cyclic, as the evaluation component allows for program redesign.

This research particularly contributes to the design and dissemination components of a traffic monitoring program, and discusses the implications of data dissemination on the design of a traffic monitoring program. 
This research creates an LRS of the Manitoba highway network for the purpose of managing and disseminating traffic data, considering: (1) where traffic volume homogeneity can be assumed for highway sequencing, and (2) how count stations and their traffic data can be attributed to the network to produce a representative traffic volume map. To create homogeneous traffic volume sequences, the research applies knowledge of traffic volume patterns and locations of traffic volume change, including at: (1) Provincial Trunk Highway (PTH)/Provincial Road (PR)/600 Series intersections, (2) municipal road intersections, and (3) urban boundaries. Similarly, the attribution of count sites to highway sequences considers: (1) the type of count site and accuracy of its data, (2) the proximity of the count site to a highway sequence, (3) the recency of data collection, and (4) the presence of traffic sources and sinks.

\subsection{THESIS ORGANIZATION}

This thesis comprises five chapters, including this introductory chapter.

Chapter 2 - Literature and Practice Review: This chapter discusses the use of LRS for various purposes, including the management and dissemination of traffic volume data. It also describes various methods and considerations used in creating an LRS and investigates linear referencing in highway traffic monitoring across the country.

Chapter 3 - Research Methodology: This chapter discusses the design of the LRS and the attribution of traffic volume data to the LRS; this includes a discussion of design criteria, and the GIS tools used to create the LRS.

Chapter 4 - Results and Analysis: This chapter summarizes the results of creating the LRS, including describing the sequencing and attribution statistics, and expanding on the implications of the findings such as optimizing count locations based on the LRS. 
Chapter 5 - Conclusions: This chapter concludes the thesis by presenting key findings and the implications of these findings. This chapter also discusses research limitations and makes recommendations for future research.

\subsection{TERMINOLOGY}

The following are terms used throughout this research:

Annual Average Daily Traffic (AADT) - The number of vehicles passing a point on a road segment during an average day of the year.

Attribute Data - Supplemental information appended to spatial features (such as the number or surface type of highways) that is available in the attribute table of a layer of spatial features.

Automatic Vehicle Classifier (AVC) - A type of continuous count that counts passing vehicles, as well as collects speed, classification, and axle spacing data for those vehicles.

Base Map - The underlying imagery and/or data of a map.

Continuous Count - A count site embedded in the pavement that collects data 24 hours per day, 365 days of the year. Also sometimes referred to as an Automated Traffic Recorder or ATR.

Control Section (or Section) - A defined segment of a highway used by Manitoba Infrastructure (MI) for spatial referencing.

Feature - A point, line or polygon with spatial characteristics and associated data. 
Field - A column in an attribute table that contains text or numeric information about a spatial feature. An example of a field would be Highway Number, where the field would contain the highway number of all spatial features in the table.

Homogeneous (Homogeneity Assumption) - A pragmatic simplification that disregards immaterial changes in traffic volume along a highway. Its application underpins the development of criteria used in the sequencing procedure, which in turn facilitates the attribution of count sites to sequences.

Linear Referencing System - A method of describing feature locations as a distance along some linear element.

Permanent Count Station (PCS) - A type of continuous count that counts passing vehicles.

Provincial Road (PR) - A secondary highway in Manitoba.

Provincial Trunk Highway (PTH) - A primary highway in Manitoba.

Sequence - A subdivision of a control section along which traffic volume is assumed to be homogeneous.

Shapefile - An electronic file of one or more similar spatial features, such as points, lines, or polygons.

Short-Duration Count - A site where traffic data are collected for a period of 48 hours twice during the survey year, which occur every three years. Also sometimes referred to as a Coverage Count Station or CCS. 
Town Count - A type of short-duration count collected within city/town boundaries. For the purpose of this research, town counts are considered separate from short-duration counts.

Weigh-in-motion (WIM) - A type of continuous count that counts passing vehicles, as well as collects speed, classification, axle spacing, and axle weight data for those vehicles

600 Series Highway - A tertiary highway in Manitoba. 


\section{LINEAR REFERENCING AND APPLICATIONS IN TRAFFIC MONITORING}

This chapter introduces the concept of linear referencing and then reviews literature regarding the application of linear referencing for transportation and traffic data management and dissemination.

This chapter also summarizes publicly available traffic data management and dissemination tools used by North American jurisdictions, including most Canadian provinces and three American states.

\subsection{LINEAR REFERENCING}

Linear referencing, or a linear referencing system (LRS), is a method of describing locations of features or spatial data based on their distance along a linear element (ESRI, 2016). LRSs are commonly used for transportation data management. Since roads, railways, and other transportation infrastructure are typically linear, or can be represented as such, linear referencing works well for managing different forms of transportation data. Types of transportation data visualized in linear referencing are point- and line-based data.

\section{Point-Based Data}

Point data are data referenced to a particular geographic coordinate, whether this is a location of an event, a particular asset, or a landmark. There are different ways point data can be represented based on the type of data and how they are collected.

Point data can be represented either as individual points along the line they are being referenced to, or as individual points some distance away from a defined location on the 
line. Figure 2 shows these two possible point representations. Examining Figure 2, the turquoise point can be described at a location "2" on the line, or the purple point can be represented as a point 3 units away from the location "20".

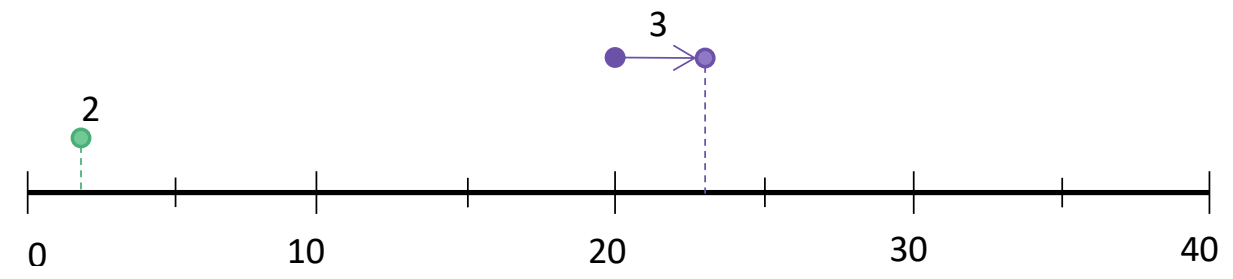

Figure 2: Referencing point-based data

These two methods of describing the location of point data are beneficial in different situations. As an example, road incidents may be measured at a location some distance away from a major intersection or landmark, and so the method used to describe the yellow point would be of benefit. In contrast, if one considers the location of a particular asset and uses a GPS to find the location of that asset, it may be represented similar to the green point.

\section{Line-Based Data}

Line data are data of the same value over some distance, such as traffic volume between two intersections or data on road surface condition. Similar to point data, line data can be represented in two different ways based on the data collection method and the nature of the data.

Line data can be represented as individual lines of a particular value between two locations, or as individual lines starting at a particular location and continuing a given distance in a certain direction. Figure 3 shows these two methods of representing linebased data. 


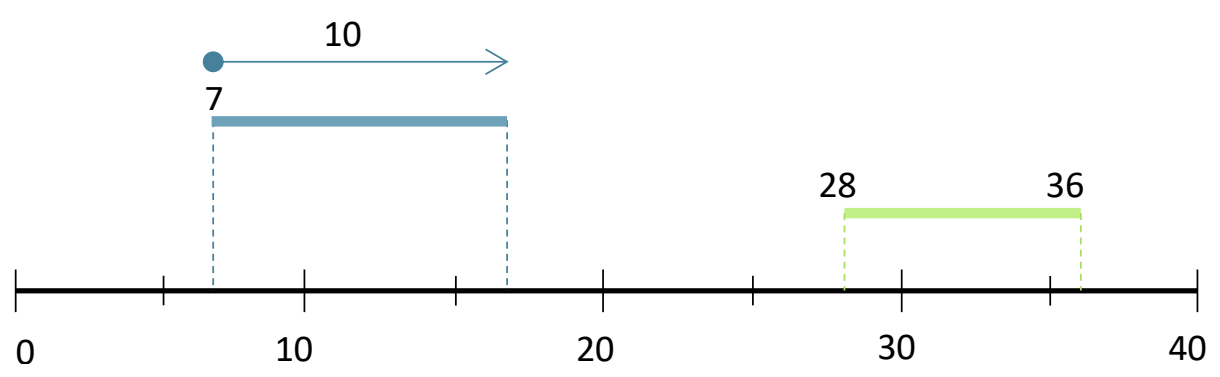

Figure 3: Referencing line-based data

As shown in the figure, the green line is represented as existing between locations "28" and " 36 ", whereas the blue line is represented as starting at location " 7 " and continuing east for 10 units.

These two methods of describing the location of line-based data are beneficial in different situations. As an example, traffic volume may be considered homogeneous between two intersections, and can be represented in a similar fashion to the orange line in Figure 3. On the other hand, road surface condition may start at an intersection and continue for a certain distance in a given direction, and may be best represented in a method similar to the blue line. Both data representations are an option and can be chosen based on the needs of data collection and analysis.

\subsection{LITERATURE REVIEW}

This section discusses the application of LRS for: (1) managing and disseminating transportation data, and (2) managing and disseminating traffic data. Four documents are particularly relevant for this review:

- Traffic Monitoring Practices Guide for Canadian Provinces and Municipalities,

- NCHRP Research Report 446: Use of Advanced Geospatial Data, Tools, Technologies, and Information in Department of Transportation Projects, 
- NCHRP Research Report 865: Guidance for Development and Management of Sustainable Enterprise Information Portals, and

- The Transportation Research Circular Number E-C227: Advancing Highway Traffic Monitoring Through Strategic Research.

Additional literature supplements the findings in these documents. The findings are summarized at the end of this section.

\subsubsection{Managing and Disseminating Transportation Data}

Transportation data, in their many forms, are vital to bettering transportation systems through informed decision-making (Gharaibeh, Oti, Schrank, \& Zmud, 2017). With technological advancements, transportation data collection has substantially increased in both volume and complexity over the past several years, yet the quantity of data maintained by public agencies is often unknown to the agencies themselves (Gharaibeh, Oti, Schrank, \& Zmud, 2017). This volume of data has the potential to benefit many aspects of transportation, including monitoring performance, and assisting in planning and design. However, a key concern for public agencies is ensuring that data are accessible (Gharaibeh, Oti, Schrank, \& Zmud, 2017), and for this benefit to be realized, the data must be managed, disseminated, and visualized for data customers. This is particularly important from a public perspective, where public funds are used to collect traffic data to improve public infrastructure, and failing to meet this need can cause a jurisdiction to lose public support (Federal Highway Administration, 2016).

A common method of visualizing and analyzing various types of transportation data is with GIS. This method is increasingly becoming a preferred method for such tasks (Applied Engineering Management Corporation, 2018). Using advanced geospatial technologies allows for access to data across sectors, and can introduce significant cost 
savings in minimizing duplicate data collection and enabling informed decision-making and quality management (Olsen, Raugust, \& Roe, 2013).

Public agencies around the world have transportation data that require managing and disseminating to allow data customer access. All areas of transportation, including safety, operations, transit, and asset management, have data that are essential to improving transportation systems. There are numerous methods of gathering, managing, and disseminating traffic data, which are ideal in different circumstances. Recent examples for each of these areas of application follow.

- Safety: In Iran, researchers were able to utilize GIS to analyze and visualize crash data in order to determine high crash density locations, considering collision frequency and severity (Rouzbeh \& Shahriar, 2017).This information facilitates future decision making to improve safety at such locations. Similarly, California utilizes linear referencing in a system displaying vehicle collisions (Bingham \& Kang, 2013) and Brazil utilizes GIS for collision investigation (Berté, de Souza, \& Leitão, 2016).

- Operations: The Virginia DOT (VDOT) utilizes a web tool that allows residents to view a map of snow clearing, including where snowplows have been and where they are going. This particular tool was developed using Microsoft software, including: Internet Information Services web server, ASP.Net web framework, MapPoint mapping framework, Bing maps online map service, and Azure blob storage cloud storage (Applied Engineering Management Corporation, 2018).

- Transit: The once unmapped transit network in Bogota, Colombia, was recently mapped to show all routes in the city (Goldwyn \& Vergel-Tovar, 2018). They were able to map the network using GPS data from smartphones, and use Python to merge GPS traces with origin route information. The routes also had 
associated data, including average travel speed and fares. They now have the opportunity to merge these datasets with other spatialized datasets in Bogota, which will help inform future transportation decisions and allow them to track their progress to providing access to transport systems for all citizens. Similarly, XuChang, China, utilizes linear referencing to display bus lines and stops, and allow for user querying (Shuna \& Changpo, 2012). Additionally, Beijing utilizes GIS to visualize transit performance metrics, including speed, travel-time reliability, ridership, and headway (Ma \& Wang, 2014).

- Asset Management: Queensland Rail (QR) in Queensland, Australia, wanted to streamline the traditional exchange of rail asset data via as-built drawings and other physical items (Burdett, 2010). To complete this goal, they pursued an LRS that would provide asset information online rather than as a physical copy. In creating an LRS, QR was able to improve their exchange of asset data, and a framework for the delivery of such data was created. The LRS allowed QR to display data from many disciplines, including signals, stations, and track. In another example, North Carolina utilizes GIS to visualize existing and planned bicycle and pedestrian infrastructure (Stull, Jackson, \& O'Brien, 2017). Finally, in Tarrant County, Texas, GIS is utilized for pipeline asset management, with the ability to record pipeline condition and prioritize pipeline repair or replacement (Nardini, Zarghamee, Bain, Jalbert, \& Remer, 2015)

Evidently, there are numerous methods to manage and disseminate traffic data, and many areas of transportation have a need for a method of managing and disseminating the data they gather. 


\subsubsection{Managing and Disseminating Traffic Monitoring Data}

The field of traffic monitoring offers its own intricacies and complications in data management and dissemination. A substantial amount of traffic data are collected on a daily basis due to the nature of the data and the data collection process. Certain data, such as traffic volume data, are collected at discrete points on a highway network, similar to crash data. Continuous count sites collect data of all passing vehicles 24 hours per day, 7 days per week, 365 days per year, with the exception of instances of equipment malfunction. Short-duration counts are typically between 24 hours and 7 days in length (Stolz, 2017) and collect data on all passing vehicles during that time. They are performed to fill in the network gaps, and obtain spatial variations in traffic volume (Regehr, Poapst, Rempel, Montufar, \& Hallenbeck, 2017).

The most important goal of a traffic monitoring program is to obtain system-wide coverage (Regehr, Poapst, Rempel, Montufar, \& Hallenbeck, 2017), which becomes a complex task given the extent of transportation systems and point-based methods of data collection. Jurisdictions do not have the resources or funding to continuously monitor all traffic patterns for an entire road network. There are numerous methods to obtain system-wide coverage, all of which involve collecting data at points on traffic segments. These methods include the following:

1. Periodically sampling all highway segments in the network, be that continuously or for a short-duration once every specified number of years (e.g., three).

2. Applying a hierarchical approach, in which data are applied to a segment as either values specific to that site, values from a site with similar traffic patterns, or values assumed based on jurisdictional defaults. 
3. Stratified random sampling, where the highway network is divided into groups and segments from each of those groups are sampled (Regehr, Poapst, Rempel, Montufar, \& Hallenbeck, 2017).

Regardless of the method, these segments should have minimal traffic fluctuation, which can also be referred to as homogeneous traffic volume, to ensure data are representative of actual conditions (Regehr, Poapst, Rempel, Montufar, \& Hallenbeck, 2017). This implies that a network should comprise many short traffic segments. However, the more segments there are, the more difficult system-wide coverage becomes. At the same time, in determining these traffic segments, it may be unknown what traffic fluctuations exist and where they exist if limited data are available.

Moreover, there is a desire for traffic data (namely traffic volume data) to be disseminated in the form of a traffic volume map. This means the discrete point estimates of traffic volume are attributed to road segments, which differs from other forms of point-based data. A traffic volume map is an easy to understand dissemination tool, which is ideal for the variety of traffic data customers, including those in the public, private, and academic sectors, as well as members of the public with non-technical backgrounds (Regehr, Poapst, Rempel, Montufar, \& Hallenbeck, 2017). Another key outcome of attributing point-based traffic volumes to highway segments is to produce network vehicle-distance travelled (VDT) (or vehicle-kilometres travelled, VKT), which is an important metric for measuring safety (e.g., collisions per 100 million VKT). VDT is also a necessary measure in highway design, including pavements and highway geometry (Regehr \& Reimer, 2013). VKT is calculated using the following formula.

$$
V K T=\sum(A A D T \times d \times 365)
$$

where: 
VKT $=$ vehicle-kilometres travelled

AADT = annual average daily traffic

$d=$ distance or length of the highway segment

Evidently, both segment AADT and segment length play a key role in the calculation of VKT, which implies a need for a standard method of segmenting a highway network and attributing data to the defined segments. The AASHTO Traffic Data Program Guidelines defines a traffic segment as "A unit of roadway on which traffic volumes are reasonably homogeneous... [that] may be subdivided into short segments... on the basis of changes in political jurisdiction or changes in road characteristics" (American Association of State Highway and Transportation Officials, 2009). The Traffic Monitoring Guide suggests volumes on traffic segments should be within plus or minus $10 \%$ of each other (Federal Highway Administration, 2016). Finally, the Traffic Monitoring Practices Guide for Canadian Provinces and Municipalities states common locations for nodes between traffic segments are at intersections, boundaries between rural and urban areas, jurisdictional boundaries, and traffic origins/destinations (Regehr, Poapst, Rempel, Montufar, \& Hallenbeck, 2017). However, according to existing guidance in North America, a common methodology of creating traffic segments and attributing data to these segments does not exist (American Association of State Highway and Transportation Officials, 2009), (Federal Highway Administration, 2016), (Regehr, Poapst, Rempel, Montufar, \& Hallenbeck, 2017).

\subsection{TRAFFIC DATA MANAGEMENT \& DISSEMINATION PRACTICES IN NORTH AMERICA}

Many jurisdictions in North America have utilized GIS and linear referencing for the purposes of traffic data management and dissemination. This section outlines publicly 
available traffic data dissemination tools for Ontario, Alberta, British Columbia, Saskatchewan, Manitoba, and Nova Scotia in Canada. The goal was to summarize traffic data dissemination tools for all Canadian provinces; however, provinces that do not have publicly available tools and did not respond to being contacted are not included. To supplement the Canadian review, three U.S. jurisdictions (Minnesota, North Carolina, and Colorado) known to have advanced traffic monitoring programs were also included.

Data was gathered by reviewing publicly available online sources for each of the above jurisdictions. These online data sources were reviewed for particular capabilities and information, including availability of key data such as traffic volumes, count stations, and traffic segments, as well as to obtain an idea of how the highway networks were segmented for traffic volume attribution, and how that traffic volume was attributed to the network.

\subsubsection{British Columbia (BC)}

The Ministry of Transportation and Infrastructure in BC displays traffic data in their Traffic Data Program GIS Application (BC Ministry of Transportation and Infrastructure, 2017). This Bing-based tool displays the province's permanent count sites, short duration count sites, turning movement counts, and uniform traffic volume segments (UTVS), and allows access to these data. Annual average daily traffic (AADT) and summer average daily traffic (SADT) data are accessible either at particular count locations or by selecting a UTVS. The UTVSs have varying colours from blue to yellow to highlight segmentation breaks.

UTVSs are highway segments of varying length assumed to have consistent traffic patterns. They are each attributed a Traffic Measurement Site (TMS) and corresponding 
most recent AADT and SADT. UTVSs also have historical growth factors of 1, 3, and 10 years, and an approximate confidence level associated with a given growth factor as well as how the growth factor was determined.

The map has four layers - 2004-Present Traffic Data, 1994-2003 Traffic Data, Turning Movement Counts and Uniform Traffic Volume Segments (UTVS) - that can be turned on and off by the user, as well as the option to view the data on either a Bing Roads or a Bing Hybrid base map. The tool also includes a search box that allows the search for locations on the map.

Users can download traffic data reports of each station, including AADT, SADT, and vehicle classification data (at permanent count sites), as a pdf, or view reports online. Users may also download a zip file of intersection turning movement count data containing .xlsx files of the turning movement counts in 15-minute bins, as well as summarized daily traffic volume and peak hour period volume data. Figure 4 gives an overview of the tool.

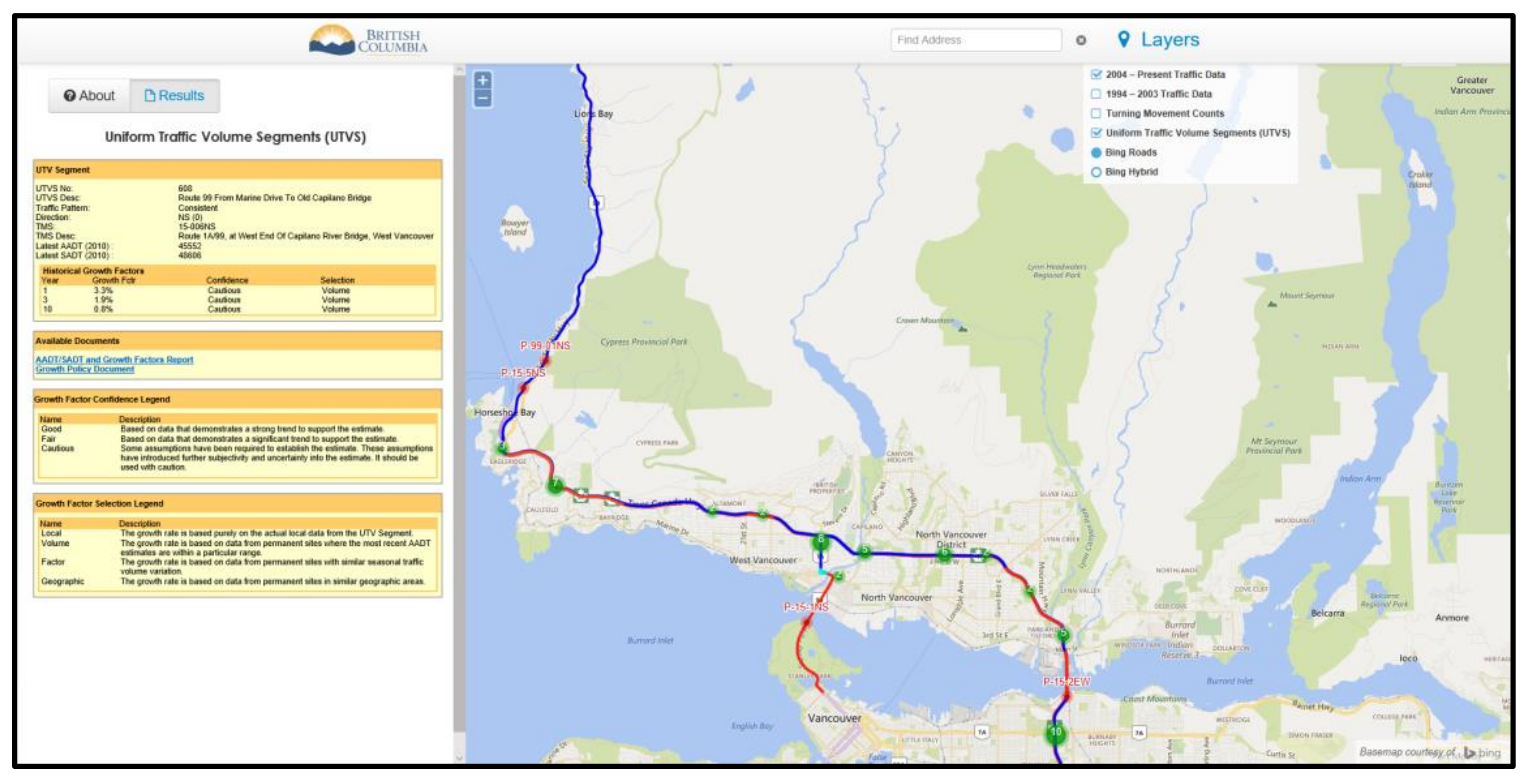

Figure 4: BC Ministry of Transportation and Infrastructure traffic data dissemination tool (BC Ministry of Transportation and Infrastructure, 2017) 


\subsubsection{Alberta}

The Government of Alberta Ministry of Transportation utilizes a Google-based map tool for the purpose of data dissemination (Alberta Ministry of Transportation, 2017). This tool allows access to traffic data and statistics, including intersection turning-movement counts, hourly automated traffic recorder (ATR) counts, and traffic control section specific data such as Weighted Annual Average Daily Traffic (WAADT) and Equivalent Single Axle Load (ESAL)/day/direction.

Traffic control sections are highway segments of various length with assumed homogeneous traffic volume. One or more traffic control sections comprise a control section; each traffic control section has a unique combination of a highway number, a two-digit control section and a two-digit traffic control section. The line thickness of a traffic control sections signifies the quantity of traffic volume on that segment; thicker lines represent locations of greater volume whereas thinner lines represent areas of lesser volume.

The map has three layers - traffic control sections, automated traffic recorders, and intersections - that can be turned on an off by the user. The tool also includes a search box that allows the search for locations on the map.

Users can download hourly volume data from ATRs in an .xIsx file format for the past 10 years, or viewed online by month of year (Alberta Ministry of Transportation, 2017). A monthly summary of traffic volumes is also available for past years. Similarly, users can download intersection turning movement counts in an .xlsx or .pdf file format, or view them online. Two pre-set data figures are also available for each traffic control section when selected. Additional AADT data dating back to 1962 and weigh-in-motion (WIM) 
reports are available for download from the Alberta Transportation website. Figure 5 gives an overview of the tool.

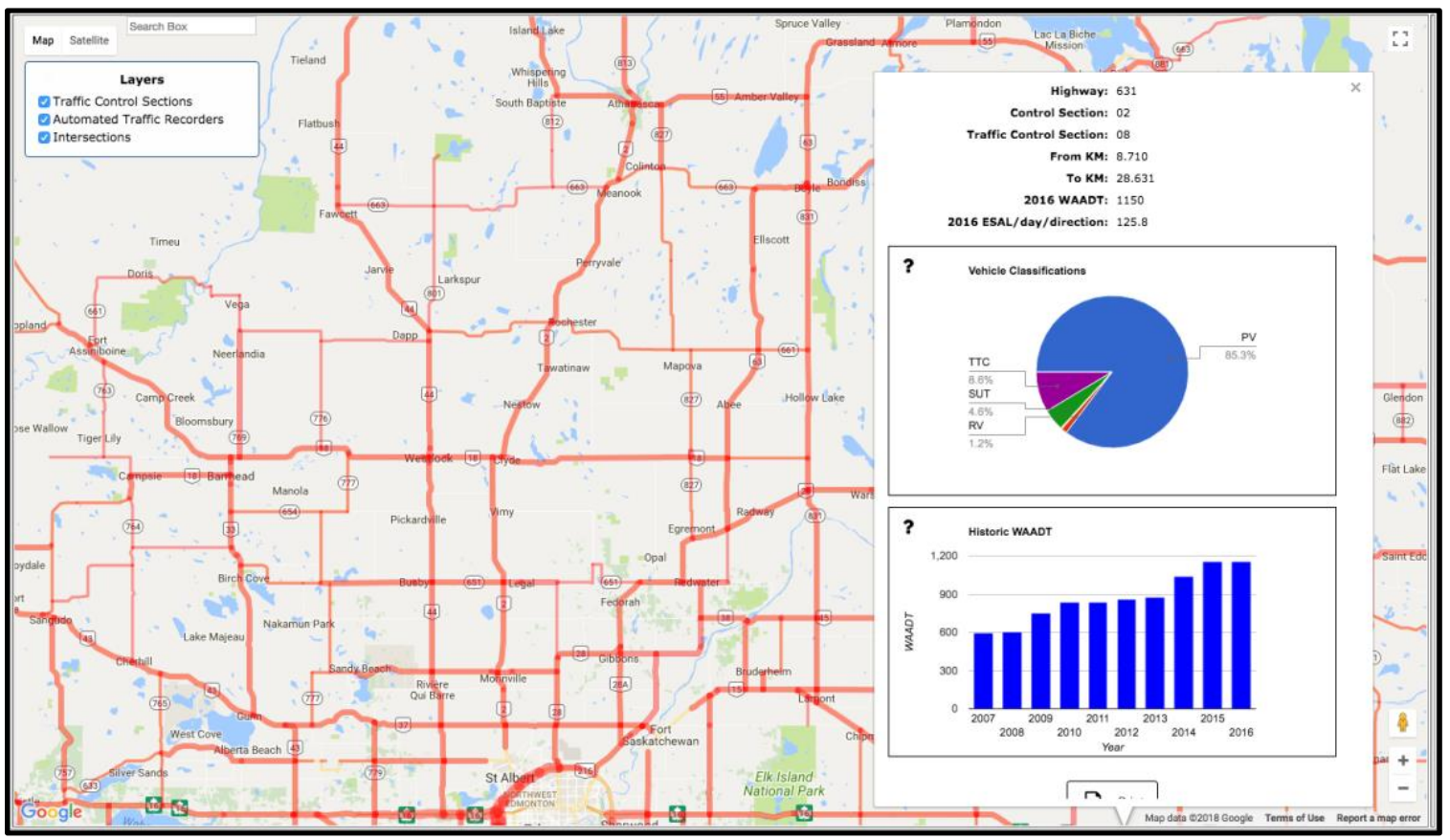

Figure 5: Alberta Ministry of Transportation traffic data dissemination tool (Alberta Ministry of Transportation, 2017)

\subsubsection{Saskatchewan}

The Government of Saskatchewan displays their traffic data in .pdf maps available on their website (Saskatchewan Ministry of Highways \& Infrastructure, 2017). Maps containing highway traffic volume data (AADT), the national highway system, and weight classification, as well as other transportation related maps, are available for download.

The highway traffic volume map displays the AADT of each highway segment, with continuous and short-term count estimations represented by different colours. The weight classification map assigns different colours to highway segments to represent weight class. 
Due to the static nature of the maps, the method of attributing volume to highway sections, including how the highway network is segmented, is unclear. Locations of count stations are also unknown.

\subsubsection{Manitoba}

Manitoba Highway Traffic Information System (MHTIS) GeoMedia WebMap is Manitoba Infrastructure's (MI) traffic data dissemination tool (Manitoba Highway Traffic Information System, 2017). The website gives options of viewing several maps: a count stations map, annual flow maps from years dating back to 2006, truck traffic flow maps for 2008 or 2013, and an intersection turning movement count map.

The count stations map displays the point locations of the numerous Permanent Count Stations (PCS), Coverage Count Stations (CCS), and Town Count stations across the province. Station points can be selected to reveal the station type, number, its location, as well as a link to traffic statistics from past years. The map also displays highways and highway regions, all of which are in layers that can be turned on and off.

Traffic flow maps, including truck traffic flow maps, display the magnitude of traffic volume as AADT on highways by the thickness of the highway sequence, where greater line thickness implies greater AADT. A highway sequence can be selected to reveal its attribute data, including the calculated AADT, ASDT, and $30^{\text {th }}$ highest hour statistics, as well as the sequence's length, number, and attributed station.

The intersection counts map displays the count locations as points on the highway network. Upon selecting an intersection count point, attribute data of the count location appear, including the intersection, years/dates the count(s) was completed, and links to traffic count summaries. The intersection count map has a highway layer and counts layer that can be turned on and off by the user. 
All maps have the ability to measure distance, area, and give coordinates of a particular location. The maps also have predefined queries to find specific towns, highway sequences, and counts near a particular town in the case of the count maps. Figure 6 gives an overview of the tool.

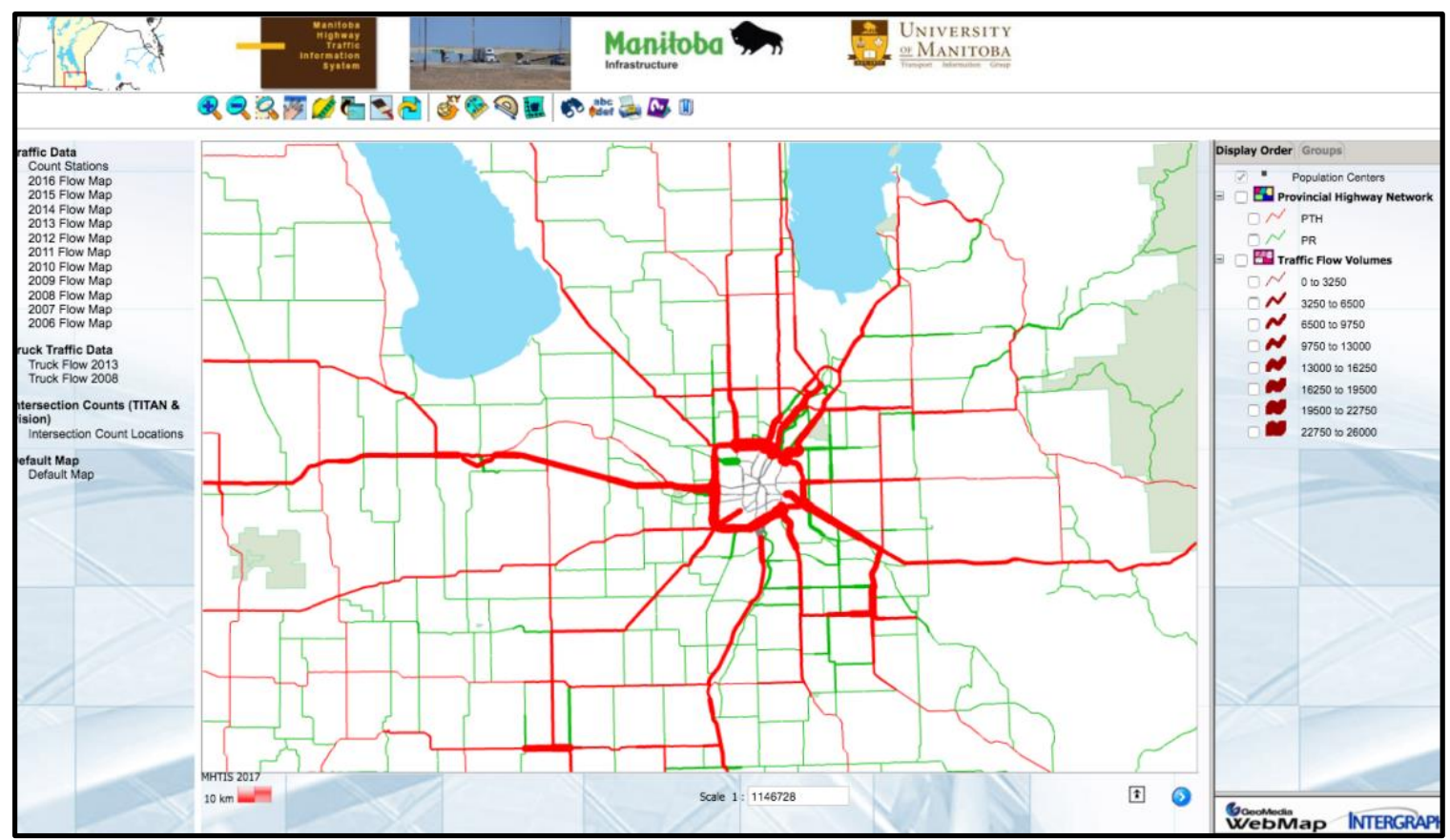

Figure 6: Manitoba's traffic data dissemination tool (Manitoba Highway Traffic Information System, 2017)

\subsubsection{Ontario}

Ontario's Ministry of Transportation utilizes a Google-based map tool for data dissemination (Ontario Ministry of Transportation, 2010). The tool allows access to traffic volume data such as AADT, AADT growth, AADTT and value of goods over past years, as well as highway level of service, congestion analysis, commercial vehicle traffic volumes, and equivalent single axle loads (ESALs) in additional maps. AADT is represented as red highway segment lines, where the magnitude of AADT is represented by the thickness of the lines. However, the design of the program does not allow the user to see the discrete highway segments. Highway segment start/end points can be inferred at locations where traffic volume substantially changes as there is a 
noticeable change in line thickness between the two highway segments; however, this method is not applicable at all locations, such as where there is little to no change in traffic. The user can turn data layers on and off to allow for viewing of different information. The tool also allows the user to search for location by coordinates.

Additional capabilities of the tool include measuring distance or area and allowing the user to mark up the map. The tool's base map can be changed or removed depending on user preference. While the user cannot download data from the map, traffic volume data can be downloaded in a PDF file elsewhere on the website (Ontario Ministry of Transportation, 2016). Figure 7 gives an overview of the tool.

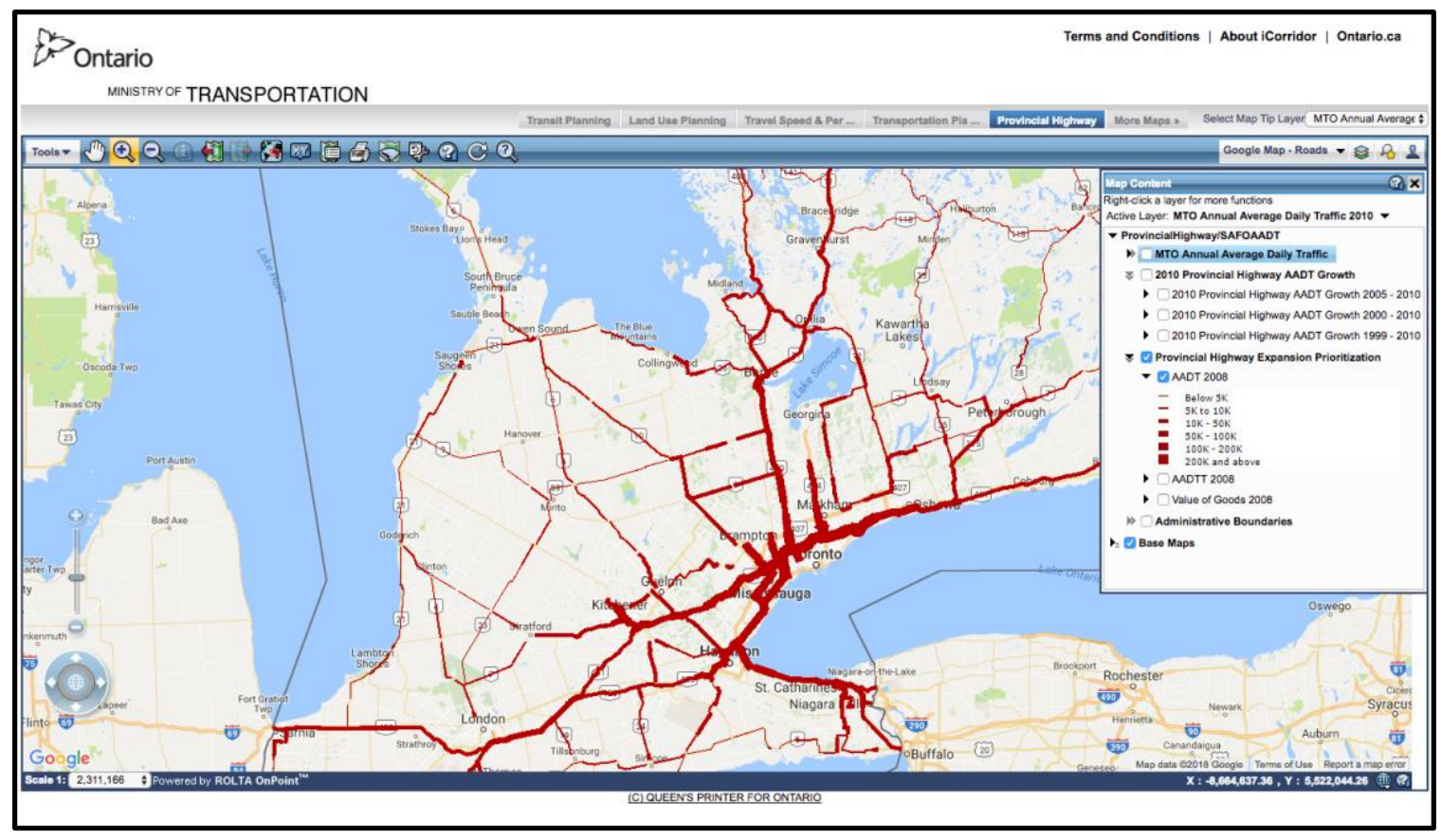

Figure 7: Ontario Ministry of Transportation iCorridor traffic data dissemination tool (Ontario Ministry of Transportation, 2010)

\subsubsection{Nova Scotia}

Nova Scotia has an online dataset in which it stores AADT and percent truck values of different highway sections throughout the province (Nova Scotia Transportation and Infrastructure Renewal Department, 2017). While the website offers the option to 
visualize the data in a map, the data have no location information (i.e., latitude/longitude coordinates) and therefore cannot be viewed in a map. The user can download data from this webpage in a number of different file formats, including CSV and XML.

\subsubsection{Minnesota}

The Minnesota Department of Transportation (MnDOT) has created an independent map-based traffic data dissemination tool (Minnesota Department of Transportation, 2017). This tool allows access to traffic data and statistics such as traffic count locations, WIM data, ATR data, and AADT. Traffic volume is represented on highway segments by a graduated colour scheme, and count locations are represented as points. Each data type exists in its own layer, allowing the user to choose which data are visible in the map; however, some layers are only visible at specific zoom levels. The "Identify" tool allows the user to select an element and view element specific information, such as location, technology type, etc., as well as data pertinent to the element, such as the traffic volume summaries associated with a particular ATR. The user can define queries by selecting the "Query" button, and predefined location-based queries can be accessed by selecting the "Jump to" button.

Highways are segmented into sequences that are assigned a sequence number; presently sequence numbers have values between 1 and 70549 . By visual inspection, sequence end points are not discernible except at locations of major traffic volume change where a colour change occurs. The highways are sequenced in such a way to allow for a 1:1 ratio of count sites to sequences. This means some sequences cross intersections and jurisdictional boundaries since there are not enough count sites to sequence the highway network at all intersections and boundaries. Figure 8 gives an example of this. Note the coloured segments represent different highway sequences and the point represents a count location. 


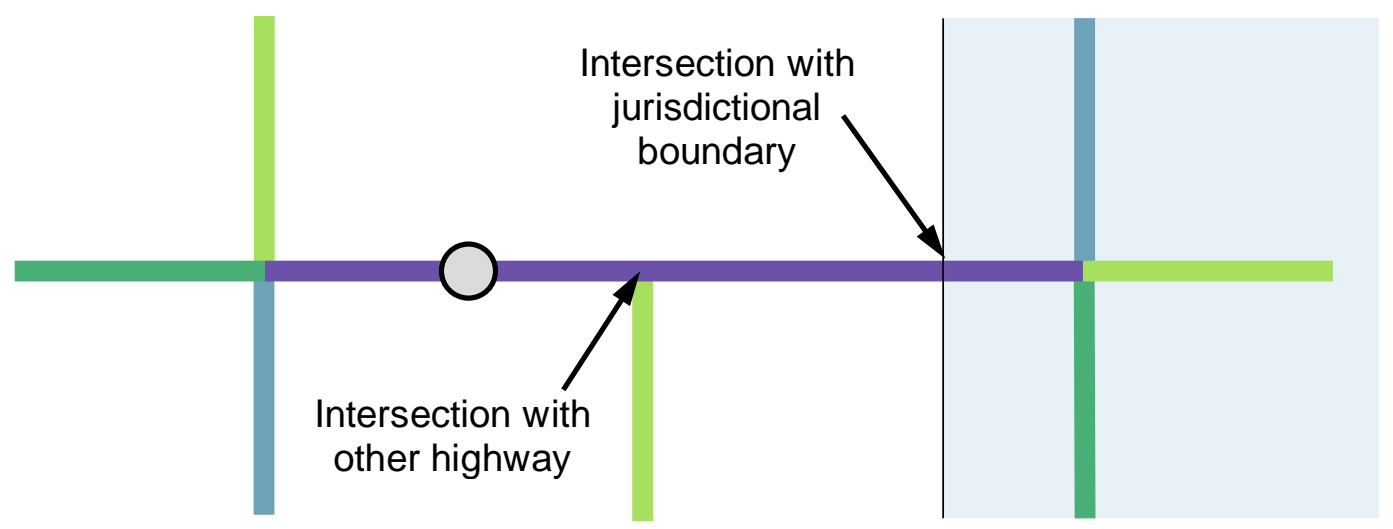

Figure 8: Example of sequencing highway network to create a 1:1 ratio of count sites to traffic sequences

As can be seen, the highway network is not sequenced at the centre intersection to allow for a 1:1 ratio of count sites to sequences. Were the segment sequences at this intersection, the segment to the right of the intersection would not have a count site, resulting in a 0:1 count site to sequence ratio.

Element-specific data are available when the element is selected with the "identify" tool; the user can download these data as a PDF. Larger volumes of traffic data such as ATR hourly volume reports, historical AADT tables, Vehicle Miles Travelled (VMT), etc., are available for download in a variety of file formats by selecting the "Get Data" button. 
Figure 9 gives an overview of the tool.

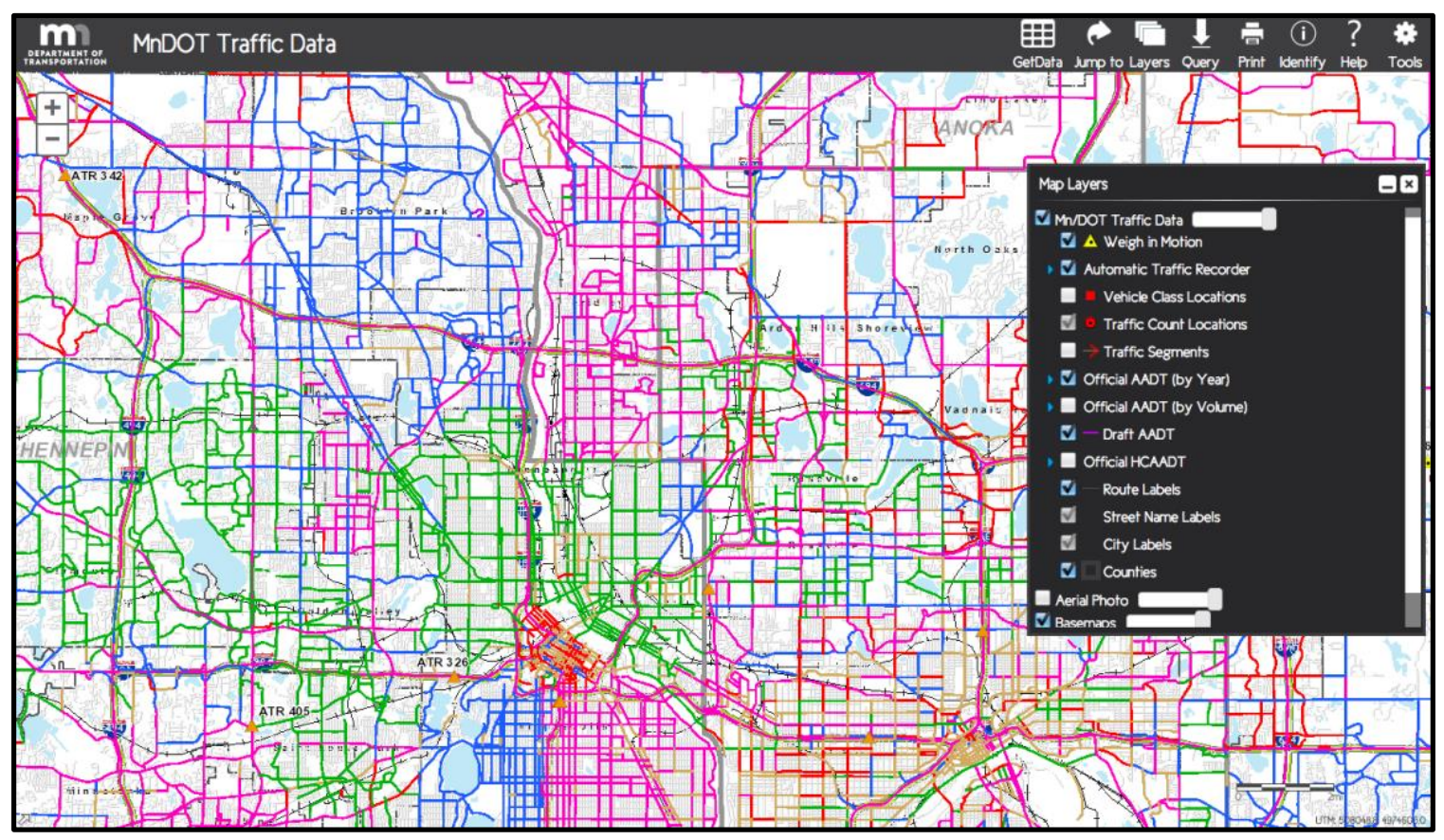

Figure 9: Minnesota Department of Transportation traffic data dissemination tool (Minnesota Department of Transportation, 2017)

\subsubsection{North Carolina}

North Carolina Department of Transportation (NCDOT) utilizes ESRI's ArcGIS suite to create a traffic data dissemination tool (North Carolina Department of Transportation, 2016). This tool allows access to traffic volume data (as AADT) on North Carolina's highway network, where the value of the AADT estimate of a highway segment is represented by the colour of the segment. The specific value can be obtained when the highway segment is selected, in addition to beginning and end mile post measurements of the segment, AADTT, and estimated AADT for single unit trucks (class 4-7, AADT_SU) and for multi-unit trucks (class 8-13, AADT_DU) (North Carolina Department of Transportation Traffic Survey Group, 2016).

Individual stations can also be selected, which display the station ID and information on past year AADTs, as well as more location related information such as the county name, 
route identifier, and a description of the station location (North Carolina Department of Transportation Traffic Survey Group, 2016). Station points are assigned colour based on the type of highway they are located on, such as Interstates, US Routes, NC Routes, Secondary Routes, and Non-System Routes.

The highway network is divided into segments with assigned RoutelDs. Some of these segments are further divided to assign traffic volume, but RoutelDs stay the same, so two segments can have the same route number but different AADTs. It appears segments are divided to create a 1:1 ratio of count stations to traffic segments, which means some segments may cross intersections or jurisdictional boundaries similar to Minnesota.

Additional capabilities of the map include the option to turn on/off layers, search addresses and locations in a search bar, view attribute tables of the different layers, and measure area, distance, or find the latitude/longitude pair of a point location using the measurement tool. Data in the map are available for download, including the AADT and vehicle classification station shapefiles, and the traffic segment shapefile. Public spatial data available through ArcGIS Online can be added to the map. Figure 10 gives an overview of the tool. 


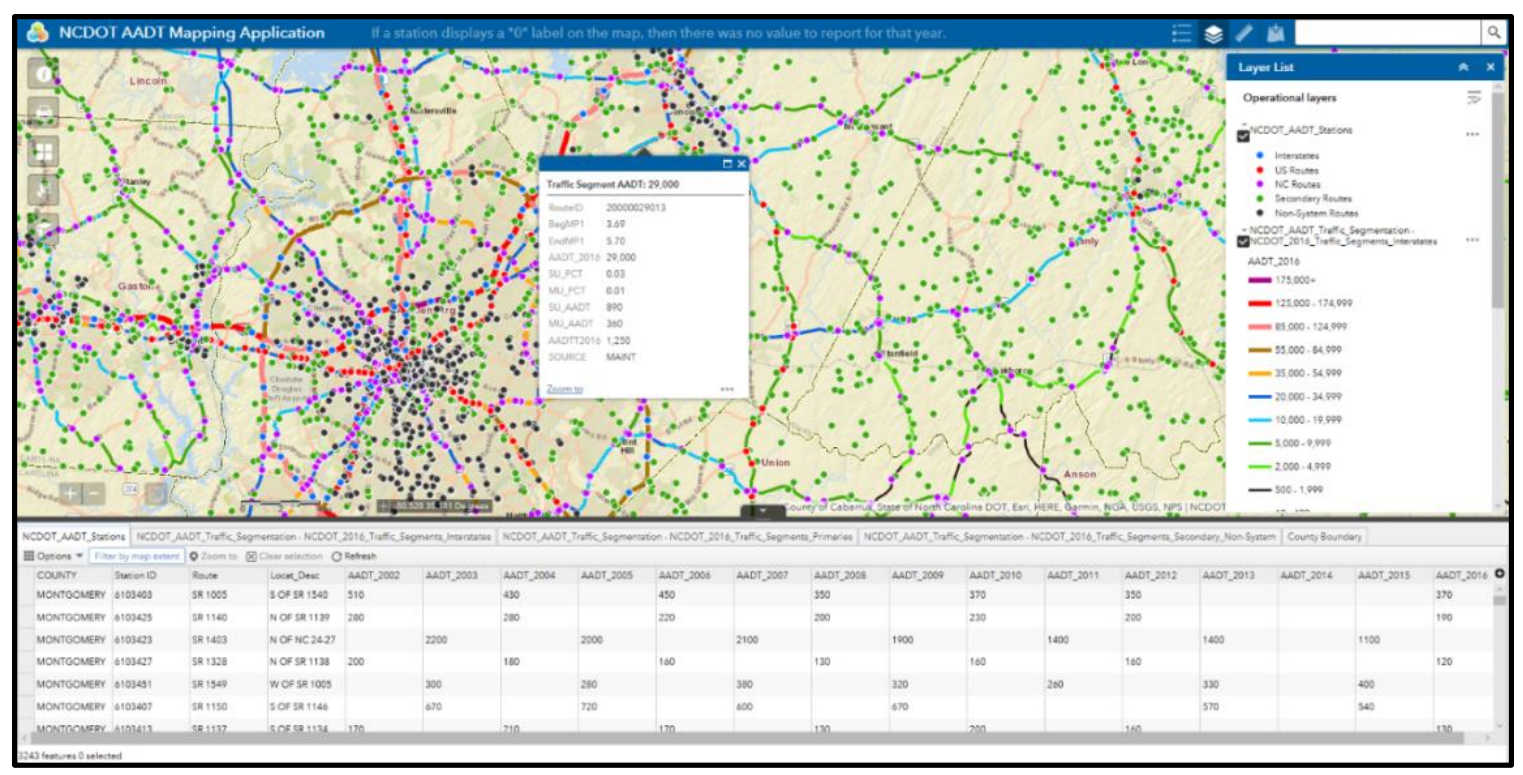

Figure 10: North Carolina Department of Transportation traffic data dissemination tool (North Carolina Department of Transportation, 2016)

\subsubsection{Colorado}

The Colorado Department of Transportation (CDOT) utilizes ESRI's ArcGIS suite to create a traffic data dissemination tool (Colorado Department of Transportation, 2017). This tool shows traffic volume by colour on highway segments, with approximated AADT displayed as a number on the segment. AADT values in black print are estimated from a short duration count, or coverage count station, whereas AADT values in red writing are calculated from a continuous traffic recorder, or permanent count station.

Highways are divided into segments called "routes", which are further divided into smaller segments to assign traffic volume. As a result, two segments can have the same route number but different AADTs. Since features cannot be selected, it is difficult to discern route start and end points, and similarly, difficult to determine how traffic data are attributed.

Attribute tables of the map layers, which are short duration counts, continuous traffic recorders, AADT labels, and highway labels, are located in the tab at the bottom of the 
screen. While the layers on the map are not selectable, the attribute tables update when zooming in and out. The attribute tables can also be filtered with basic expressions, such as "AADT is greater than 30000 ". This filter applies to the table, however not the map. Specific addresses and locations can also be searched using the search bar at the top of the map. Figure 11 gives an overview of the tool.

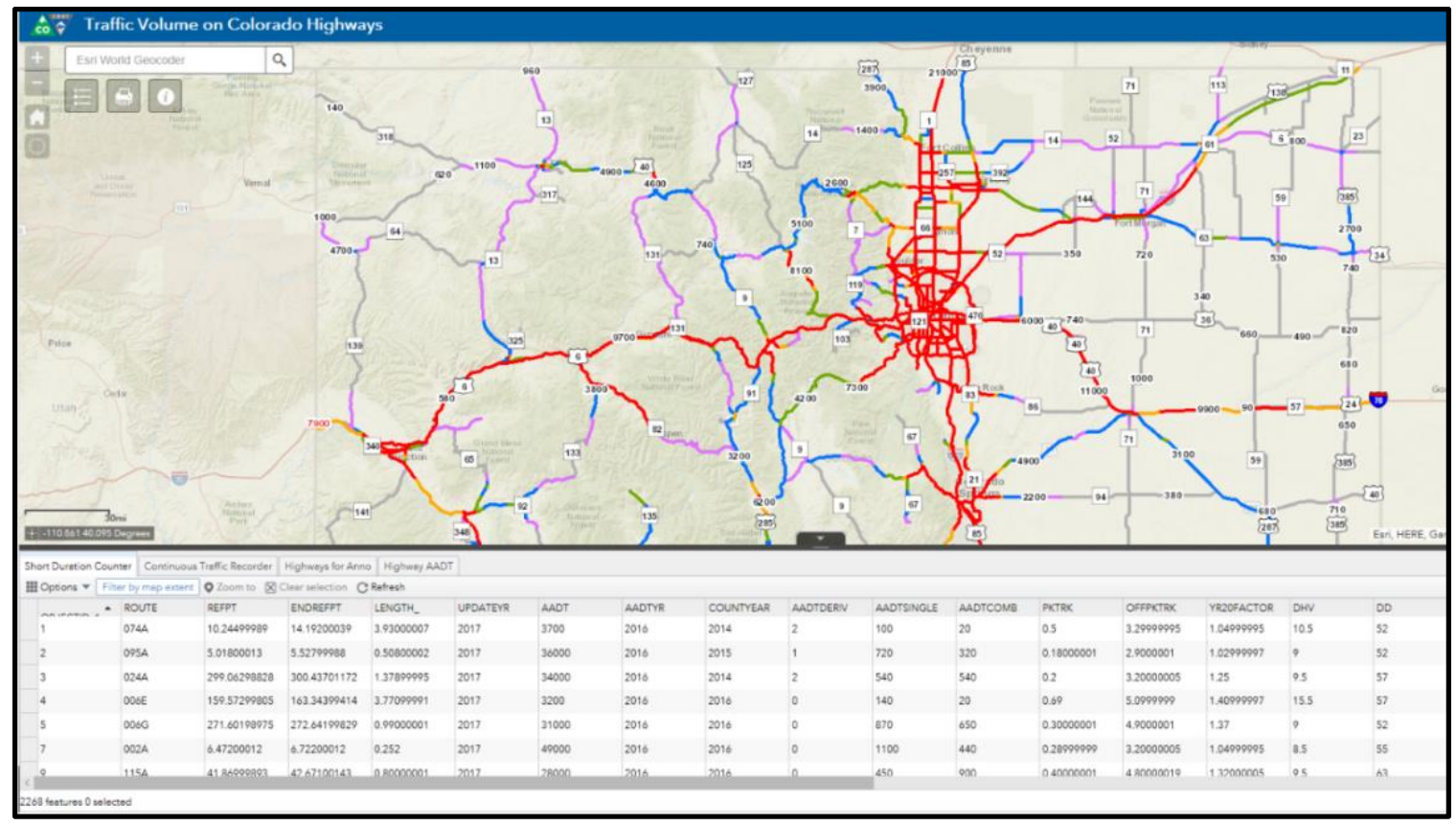

Figure 11: Colorado Department of Transportation traffic volume data dissemination tool (Colorado Department of Transportation, 2017)

\subsection{SUMMARY OF NORTH AMERICAN TRAFFIC DATA DISSEMINATION TOOLS}

Table 1 summarizes the capabilities of each of the data dissemination tools discussed in sections 2.3.2 through 2.3.9. The various tools are summarized based on three categories: the data that are accessible and/or visible in the tool, how the highway network is sequenced for traffic volume, and how data are attributed to the sequenced highway network. In the data accessible/visible category, there are three types of data that are of interest: stations, traffic segments, and traffic volume. For stations, a check mark is given if the tool displays stations and their data, and an " $X$ " mark if it does not. 
For traffic segments, a check mark is given if the tool shows discrete traffic segments, including their start and end points, and an "X" mark if the tool does not display individual traffic segments. The traffic volume data are given a check mark if the tool visualizes and allows access to traffic volume data, a hyphen ("-") if the tool does one of the two, and an " $X$ " mark if it does neither. 
Table 1: Summary of publicly available traffic data management tools in North America

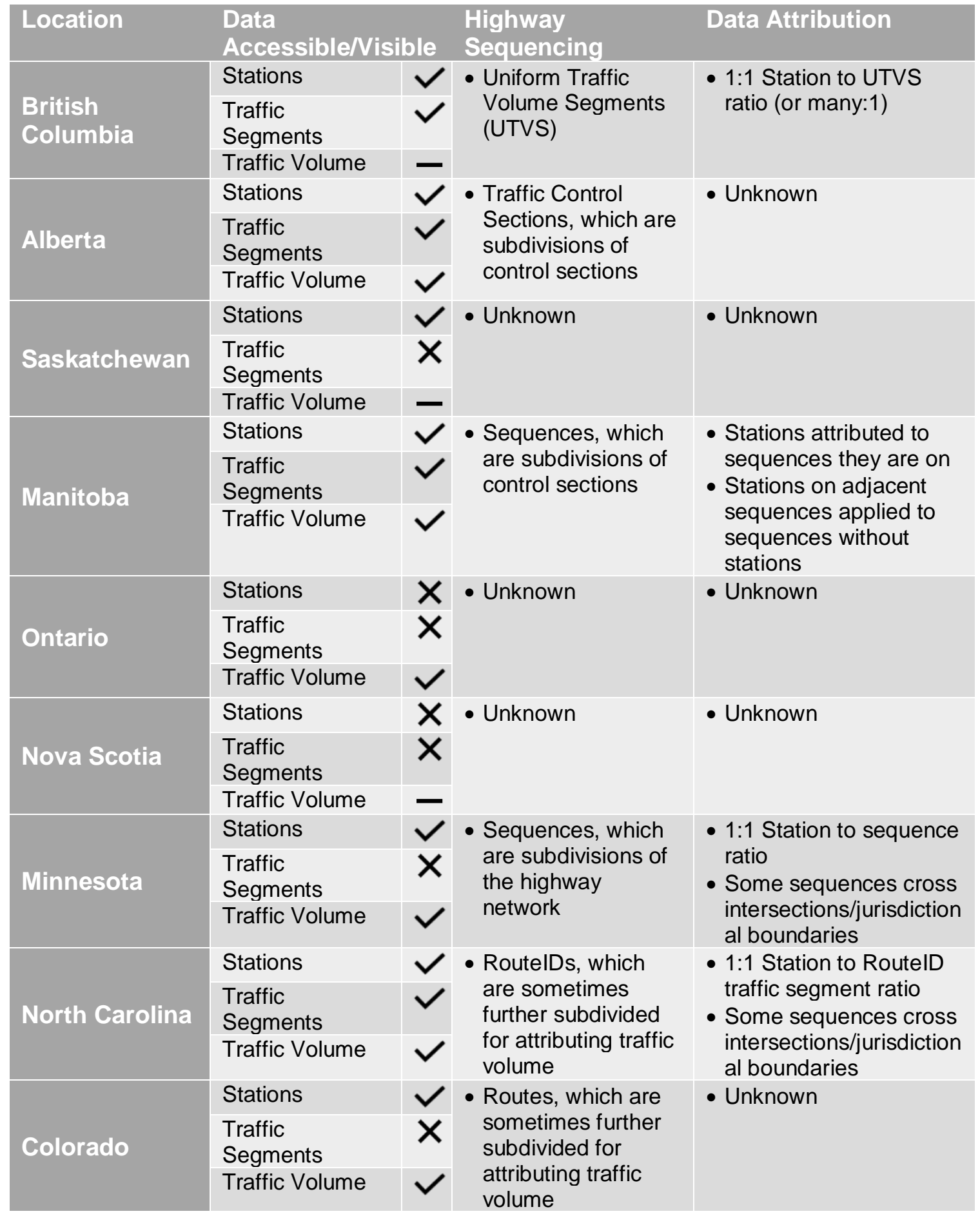

Note: "Unknown" means that the information was not publicly available via the online dissemination tool. 
As can be seen, Alberta, Manitoba, and North Carolina are the only locations that visualize and allow access to all three types of key data. Nova Scotia, only having the ability to view data but not visualize them, is given an " $X$ " in both Stations and Traffic Segments, and a "-" for traffic volume data. All other locations contain some combination of the three types of data.

It can also be noted that while some methods of highway sequencing and data attribution are unknown, some appear to have similarities, such as Minnesota, North Carolina, and British Columbia. This finding indicates the need for the research undertaken in this thesis. 


\section{METHODOLOGY}

This chapter describes the research methodology, which involves two main procedures: sequencing the highway network and attributing traffic data to the sequenced highway network. The sequencing procedure involves segmenting the highway network following the assumption of homogeneous traffic volume. The attribution procedure involves attributing sites and their traffic volume characteristics to the highway network sequences. The methodology is an iterative process in that the network may require additional sequencing upon first completion of the attribution procedure. After attribution is an evaluation procedure in which the segments that did not abide by any of the attribution rules are considered for additional sequencing, followed by attribution of count sites to sequences. Figure 12 schematically depicts the methodology for this research.

\section{Sequencing}

- Determine locations of traffic volume change

- Split network into sequences at locations of traffic volume change

- Numerate sequences
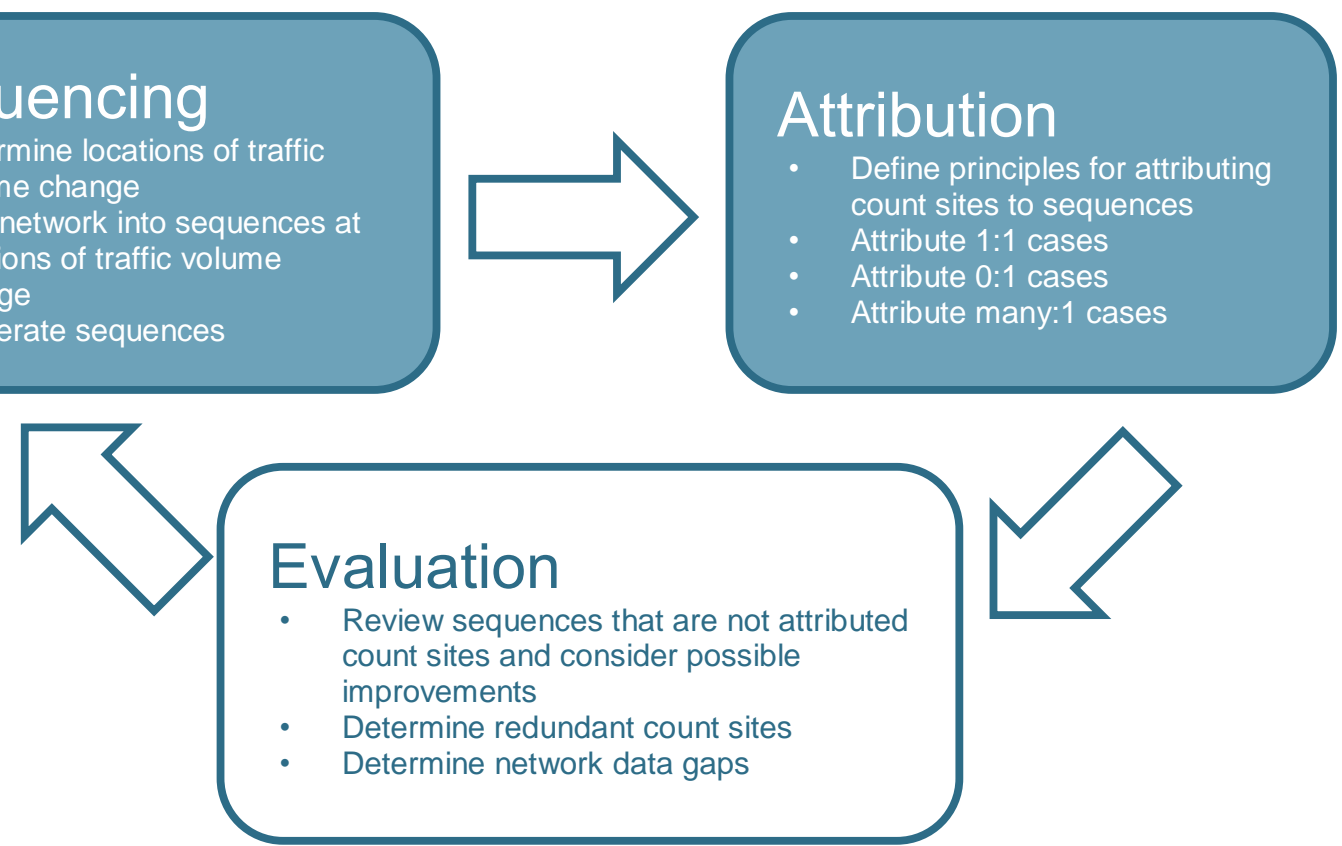

Figure 12: Schematic depiction of the sequencing, attribution, and evaluation procedures applied in this research. 
MI's LRS provides the analytical foundation for the sequencing and attribution procedures. The chapter begins by describing the data structure of the LRS before outlining the sequencing and attribution procedures.

\subsection{DATA STRUCTURE}

\subsubsection{Spatial Data}

Manitoba's highway network comprises Provincial Trunk Highways (PTHs), Provincial Roads (PRs), and 600 series highways. Highways 1 to 190 are considered PTHs, highways 200 to 596 are considered PRs, and highways in the 600s are 600 series highways. 600 series highways include access roads, earth roads, and "other" roads. Table 2 gives the total single-centreline lengths of the three types of highways and Figure 13 shows a map of Manitoba's provincial highway network.

Table 2: Total single-centreline lengths of the three highway types

\begin{tabular}{|l|l|}
\hline \multicolumn{1}{|c|}{ Highway Type } & Length (km) \\
\hline Provincial Trunk Highway & 7461 \\
\hline Provincial Road & 10325 \\
\hline 600 Series & 484 \\
\hline
\end{tabular}




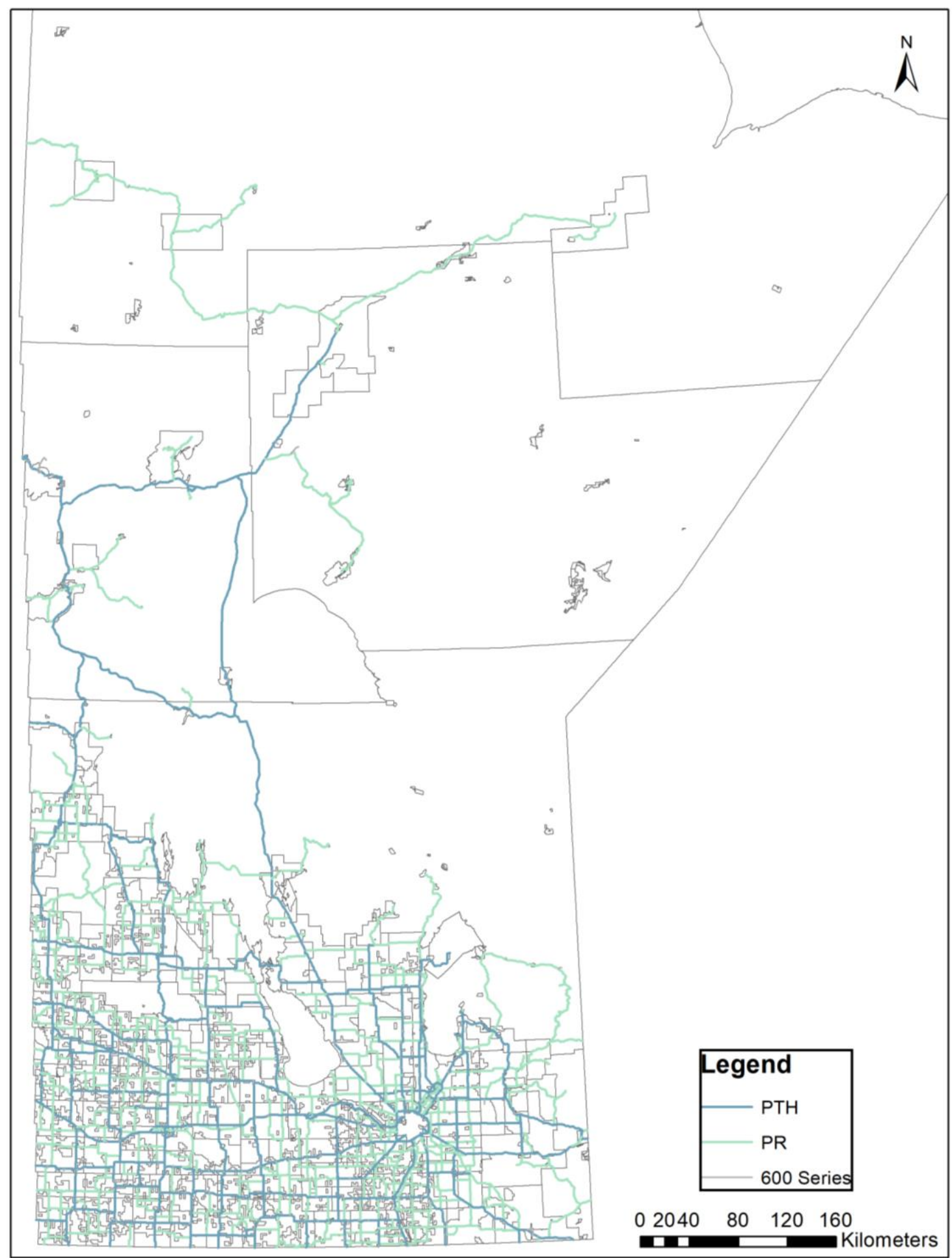

Figure 13: Manitoba's Provincial Trunk Highways, Provincial Roads, and 600 Series Roads MI's spatial file is a double-centreline representation of Manitoba's highway network. This means that divided highways are represented as two lines along the centre of the 
highway for each direction of flow, and undivided highways are represented by a single line along the centre of the highway. Figure 14 illustrates these concepts.

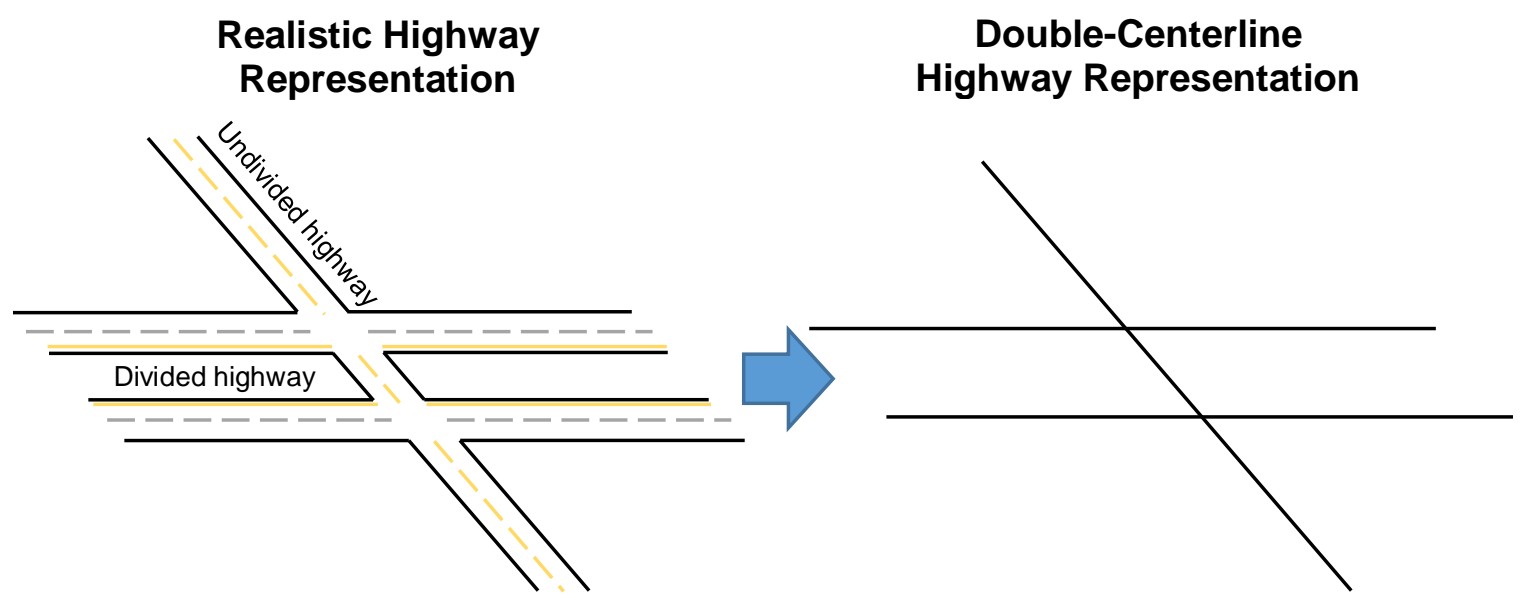

Figure 14: Ml's double-centreline representation of the Manitoba highway network MI has divided Manitoba's highways, into segments called traffic control sections, or sections, for the purpose of spatial referencing. Section break points occur at:

- all intersections with PTHs (i.e., PTH/PTH, PTH/PR, and PTH/600 Series intersections);

- locations where a highway changes from multi-lane to undivided, or undivided to multi-lane;

- intersections with local/municipal roads; and

- bridges.

Figure 15 and Figure 16 demonstrate the concept of control sections. Sections vary in length from a few hundred metres to nearly $80 \mathrm{~km}$. Table 3 summarizes the sections based on eight length bins, excluding 600 Series sections. 


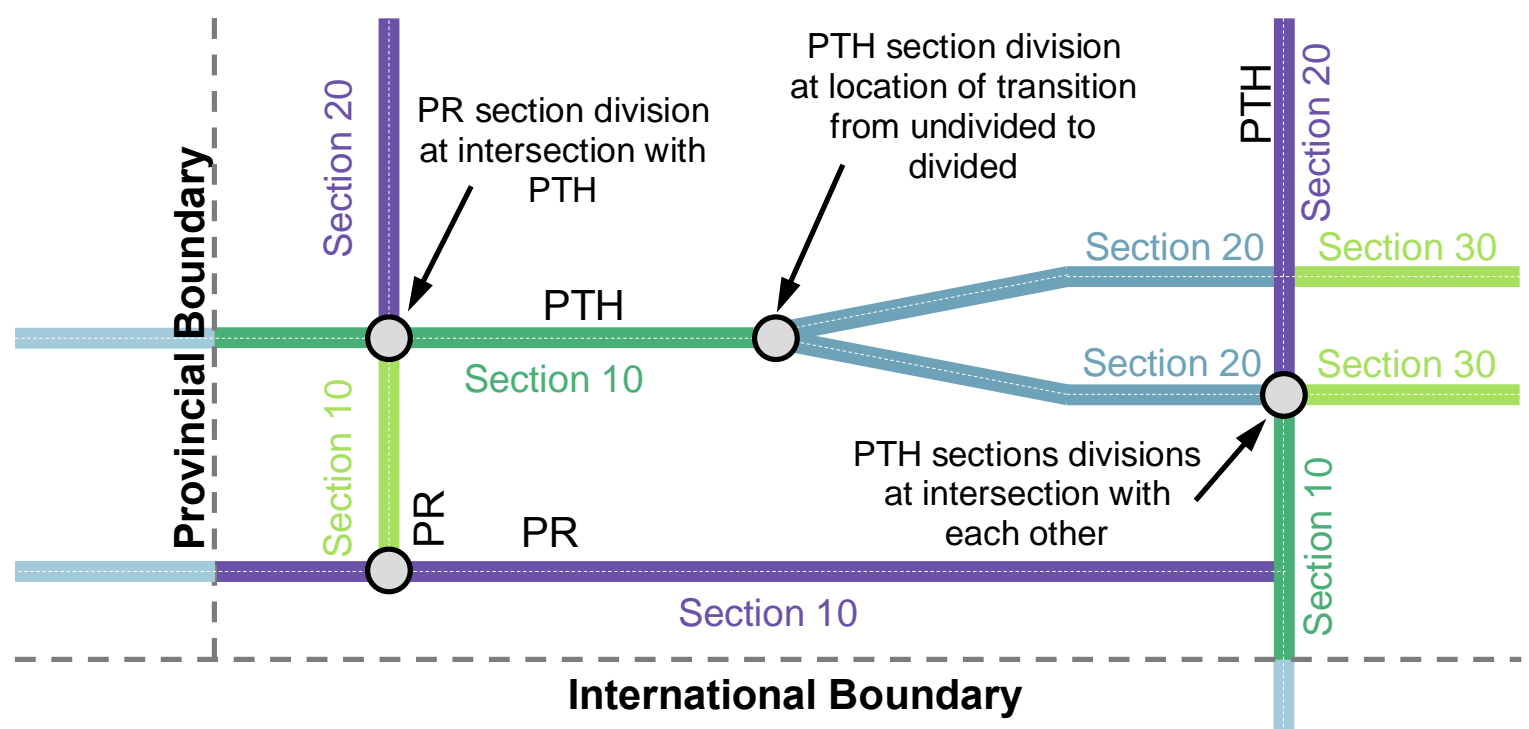

Figure 15: Example of traffic control sections defined by MI

Table 3: Summary of sections by length

\begin{tabular}{|l|l|l|l|}
\hline Section Lengths $\mathbf{( k m )}$ & Section Count & Total Length $\mathbf{( k m )}$ & $\%$ of Network \\
\hline $0-9.99$ & 339 & 1697.20 & 9.54 \\
\hline $10-19.99$ & 366 & 5590.44 & 31.43 \\
\hline $20-29.99$ & 256 & 6157.86 & 34.62 \\
\hline $30-39.99$ & 77 & 2588.84 & 14.56 \\
\hline $40-49.99$ & 27 & 1170.97 & 6.58 \\
\hline $50-59.99$ & 8 & 435.88 & 2.45 \\
\hline $60-69.99$ & 1 & 67.20 & 0.38 \\
\hline $70-79.99$ & 1 & 77.60 & 0.44 \\
\hline Total & 1075 & 17785.98 & 100 \\
\hline
\end{tabular}




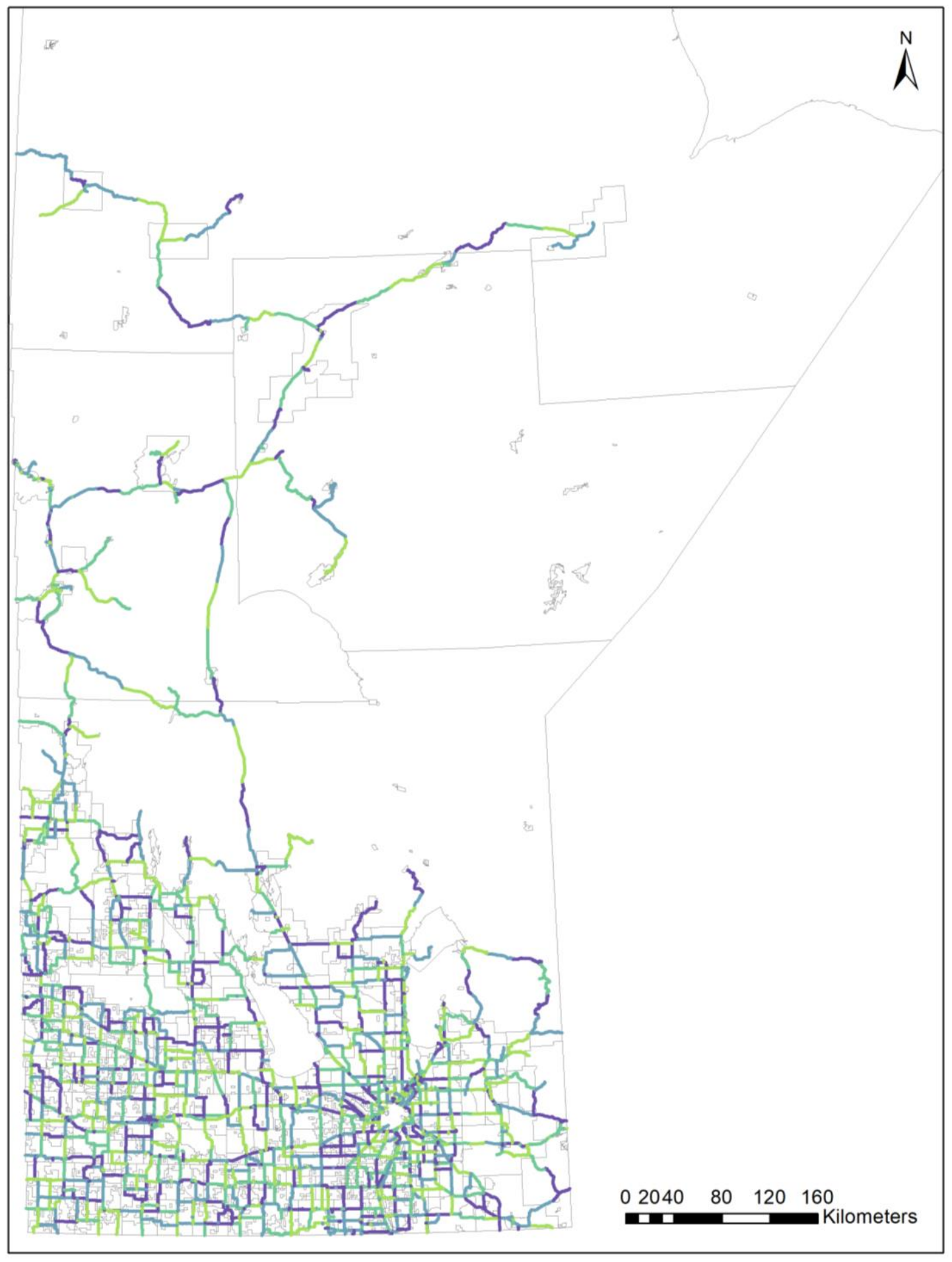

Figure 16: Manitoba highway sections 
Section topology proceeds west to east and south to north, with section numbers increasing in the easterly and northerly directions. Each highway segment has its own row in the database that contains the segment's attribute data, including highway number, highway region, section number, start and end kilometre, length, and a brief location description. Each highway section is assigned a control section key (CS_KEY), which is created using the two-digit highway region, three-digit highway number, threedigit section number, one-letter road type and one-letter road direction. The three road types in the MI's LRS are:

- $\quad$ highways $(\mathrm{H})$, which include PTHs, PRs, and Access Roads (PAs);

- $\quad$ earth roads $(\mathrm{E})$; and

- other roads $(\mathrm{O})$, which include federal, municipal, provincial or private jurisdiction roads that are necessary for continuity of the network (Highway Planning \& Design Branch, 2005).

The three letters representing road direction are A, B and U. A represents the ahead direction of a divided highway, which is typically the right-hand roadway when travelling east or north along a highway. B represents the back direction, which is the left-hand roadway when travelling east or north along a road. $U$ represents an undivided road. Figure 17 illustrates the CS_KEY numeration.

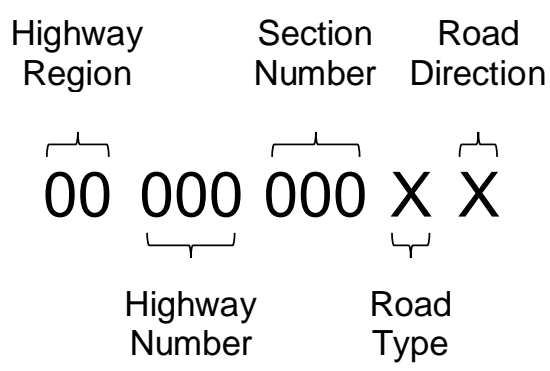

Figure 17: Illustration of Control Section numeration 
The highway sections as defined by MI often cross one or more intersections (see Figure 15); PRs are split at PTH intersections, however this does not mean PTHs are also split at these locations. This is problematic for the management and dissemination of traffic data because traffic volume cannot be assumed homogeneous along a section. For example, traffic volume changes at intersections, and so applying a single traffic volume to a section that crosses an intersection does not facilitate an accurate representation of traffic volume.

Consequently, to properly manage and disseminate traffic volume data, there is a need to subdivide sections into shorter sequences, for which the homogeneity assumption is more realistic. One or more sequences comprise a section, and one or more sections comprise a highway. Figure 18 demonstrates this concept.

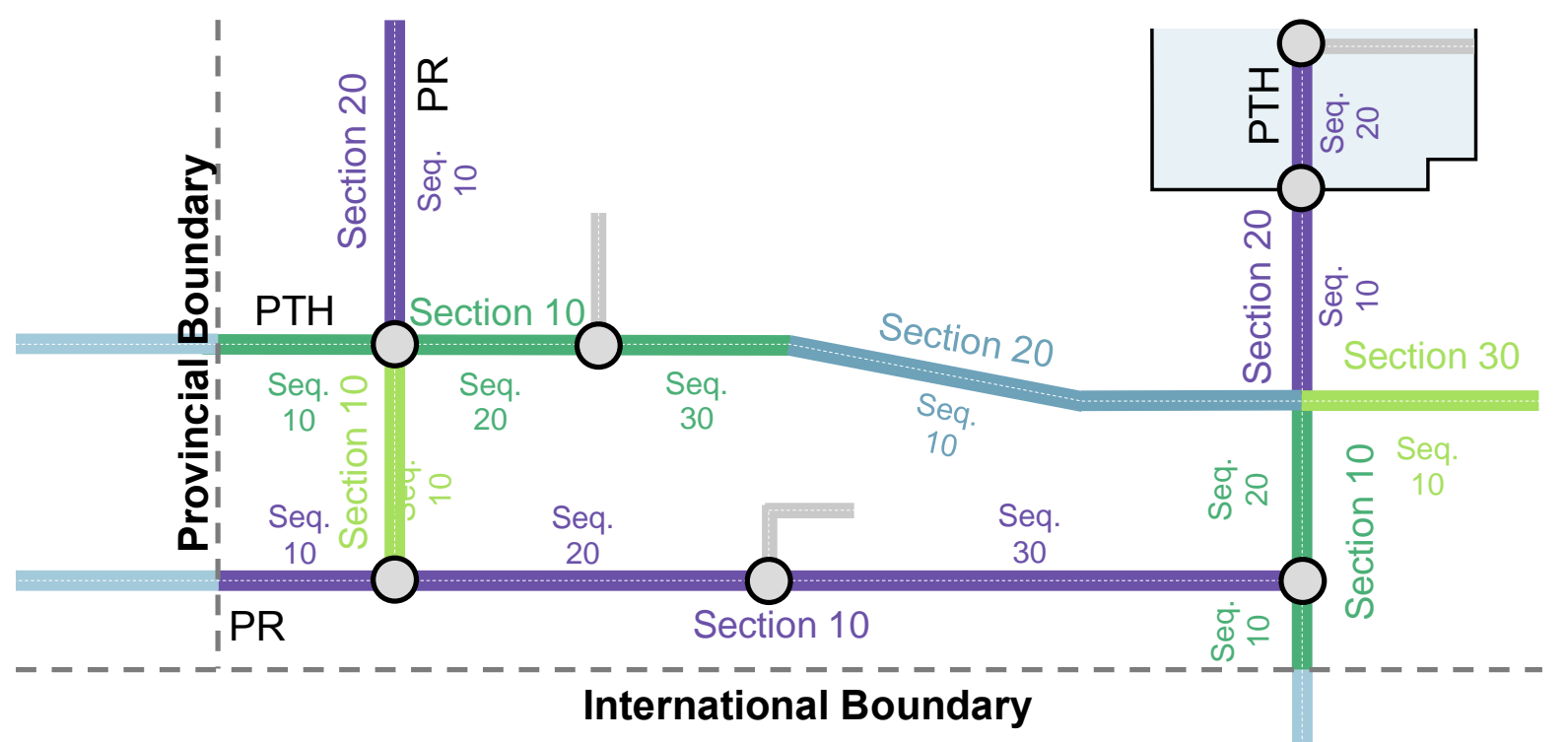

Figure 18: Example of highway sections further divided into highway sequences to allow for the homogeneity assumption 
Sequencing allows for traffic volume data to be attributed to Manitoba's highways, but must be done so logically to maintain accuracy and user operability. The sequencing topology follows that of sections, with numeration increasing in the easterly and northerly directions. Criteria are applied to sequence the network consistently, and ArcGIS is utilized to apply these rules in sequencing the network. Section 3.2 provides further details.

\subsubsection{Traffic Count Sites}

MI collects traffic data on the Manitoba highway network using continuous and shortduration counts at 1770 locations. There are three types of continuous count sites, which are also known as Automated Traffic Recorders (ATR).

- Permanent Count Stations (PCS): equipment permanently embedded in the pavement that counts the number of passing vehicles.

- Automatic Vehicle Classifiers (AVC): equipment permanently embedded in the pavement that counts and classifies passing vehicles using a 15-vehicle classification scheme (see Appendix A). They also collect speed and axle spacing data.

- Weigh-in-Motion (WIM): equipment permanently embedded in the pavement that counts, classifies, and collects axle weight data of vehicles that pass the site. They also collect speed and axle spacing data.

There are a total of 85 continuous count sites located across the province.

Short-duration count sites are also referred to as Coverage Count Stations (CCSs). Equipment deployed at these sites collects vehicle or axle count samples (normally for a duration of 48 hours). There are two types of stations, including:

- loop stations, which utilize an inductive loop to detect passing vehicles, and 
- tube stations, which utilize pneumatic tubes to count vehicle axles.

There are 1685 short-duration counts in Manitoba. Each site is sampled once every three years. In the sampling year, two 48-hour counts are taken, typically during snowfree months.

Figure 19 displays them the 85 continuous count sites and 1685 short-duration count sites in a map. 


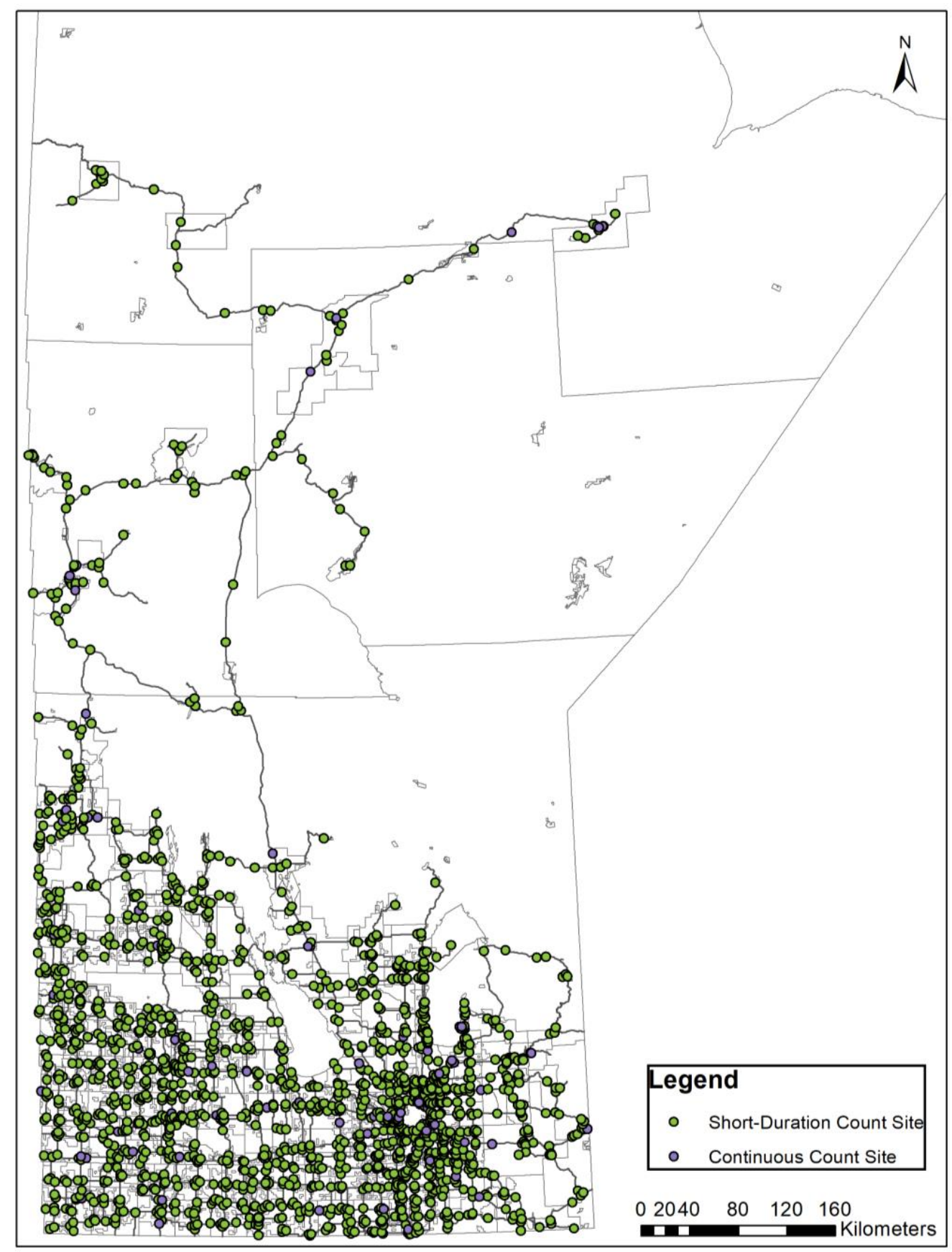

Figure 19: Continuous and short-duration count sites in Manitoba

\subsubsection{Data Limitations}

The limitations of the data follow: 
- The traffic data used are assumed to be of good quality; therefore, data validation was not a component of this research.

- The traffic data used in this research are collected for non-research purposes, rather for use in industry. Data collection is driven by data needs, and network coverage is not always the highest priority in data collection. Therefore, the network coverage is widespread but can be sparse in some areas while dense in others, introducing real-life data coverage limitations and the associated difficulties.

- The data are limited to what is available; consequently, if insufficient traffic data are collected, it cannot be used and is not re-collected.

- In Manitoba, short-duration count sites are surveyed once every three years, which introduces time gaps into the data. Further, the data collected at shortduration count sites are not necessarily collected in the same month of every collection year, introducing variability into the data. The data variability related to short-duration counts can result in significantly different traffic volumes collected from consecutive survey years.

- This research uses the 2015 LRS, which is the most recently available LRS at the start of this research. It is likely that the highway network has changed since 2015, and the most recent LRS would reflect these changes.

\subsection{SEQUENCING PROCEDURE}

This section outlines the procedure developed to sequence the highway network based on the homogeneity assumption. The "homogeneity assumption" is a pragmatic simplification that disregards immaterial changes in traffic volume along a highway. Its application underpins the development of criteria used in the sequencing procedure, which in turn facilitates the attribution of count sites to sequences. Locations of traffic 
generators, PTH/PR intersections, municipal road intersections, and urban boundaries must be considered when sequencing the highway network. Moreover, to create a continuous highway network: (1) sequences must touch end to end with no overlap, and (2) the beginning of the first sequence and the end of the last sequence must coincide with the section start and end points, respectively.

\subsubsection{Sequencing criteria derived from the homogeneity assumption}

The research derives four sequencing criteria from the homogeneity assumption.

- Criterion 1: Sections shall be subdivided into sequences at all PTH/PR intersections, so that no sequence shall cross a PTH/PR intersection. Intersections are locations where vehicles may enter or exit a given highway segment, and could therefore cause variation in traffic volume on either side of the intersection. For this purpose, sequences shall not cross PTH/PR intersections. This includes PTH/PTH, PTH/PR, and PR/PR intersections.

- Criterion 2: Sections shall be subdivided into sequences at intersections with 600 series highways. Similar to PTH/PR intersections, 600 series highway intersections introduce a location where vehicles may enter or exit a highway segment, causing changes in traffic volume. As a result, highway sequences shall not cross 600 series highways, including intersections of two 600 series highways. Currently, traffic volume on 600 series highways is not monitored. This sequencing criterion is included for the benefit of future changes to the highway network and volume characteristics.

- Criterion 3: Sections shall be subdivided into sequences at intersections with major municipal roads. Sections shall be split into sequences at intersections with major municipal roads. Some municipal roads intersect with provincial highways and cause significant traffic fluctuations, such as Kenaston 
Boulevard in Winnipeg that intersects with PTH 100. As a result, highway sequences shall not cross major municipal roads.

\section{- Criterion 4: Sections shall be subdivided into sequences at urban}

boundaries. Urban centres are another location of traffic fluctuation. Urban centres have a concentration of traffic sources and sinks, such as stores, gas stations, etc. There are several locations in the province where PTHs and PRs pass through urban centres, such as Brandon or Steinbach; however, traffic volume fluctuates throughout the urban centre. Therefore, sections shall be reasonably split into sequences at intersections with urban boundaries. Jurisdictions with populations of 5000 or greater are considered for this criterion, as this threshold encompasses all jurisdictions labeled as cities in Manitoba according to Statistics Canada (Statistics Canada, 2018). As stated in the introduction, this research does not consider traffic through urban centres. Urban centres have dense street networks, multiple access points, and interrupted traffic flow, among other complications, that make traffic monitoring complex (Rempel, Regehr, \& Montufar, 2013).

\subsubsection{GIS Functions}

The ArcGIS suite of tools is used to modify the spatial file of the Manitoba highway network (LRS_HIGHWAY_NETWORK_2015). Additional spatial files, including one of station locations (Station_Locations) and one of Manitoba subdivision boundaries (Manitoba_Subdivisions), are used for reference in modifying the LRS_HIGHWAY_NETWORK_2015. Appendix B contains the metadata of these files. Figure 20 outlines the steps of the sequencing procedure. 


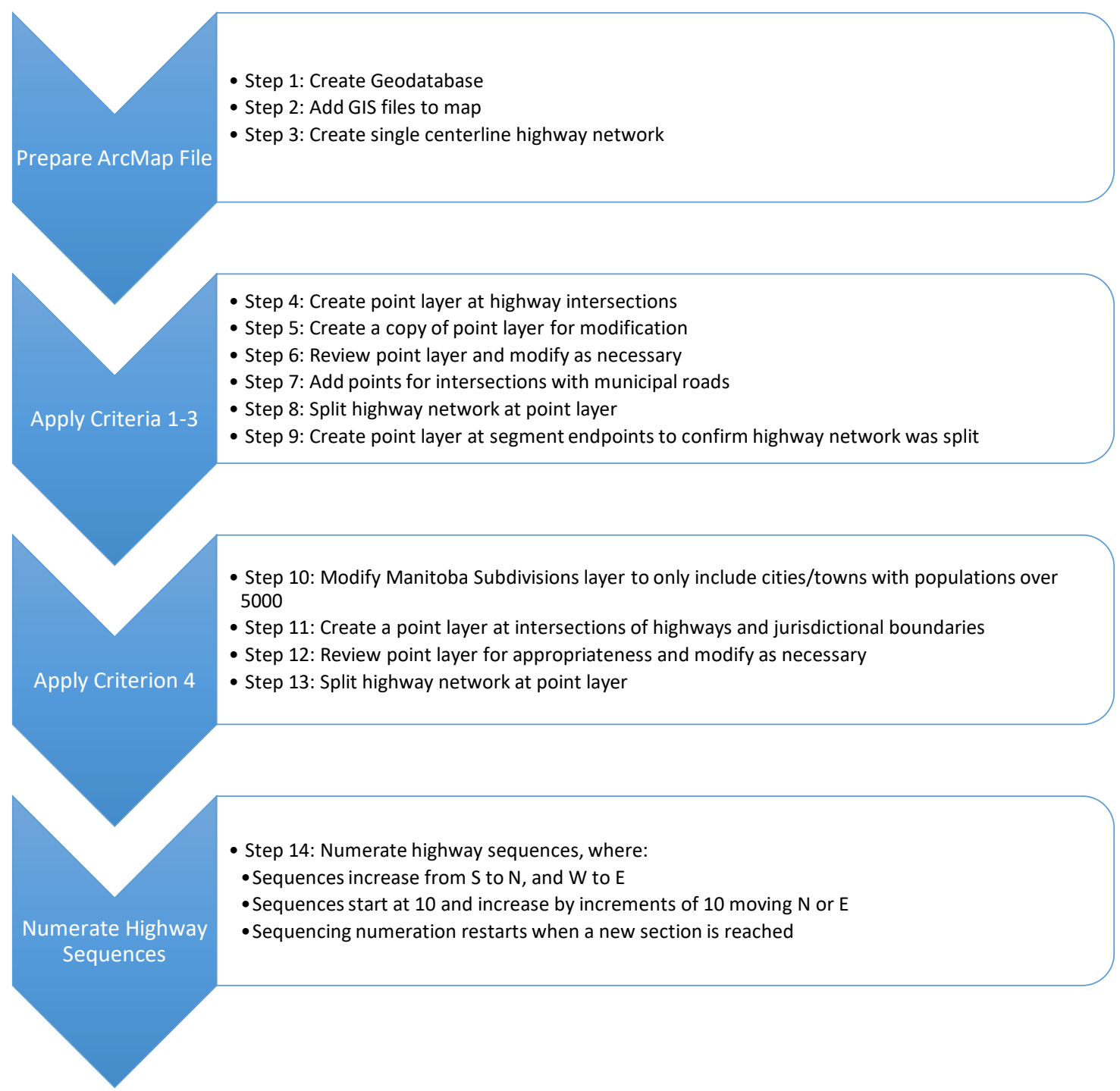

Figure 20: Flowchart of sequencing procedure

The more detailed steps taken to apply the criteria outlined in Section 3.2.1 and sequence the 2015 highways spatial file follow:

1. Step 1 creates a file geodatabase. A geodatabase is the original data structure of ArcGIS, and is therefore ideal when using ArcMap as it allows for more efficient use of ArcGIS tools and functions. For example, geometry fields, such as highway length, will automatically recalculate when using a geodatabase where as a shapefile would require the user to recalculate the geometry. 
2. Step 2 adds the key GIS files to the map. These files include the line-based MI 2015 highway network spatial file (LRS_HIGHWAY_NETWORK_2015), point-based count station location spatial file (Station_Locations), and polygon jurisdictional boundary spatial file (Manitoba_Subdivisions).

3. Step 3 creates a single-centreline highway network from the LRS_HIGHWAY_NETWORK_2015 file. Manitoba highways are assigned one of three directions: A, which represents the eastbound and northbound lanes; $B$, which represents the westbound and southbound lanes; and $U$, which represents an undivided highway. For simplicity in applying traffic volume data, the $A$ and $U$ directions are used to represent the network and combined directional volume is assigned to this single-centreline network, A_U_LRS_2015. Figure 21 illustrates the single-centreline representation of the highway network.

Double-Centerline Highway Representation

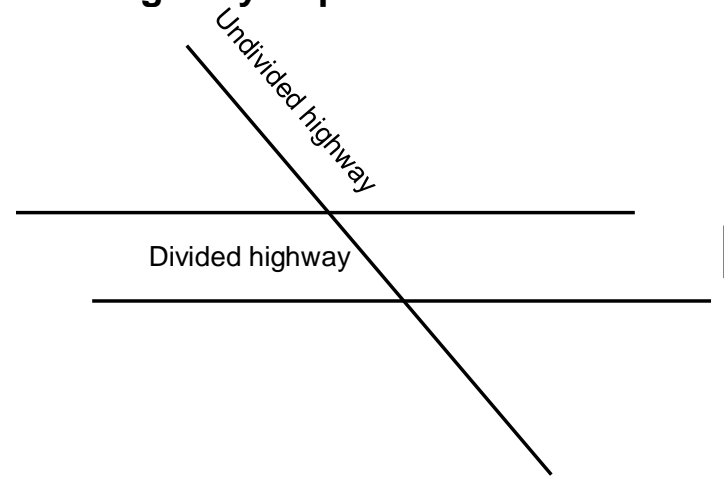

Single-Centerline Highway Representation

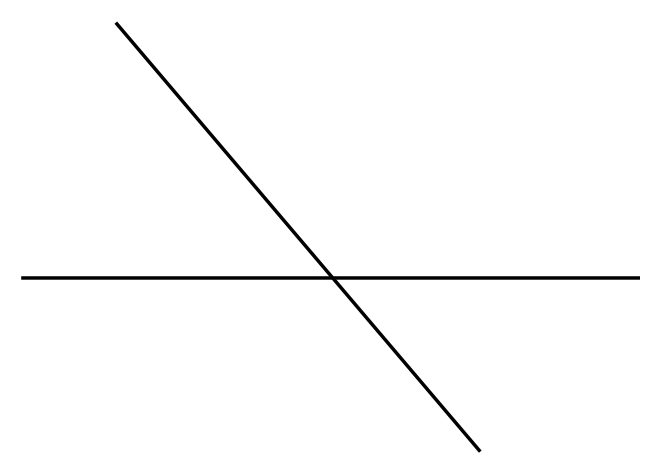

Figure 21: Schematic of the single-centreline representation of the highway network

4. Step 4 applies criteria 1 and 2, and creates a layer of points at highway section intersections of the A_U_LRS_2015 using the Feature Vertices to Points tool (see Figure 22). This point layer, Hwy_Intersection_Points, provides a map of the potential sequencing locations. 


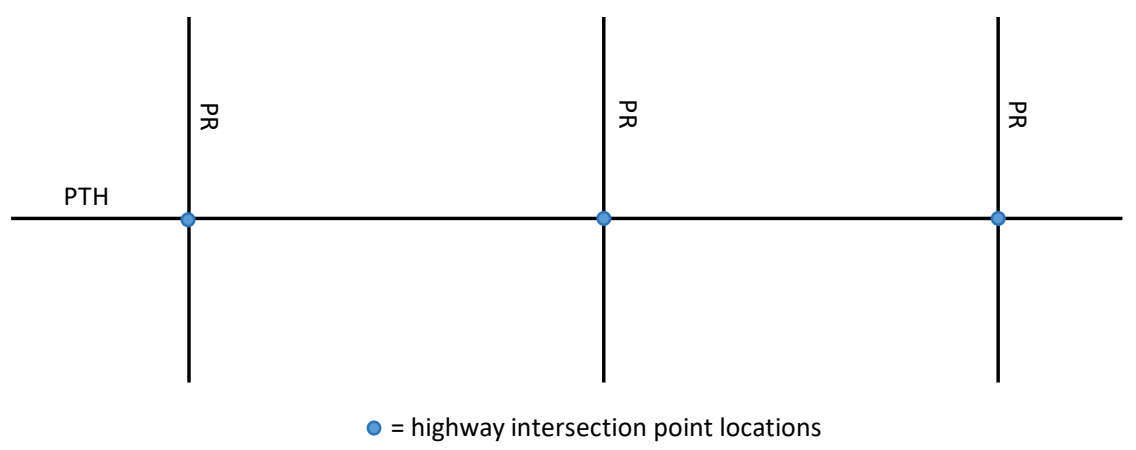

Figure 22: Illustration of creating the point layer at highway intersection locations

5. Step 5 creates a copy of the Hwy_Intersection_Points layer, naming it Export_Points. This new layer allows for changes to the intersection points.

6. Step 6 reviews the points in the Export_Points layer to ensure points were created at appropriate locations. In particular, locations where highways cross via an over/underpass but do not intersect are inspected for incorrect points. Points are removed as needed.

7. Step 7 applies criteria 3 , and adds additional points at intersections with municipal roads. Major city roads are not part of the highway network, but intersect with highways, so their intersection points must be added manually.

8. Step 8 uses the Split Line at Point tool to split A_U_LRS_2015 at the potential new sequence points and create the A_U_LRS_2015_Split layer. The input features are those in the A_U_LRS_2015 layer, and the point features are those in the Export_Points layer. Including a search radius is optional when using this tool, but is necessary in this procedure. A line is split at all points within a given search radius when one is defined; if one is not defined, the line will only be split at a single point on the line.

9. Step 9 uses the Feature Vertices to Points tool to confirm the highways were split appropriately. This tool creates a layer of segment endpoints, which is compared to 
the Export_Points layer to ensure the highways are split at the appropriate locations. If there are locations that were not split, the Split tool can be used.

10. Step 10 modifies the Manitoba_Subdivisions layer to only include cities and towns with populations over 5000, which are Brandon, Dauphin, Flin Flon, Morden, Portage la Prairie, Selkirk, Steinbach, The Pas, Thompson, Winkler, and Winnipeg. This step uses the Select by Attributes tool in the Manitoba_Subdivisions attribute table to select only the cities/towns of interest, and then exports the selection as its own layer file (Major_Manitoba_Subdivisions).

11. Step 11 applies criterion 4 and creates a layer of points at the intersection of major jurisdictional/subdivision boundaries and PTHs/PRs. The Intersect tool with Major_Manitoba_Subdivisions and A_U_LRS_2015 as the "Input Features" and "POINT" as the "Output Type" creates a layer of points (Subdiv_Intersect) at all intersections between the jurisdictional boundary polygons and the line-based highway network.

12. Step 12 inspects the locations of all Subdiv_Intersect points for appropriateness. Key considerations include:

12.1. Proximity to $P T H / P R$ intersection

12.2. Proximity to segment endpoint

12.3. Reasonableness of point location (e.g., if a jurisdictional boundary crosses a highway segment in more than one location).

Any points that are too close to PTH/PR intersections or segment endpoints, or are in an unreasonable location, are removed.

13. Step 13 uses the Split Line at Point tool to sequence the network at jurisdictional boundaries. In this step, the input features are the highway network and the point features are in the layer Subdiv_Intersect. 
14. Step 14 numerates highway sequences. The sequencing topology increases in the easterly and northerly directions; although highways may change direction, their main direction is considered for sequence numeration. Sequence numbers start at 10 and increase by increments of 10 travelling west to east or south to north along a section. Sequencing numeration starts over at 10 at the beginning of each new section. Figure 18, shown earlier, illustrates the process of sequence numeration. Numeration is performed manually on a highway-by-highway basis, starting at PTH 1 and continuing to PR 596.

14.1. To start, a field titled "Sequence" is added to the attribute table of the Sequenced_Highway_Network layer. This is performed by opening the layer's attribute table, selecting the Table Options button, and then selecting the Add Field... option. The field is given the title "SEQUENCE_NO", and should be of type short, since the sequence numbers assigned are only two digits. The field should also Allow NULL Values.

14.2. The second step of sequencing allows for editing of the highway network layer. Right click the Sequenced_Highway_Network layer, hover over Edit, and select Start Editing. This will allow highway sequences to be assigned sequence numbers individually rather than using the Field Calculator to calculate the entire field of highway sequences at once.

14.3. This step highlights the highway to be sequenced using the "Select by Attributes" query creator. The query selects features based on desirable attribute values. To start with PTH 1, the query reads "ROAD_NO = 1", and will select all features (i.e., road segments) of PTH 1.

14.4. This step assigns sequence values to the segments of PTH 1. This can be done by: 
1. starting at the first sequence and first section of the current highway, and selecting and numerating sequences while moving along the highway in the direction of increasing sections; or

2. using the attribute table to highlight the sequences that make up the first section, and numerating them in the appropriate order before proceeding to the next section.

14.5. This step repeats Steps 14.3 and 14.4 for all highways.

If any issues occurred in the segmentation process, they are identified in this process and can be remediated.

\subsection{COUNT SITE ATTRIBUTION PROCEDURE}

This section outlines the procedure developed to attribute sites and their traffic volume characteristics to highway sequences. Ideally, a count site would exist on each highway sequence, whether that be a permanent count station or a coverage count station. However, this is neither economically feasible nor efficient. In reality, three scenarios exist:

1. 1:1 Case - when exactly one count site exists on a highway sequence.

2. 0:1 Case - when zero count sites exist on a highway sequence.

3. Many:1 Case - when more than one count site exists on a highway sequence. From an attribution perspective, the ideal case is the 1:1 scenario; however not all highway sequences have exactly one count site situated on the segment. Cases where zero count sites exist on a sequence introduce gaps in data collection into the network, whereas cases with multiple count sites on a sequence introduce data redundancies, which is an inefficient use of resources. Consequently, a method to attribute count sites and traffic data to highway sequences must be developed. The principles are intended to be pragmatic and reflect current realities in Manitoba. As such, the principles apply data 
validation thresholds that have historically been used to screen traffic data within Manitoba's traffic monitoring program. For example, a threshold of plus/minus 20 percent is routinely used to screen for unusual changes at a short-duration count site from year to year.

\subsubsection{Principles for attributing count sites to sequences}

This research develops and applies three multi-part principles for attributing count sites to sequences.

\section{Principles for 1:1 Cases}

a) Count sites are attributed to the sequences on which they are situated.

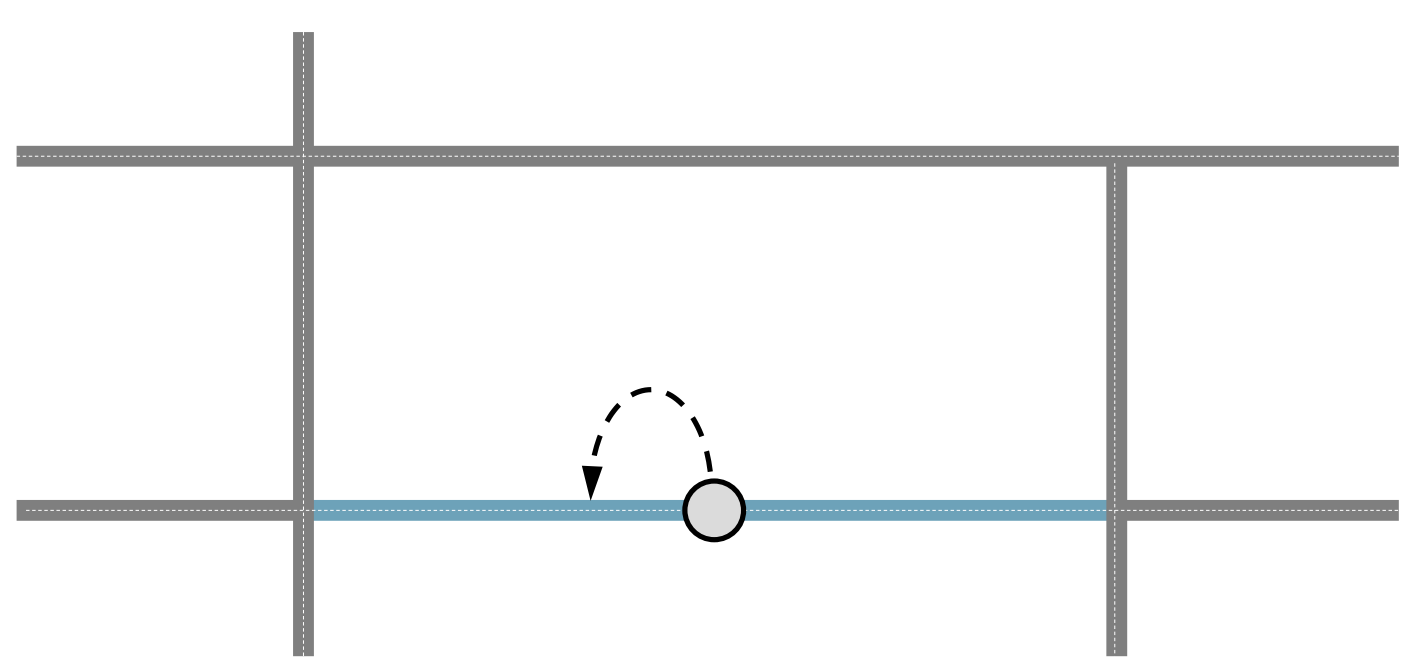

Figure 23: Example of principle 1. a)

\section{Principles for 0:1 Cases}

a) A site on an adjacent sequence may be attributed to a sequence without a site if the intersecting highway is a 600 series highway. 600 series highways are smaller/less significant than PTHs and PRs, and are typically not surveyed. For this reason, it is assumed that their 
influence on the volume of an intersecting highway is minimal. Figure 24 illustrates this principle.

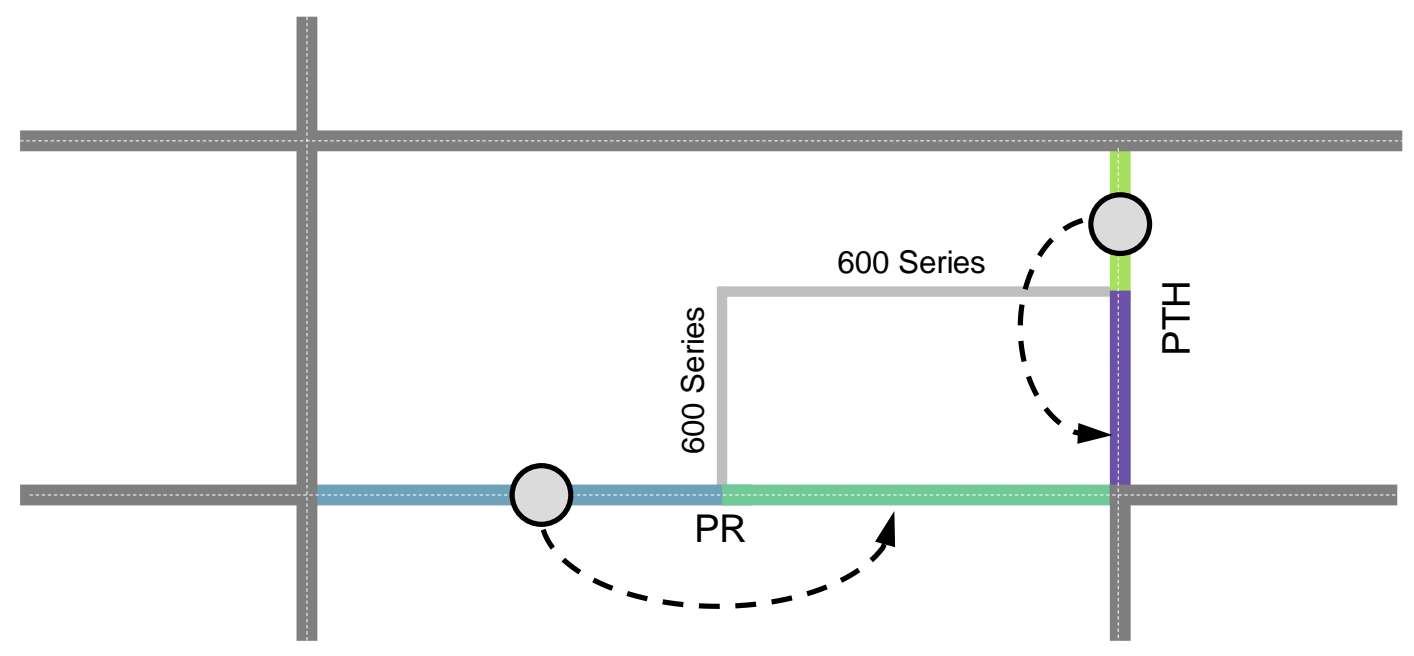

Figure 24: Example of attribution principle 2. a)

b) A count obtained on an adjacent sequence may be attributed to a sequence without a site if the intersecting highway has less than $20 \%$ of the AADT on the adjacent sequence. If the intersecting highway has more than $20 \%$ of the AADT of the adjacent sequence, attribution may still occur but the sequence is flagged for future improvements.

Figure 25 illustrates this principle.

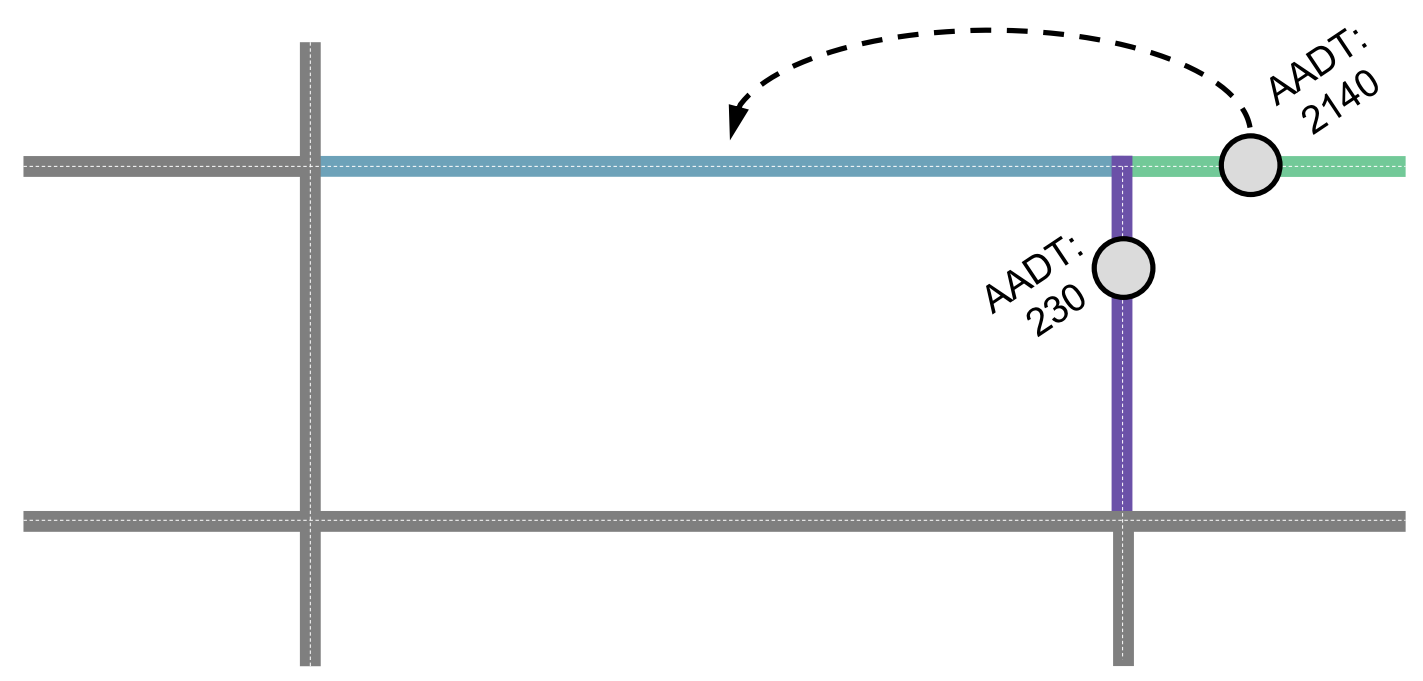

Figure 25: Example of attribution principle 2. b) 
c) A count site from an adjacent sequence may be attributed to a sequence without a site if turning movement counts show similar traffic patterns on the two sequences. Figure 26 illustrates this principle.

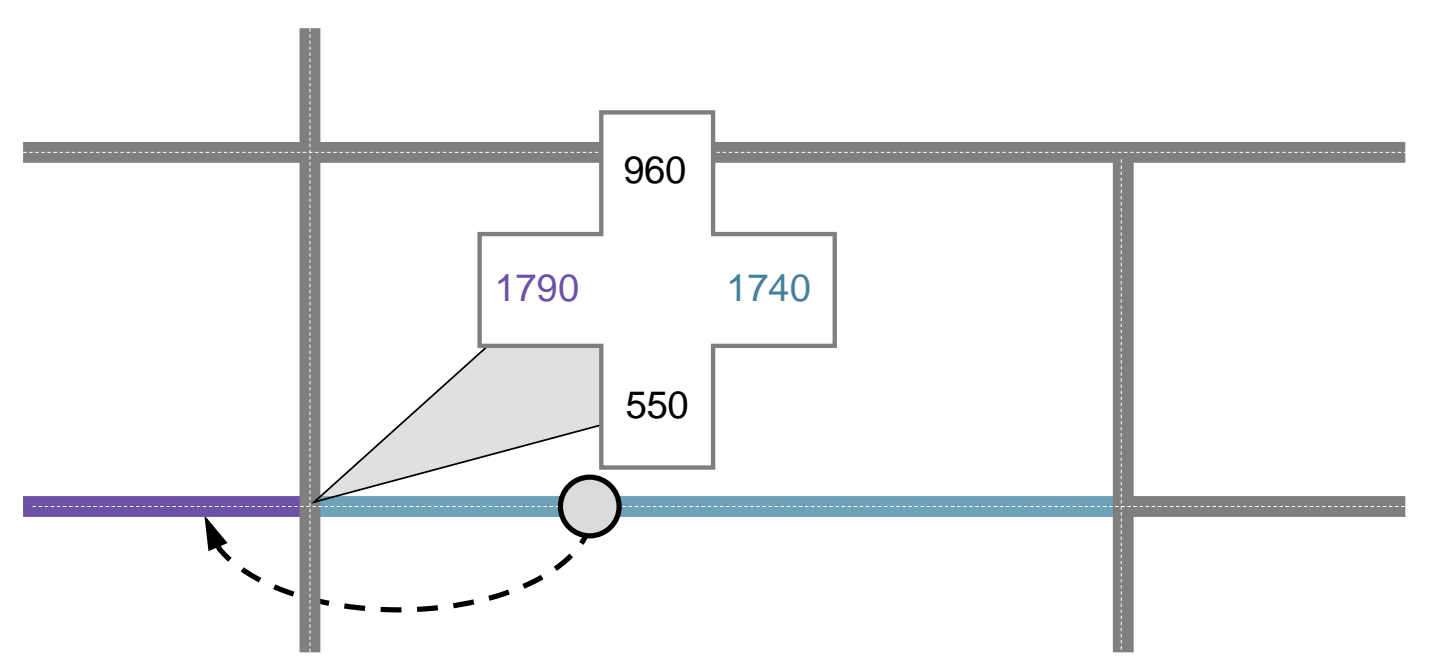

Figure 26: Example of attribution principle 2. c)

d) The count site of the adjacent sequence with the highest AADT may be attributed to the centre sequence of an offset intersection. An offset intersection is one where the highway legs in either the north/south or the east/west direction do not align. This principle is created based on flow balance, as there is a potential for traffic volumes on the centre sequence to exceed those on surrounding sequences. Figure 27 illustrates this principle. 


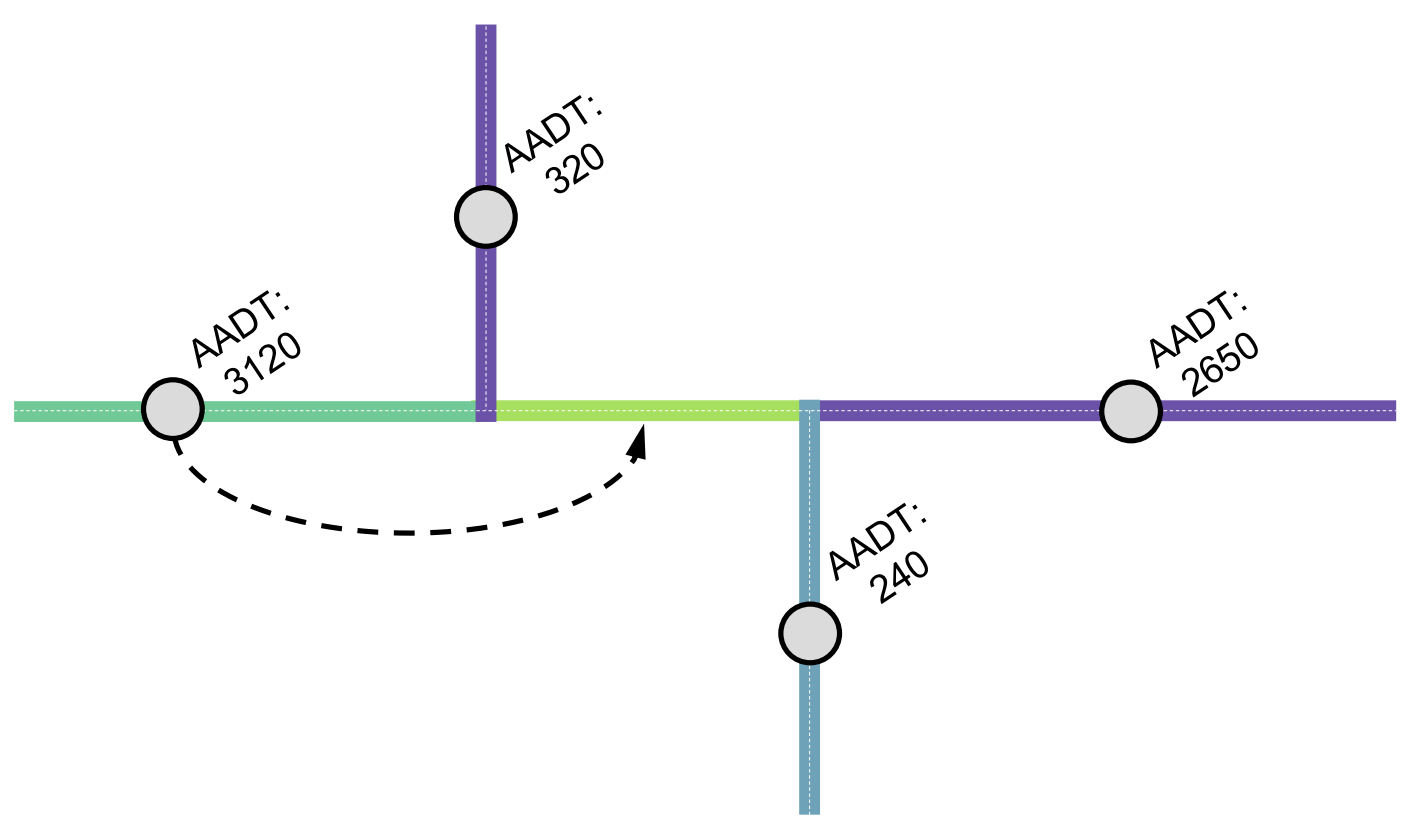

Figure 27: Example of attribution principle 2. d)

e) A sequence with a count site that has been removed may be attributed volume from the former count site with a growth factor applied. In Manitoba, traffic displays a linear growth pattern; the equation to calculate traffic volume is as follows:

$$
A A D T_{p}=A A D T_{c}+(\text { growth } \times(p-c))
$$

where:

$A A D T_{p}=\mathrm{AADT}$ of present year

$A A D T_{c}=\mathrm{AADT}$ of count year

Growth $=$ Annual growth in vehicles per year

$p=$ Present year

$c=$ Count year

Figure 32 illustrates this principle. 


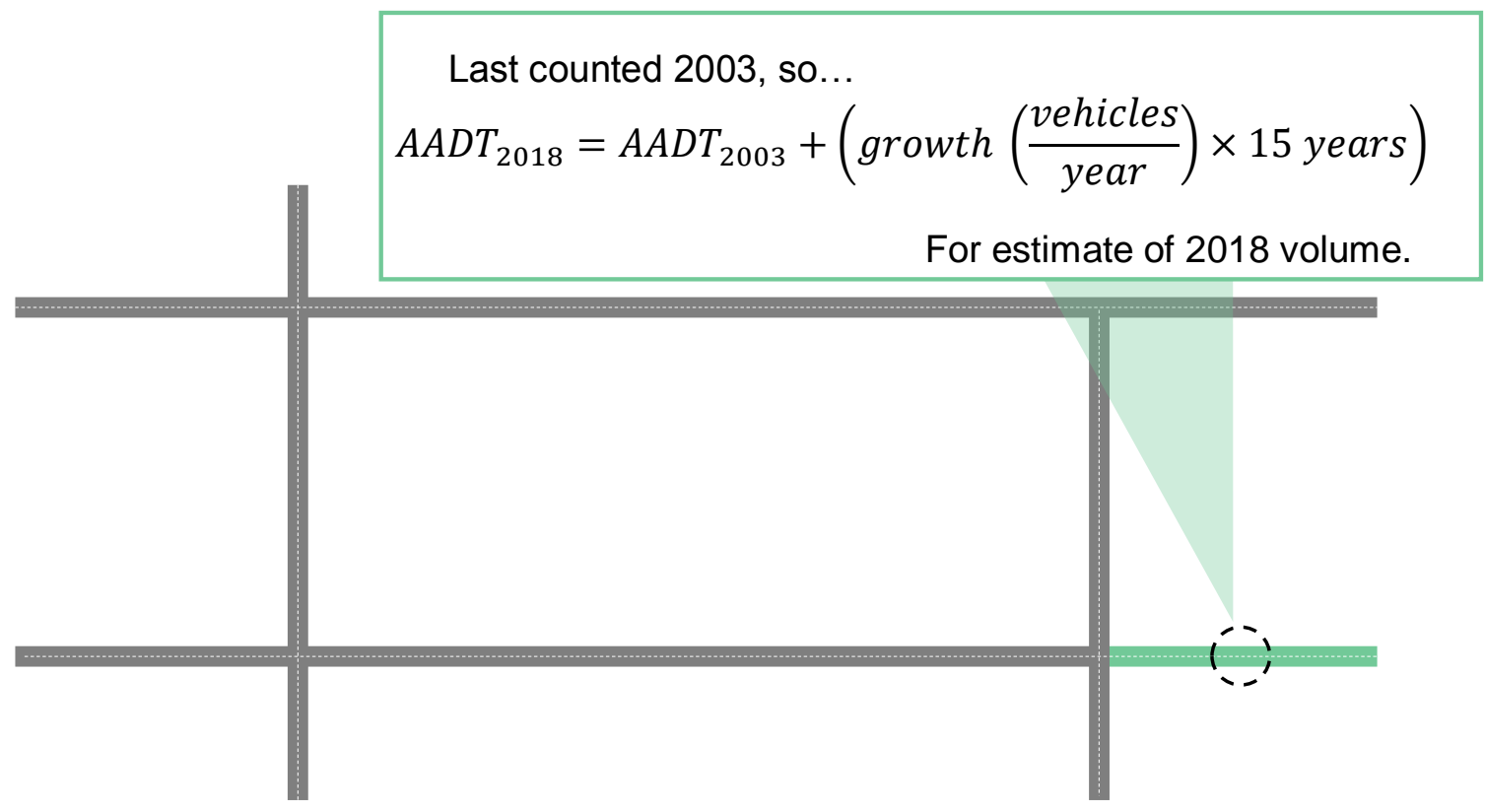

Figure 28: Example of principle 2. e)

f) A count site from an adjacent sequence may be attributed to a sequence if the two sequences belong to different traffic control sections. Sections are subdivided at locations such as divided/undivided transitions, or certain built infrastructure including bridges. Such locations do not necessarily introduce traffic volume fluctuations and so count sites may be attributed over section endpoints. Figure 29 gives an example of this principle. 


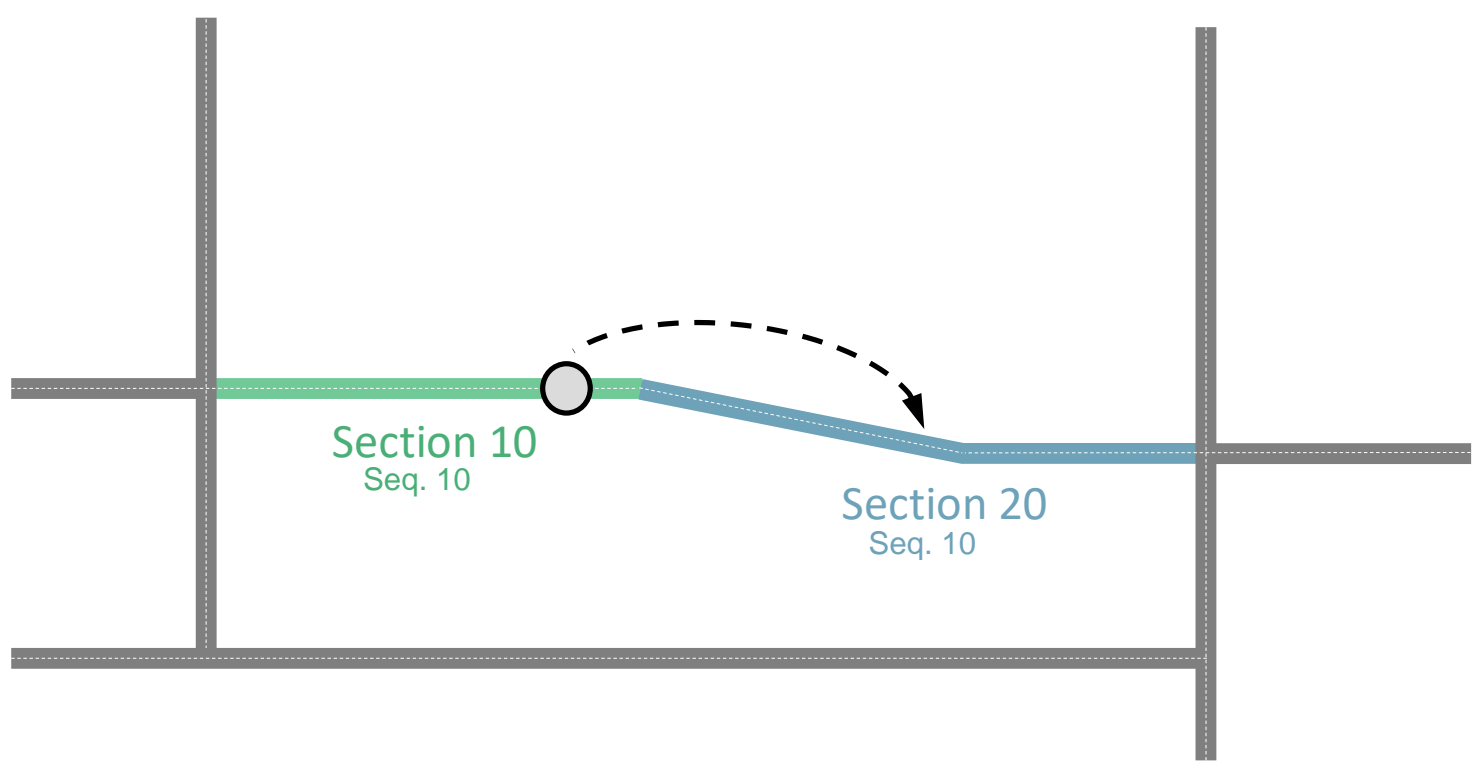

Figure 29: Example of attribution principle 2. $\mathrm{f}$ )

\section{Principles for Many:1 Cases}

a) Continuous count sites take precedence over short duration count sites. Continuous count sites collect continuous traffic data for an entire year (unless equipment malfunctions); however, short-duration count sites only collect data for 48 hours of the year. Consequently, continuous count site data are more reliable and preferred for attribution. Figure 30 illustrates this principle.

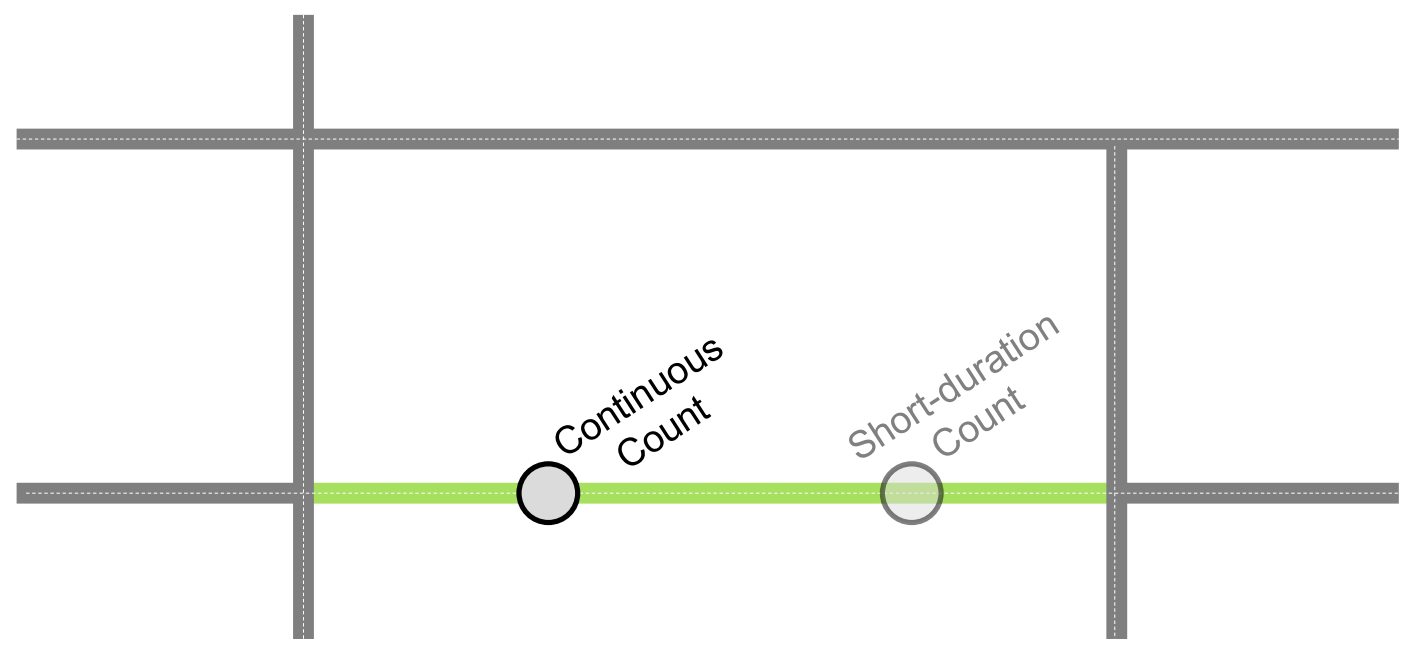

Figure 30: Example of attribution principle 3. a) 
b) The site with the most recent data takes precedence over others. If traffic patterns are similar between the count sites, the site with the most recent data are attributed to the sequence.

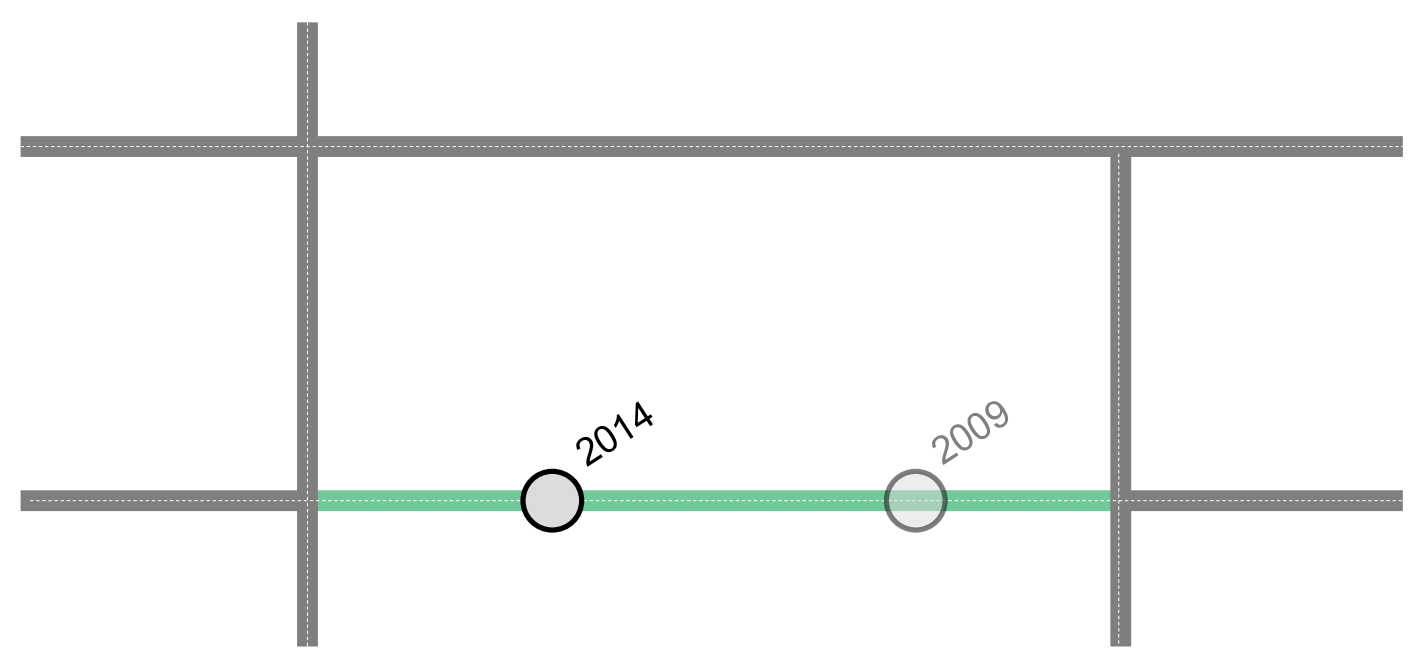

c) When AADTs $\leq 500$ : if the count site with greatest volume has an AADT less than twice that of the lowest volume site, the highest volume count site is attributed to the sequence and the remaining count sites are flagged. Figure 31 illustrates this principle.

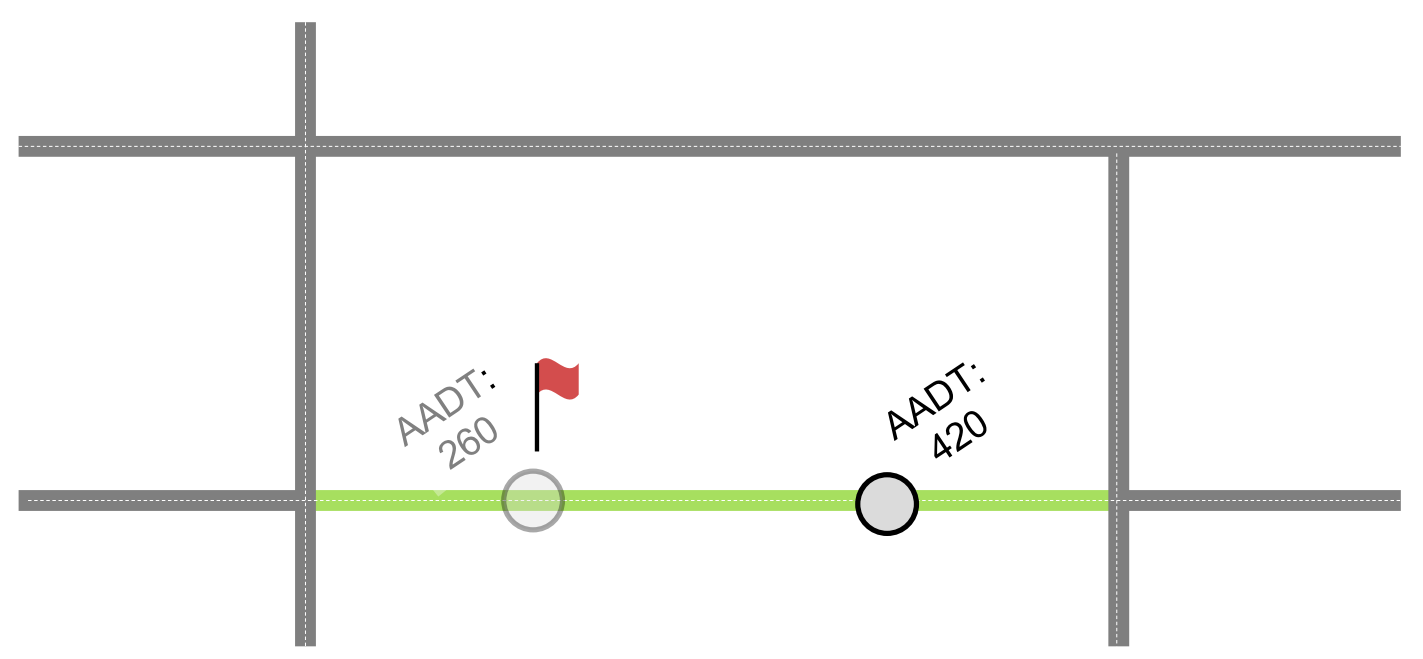

Figure 31: Example of attribution principle 3. c) 
d) When AADTs > 500: if the count sites have AADTs that are less than $20 \%$ different from one another, the highest volume count site is attributed to the sequences and the remaining count sites are flagged. Figure 32 illustrates this principle.

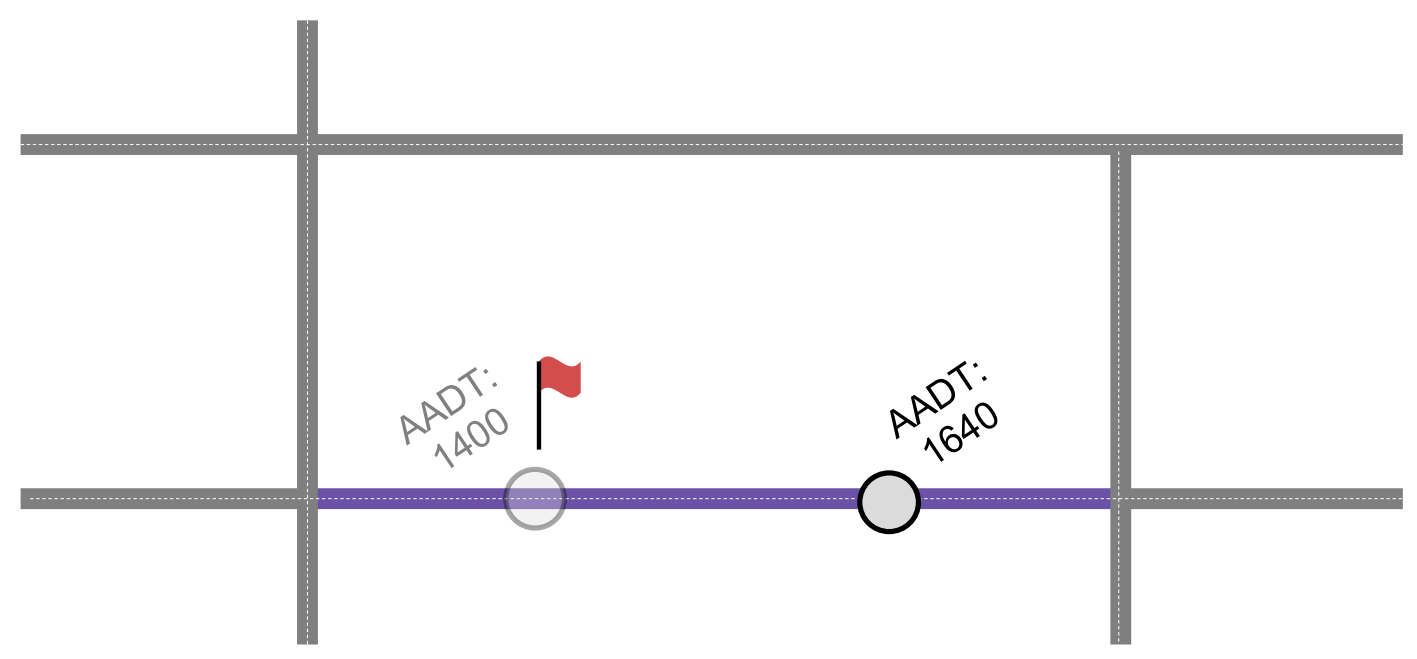

Figure 32: Example of attribution principle 3. d)

Note that for all cases, sequences within city boundaries are flagged due to the complexities of urban traffic monitoring. However, city sequences are still attributed count sites based on the aforementioned principles. Figure 33 gives an example of this.

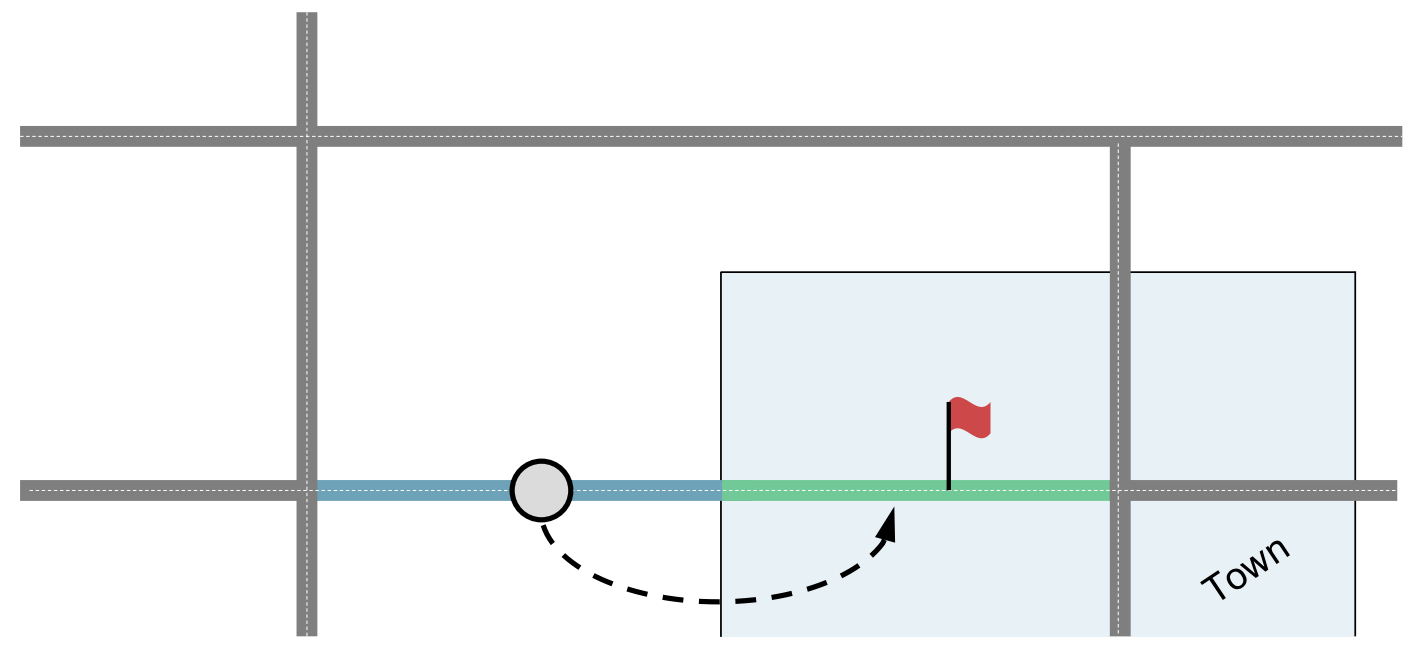

Figure 33: Example of attribution principle 4. b) 
Additionally, sequences that do not follow any of the previous principles are flagged for future improvements to the traffic monitoring program. These sequences are attributed the same count sites as in previous years. Figure 34 gives an example of this.

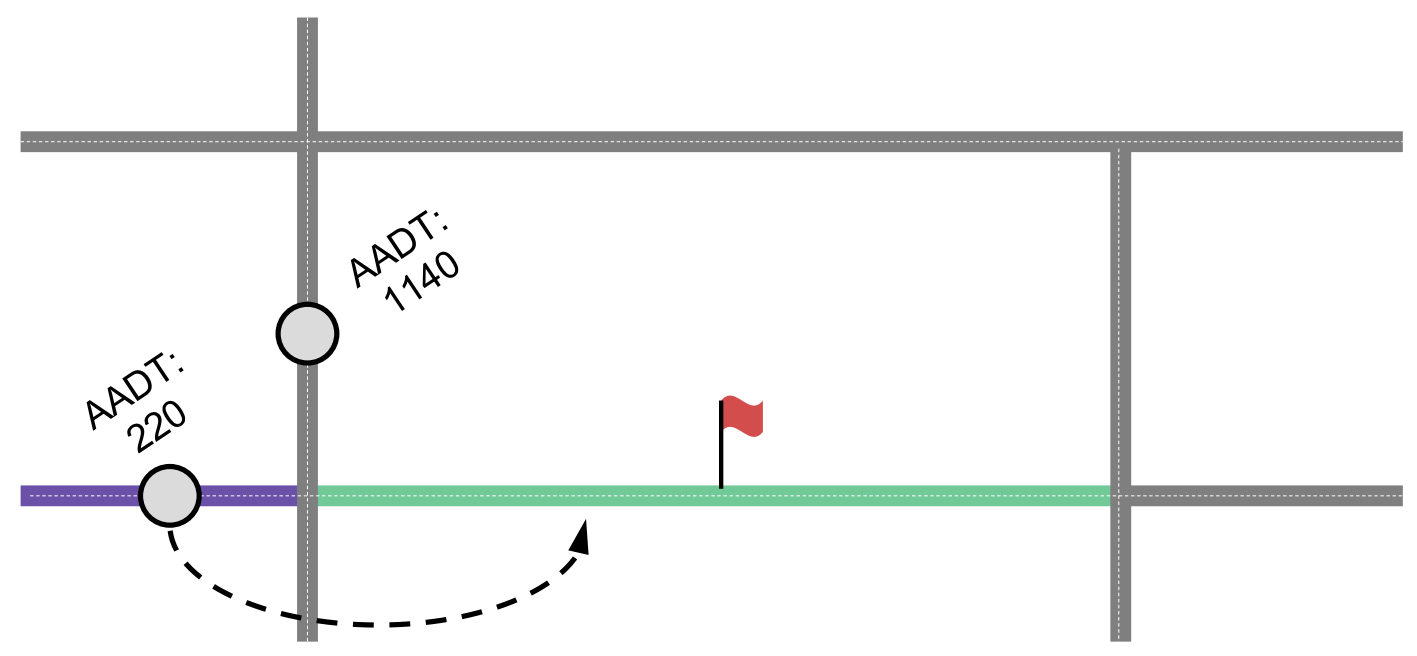

Figure 34: Example of attribution principle 4. c)

Also note that there are implications in choosing to attribute the highest AADT site in principles $2 \mathrm{e}$ ) and $3 \mathrm{c}$ ) and d). Choosing the highest AADT is essentially the conservative choice, and allows for future changes to not be under designed.

\subsubsection{GIS Functions}

Similar to the sequencing methodology, ArcGIS is used to attribute traffic volume to the highway network. Figure 35 shows this procedure. 


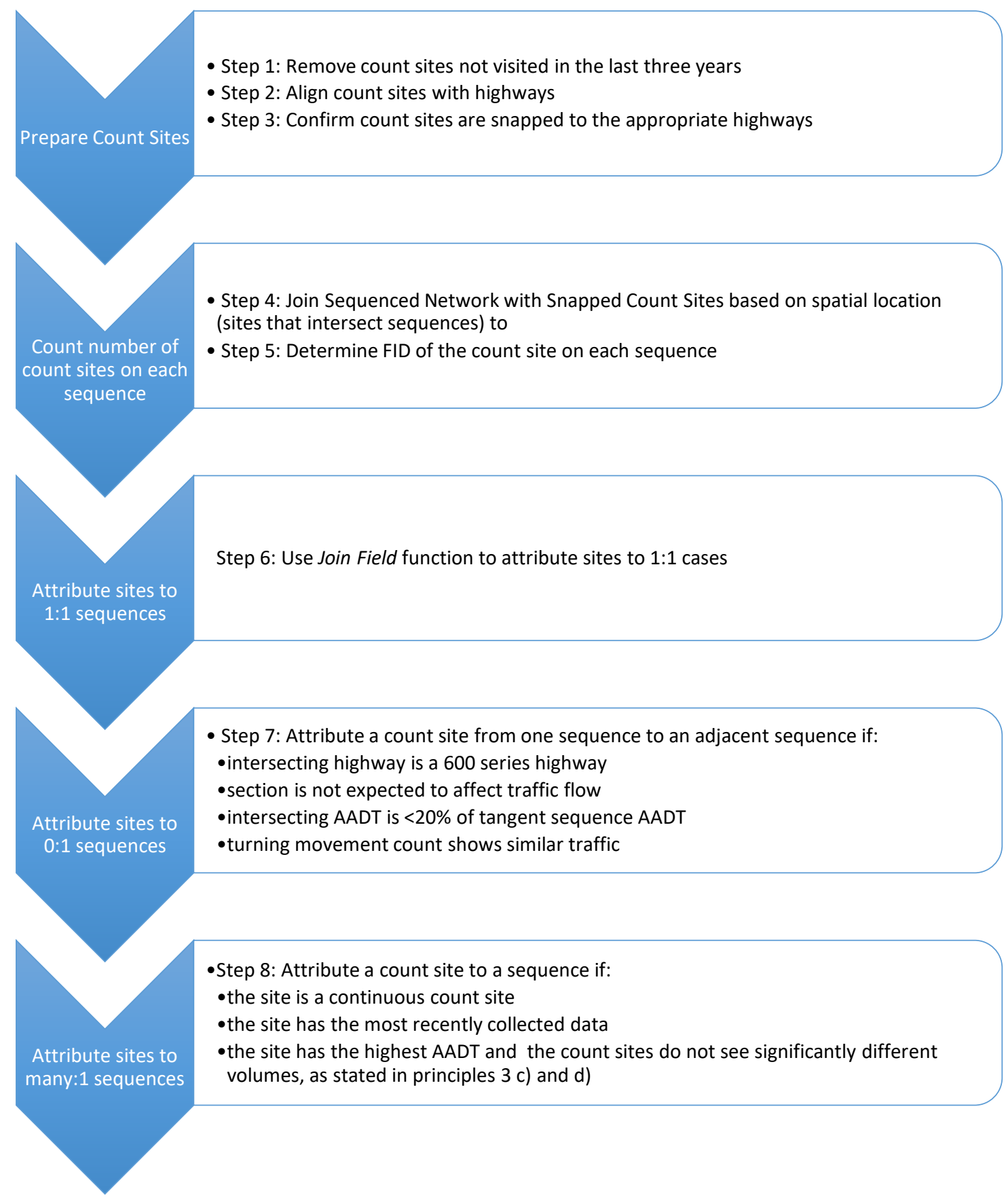

Figure 35: Flowchart of count site attribution procedure

A more detailed description of the attribution procedure follows:

1. Step 1 removes count sites that have not been counted in the past three years. To do so, a table of all coverage count sites is added to the ArcMap file and joined with the Count_Sites layer by Station Number. The sites with Null values in the additional 
columns means the site has not been counted in the past three years and can be deleted. Deleting the sites pre-2014 by hand confirms that the correct sites are being deleted. Sites that have been removed in the past three years are also deleted from the Count_Sites layer in this step.

2. Step 2 aligns count sites with highways. The highway network file and the count sites file are created separately, resulting in count sites, represented as points, rarely aligning with the highway network. This step snaps count site points to their nearest highway sequence.

a) Use the Near tool to find the points on the highway network located nearest to the counts sites. In this step, the Input Features are those in the Count_Sites layer, and the Near Features are those in the Sequenced_Network layer. The Location checkbox must be selected to give the $x$ - and $y$ - coordinates of the nearest location. This gives four new fields to the Count Sites layer: NEAR_FID, which gives the FID of the nearest sequence; NEAR_DIST, which gives the distance to the nearest sequence; and NEAR_X and NEAR_Y, which give the $\mathrm{X}$ and $y$-coordinates of the nearest point on the nearest sequence.

b) Export the attribute table of the Count Sites layer, and save it as a dBASE file. When prompted, select "Yes" to add the table to the current map.

c) Right click on the new table added to the map and select Display XY Data. In the Display $X Y$ Data window, set $X$ Field to NEAR_X and Y Field to NEAR_Y. This will create a new event layer with points at the locations of NEAR_X and NEAR_Y coordinate pairs. Export this event as its own layer to save it. This layer is saved as Snapped_Count_Sites.

3. Step 3 visually compares the Count_Sites layer and the Snapped_Count_Sites layer for major differences. Count sites may have been incorrectly snapped to a highway in Step 2 when in actuality they are located on an access road or a highway that is 
no longer under provincial jurisdiction. The sites that do not exist on the LRS are manually moved back to their appropriate locations. Figure 36 shows an example of a site snapping to a location on the LRS though it is realistically on a separate $\mathrm{road} / \mathrm{highway}$ not in the LRS. The green dot on PR 236 of the 2005 highway network is the initial position of the count site, and the pale green dot located on PTH 6 of the present highway network is where it is incorrectly snapped to in Step 2.

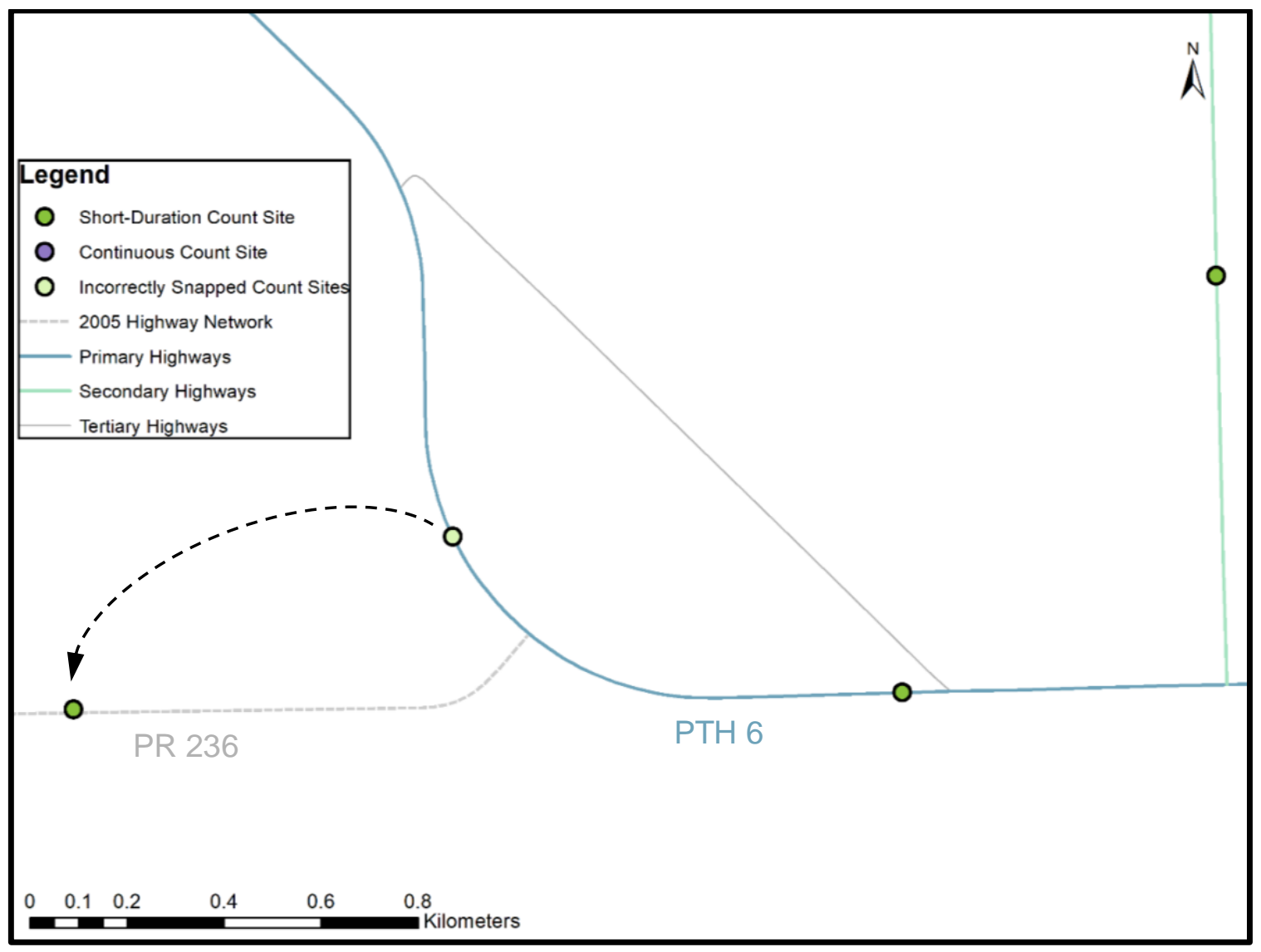

Figure 36: Error in snapping counts to nearest highway on the highway network

4. Step 4 uses the Join function to count the number of count sites that intersect the highway network sequences. The join is performed on the Sequenced_Network layer, which is joined with data from another layer based on spatial location, namely the Count_Sites_Snapped layer.In the window, select the option to Join data from another layer based on spatial location and select the Count_Sites_Snapped layer. 
Under the Each line will be given a summary of the numeric attributes of the points that are: option, select intersected by it. This join creates a new layer, called Sites_Sequences_Join. In the attribute table of this new layer, there will be a Count field, which stores the number of Count Sites intersected by each sequence, whether that is zero (or "Null"), one, or more than one.

5. Step 5 uses the Near function to determine the FID of the count site on each sequence. If there is no site on a sequence, the FID value will be -1 . If there is more than one site on a sequence, a site is randomly chosen to be the near site. For this reason, use the field calculator to assign a NEAR_FID value of zero to sequences with zero or more than one count site, which is known from the previous step.

6. Step 6 applies Principle 1 and uses the Join Field function to attribute sites to the 1:1 cases. The join is performed using the NEAR_FID field in the Sites_Sequences_Join layer, and the FID field from the Count_Sites_Snapped layer. This step results in having station numbers attributed to sequences under the NEAR_STN field rather than the station FID values.

7. Step 7 applies Principle 2 to attribute count sites to sequences with zero count sites. In this situation, transfer a count site from one sequence to an adjacent sequence if:

a) the sequence was split at a 600 series highway;

b) the two sequences are from two different sections but the sectioning location has little to no impact on traffic volumes;

c) the intersecting highway has less than $20 \%$ AADT relative to the selected sequence; or,

d) the turning movement count shows similar volumes occurring on the sequence and its adjacent sequence.

A count site is attributed under the NEAR_STN field of a sequence. Locations where none of these rules apply are flagged as improved counting strategies are 
necessary. Note that sequences that follow principle 2 e) are not attributed flow until the evaluation and last steps procedure, at which point all other sequences are also attributed flow based on their attributed count sites.

8. Step 8 applies Principle 3 to attribute count sites to sequences with two or more count sites. In this many sites to one sequence scenario, attribute the site that:

a) is a continuous count site;

b) has the highest AADT when: the greater AADT site is less than double the volume of the lesser site(s) and AADTs are less than or equal to 500 vehicles/day, or less than $20 \%$ difference and AADTs are greater than 500 vehicles/day; or,

c) has the most recent data.

A count site is attributed under the NEAR_STN field of a sequence. If none of the above scenarios apply, flag the sequence for further investigation.

\subsection{EVALUATION PROCEDURE AND FINAL STEPS}

Upon completion of the sequencing and attribution procedures, it is apparent that some sequences do not meet any of the attribution principles defined in Section 3.3. This section outlines a procedure to remediate these issues.

\subsubsection{Evaluation Procedure}

The most common issue involves a sequence that has more than one count site with significantly different traffic volume estimates. Consequently, these sequences require further subdivision. Sequences are evaluated on a case-by-case basis to determine potential locations of traffic volume change and further subdivide them into shorter sequences. This process applies engineering judgement to determine where such locations may be, and include the following: 
1. Highway vertices - sometimes highways will take a 90 degree turn, and another road that is not under provincial jurisdiction continues straight, which may cause traffic fluctuations. For this reason, 90-degree turns may be a point of subdivision.

2. Major origins/destinations - in some instances, a major destination exists along a highway, which could cause traffic fluctuations at that particular location. For this reason, this may be a point of subdivision.

3. Change in road surface type - paved roads are often more desirable to drive than gravel roads, and therefore may be a significant cause of traffic volume changes. For this reason, a change in road surface type may be a point of subdivision.

4. Road leading to a town/destination - when a road intersecting with a highway leads to a town, it is expected to cause traffic volume fluctuations along the highway. For this reason, an intersecting road may be a point of subdivision.

5. Towns with populations $<5000-$ a town is likely to influence traffic volume along a highway, therefore towns (in addition to those considered in Section 3.2) may be a point of subdivision.

If it is unclear where the change in traffic volume may occur, the segment is split in half so each sequence has equal length. Count sites are then attributed to sequences based on the principles set out in Section 3.3.

Once each sequence has an attributed count site (or follows principle 2 e) of attribution), traffic volumes from count sites in the form of AADT can be attributed to sequences.

\subsubsection{GIS Functions}

Similar to the sequencing attribution procedures, ArcGIS is used to attribute traffic volume to the highway network. Figure 37 shows this procedure. 
Step 1: Review sequences with multiple count sites for potential locations of traffic flow change and measure location

Review and subdivide

sequences with

multiple count sites

Step 2: Subdivide sequences at locations of traffic flow change

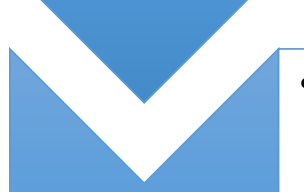

- Step 3: Update sequence attributes:

- number of count sites on sequence

-nearest count site to sequence

Update attributes of

newly subdivided

sequences

-sequence number

- sequence number of surrounding sequences

- Step 4: Calculate growth for sequences with recently removed count sites

- Step 5: Attribute flow to sequences based on nearest count site or growth rate

- Step 6: Sort table by CS_KEY and SEQUENCE_NO

Complete Sequenced

Network Layer

- Step 7: Calculate start and end km of highway sequences

Figure 37: Flowchart of evaluation procedure

A more detailed description of the evaluation procedure follows:

1. Step 1 examines a segment with multiple count sites for potential locations of traffic volume change. Google Maps and Google Street View are used to determine where such locations may be. Once a location is determined, the Measure tool in Google Maps is used to measure the distance from the start or end point of the segment to the desired location.

2. Step 2 uses the Split function in the editor toolbar to subdivide the segment. To split the segment at some distance from the start/end point, click the Editor dropdown button and select Split. Enter the distance measured and select whether that distance is from the start or end point of the segment. To select the split point on the segment, click the Split button on the toolbar and click on the segment in the location of the desired split. 
3. Step 3 updates the attributes of the new sequences, including the number of count sites on the sequence, the count site attributed to the sequence, and the sequence number. Step 3 also re-numerates surrounding sequences to account for the new sequences that were added when segments were split.

4. Step 4 creates growth factors for sequences that are attributed flow from count sites that have been removed as in principle 2 e). It calculates the average growth in vehicles per year using the available data. For continuous count sites, average growth is calculated by summing the difference in AADT between years and dividing the summed difference by the number of years. Traffic volume growth for continuous count sites is calculated using the following formula.

$$
\text { Growth }=\frac{\sum_{i=1}^{n-1}\left(A A D T_{i+1}-A A D T_{i}\right)}{n}
$$

Where:

Growth $=$ traffic volume growth in vehicles/year

$\mathrm{n}=$ number of years with available traffic volume data

AADT = annual average daily traffic of a given year

For coverage count sites, average growth considers that sites are not surveyed every year, so AADT differences between counts are divided by the number of years between count years before dividing by the number of count years. Traffic volume growth for short-duration count sites is calculated using the following formula.

$$
\text { Growth }=\frac{\frac{\sum_{i=1}^{n-1}\left(A A D T_{i+1}-A A D T_{i}\right)}{\text { count frequency }}}{n}
$$

Where:

Growth $=$ traffic volume growth in vehicles/year

$\mathrm{n}=$ number of years with available traffic volume data

AADT = annual average daily traffic of a given year 
Count frequency $=$ the number of years between coverage counts

5. Step 4 attributes volume to sequences based on the nearest site attributed to each sequence. It uses the Copy Rows function, which gives Object IDs (OIDs) to all rows of the Station_Volumes table, which contains all count sites by number and their most recent AADT estimates. It then uses the Join Field function to join data from the Station_Volumes table.

6. Step 6 uses the Sort tool to reorder the attribute table of the Sequenced_Highway_Network since after highway segmentation occurs, the highway sequences are not appropriately ordered (west to east or north to south) in the attribute table. In this step, the Input Dataset is the Sequenced_Highway_Network, and the fields to sort by, in ascending order, are CS_KEY and SEQUENCE_NO. The Output is named Ordered_Highway_Network. It is desirable to sort the table by highway control section key and sequence to allow for correct start and end kilometre calculations.

7. Step 7 calculates the start $\mathrm{km}$ and end $\mathrm{km}$ of each highway sequence. Two fields are added to the attribute table to store the start and end kilometres of each sequence. The start kilometre field can be calculated using Python code; Appendix C provides the code. End kilometre is calculated by adding the sequence length to the start kilometre.

\subsection{SUMMARY}

This chapter outlined procedures for subdividing the highway network into sequences and for attributing count sites to highway sequences. It also outlined the evaluation of the sequenced and attributed highway network, introducing additional segmentation points, and assigning traffic volume to the highway network. Finally, the chapter 
described finalizing the GIS file, including calculating sequence start and end kilometre measurements. 


\section{ANALYSIS AND DISCUSSION}

This chapter discusses the results of the sequencing and attribution procedures as well as the evaluation and additional sequencing. This chapter also discusses key findings related to future data collection improvements.

\subsection{SEQUENCING}

The initial highway network file consisted of 1414 highway sections (including 600 series highways) as Figure 16 in Section 3.1.1 shows, or 1075 highway sections excluding 600 series highways.

Table 4 gives the results of the sequencing procedure, showing the number of sequences after each criterion. Figure 38 shows the sequenced highway network.

Table 4: Summary of sequencing procedure

\begin{tabular}{|c|c|c|}
\hline Criterion & $\begin{array}{c}\text { Number of Sequences } \\
\text { (including 600 Series) }\end{array}$ & $\begin{array}{c}\text { Number of Sequences } \\
\text { (excluding 600 Series) }\end{array}$ \\
\hline Initial & 1414 & 1075 \\
\hline $1 \& 2$ & 2313 & 1918 \\
\hline 3 & 2315 & 1920 \\
\hline 4 (Total) & 2365 & 1970 \\
\hline
\end{tabular}

Notes:

- Criterion 1 segments the highway network at PTH/PR intersections.

- Criterion 2 segments the highway network at intersections with 600 series highways.

- Criterion 3 segments the highway network at major municipal roads.

- Criterion 4 segments the highway network at intersections with urban boundaries. 


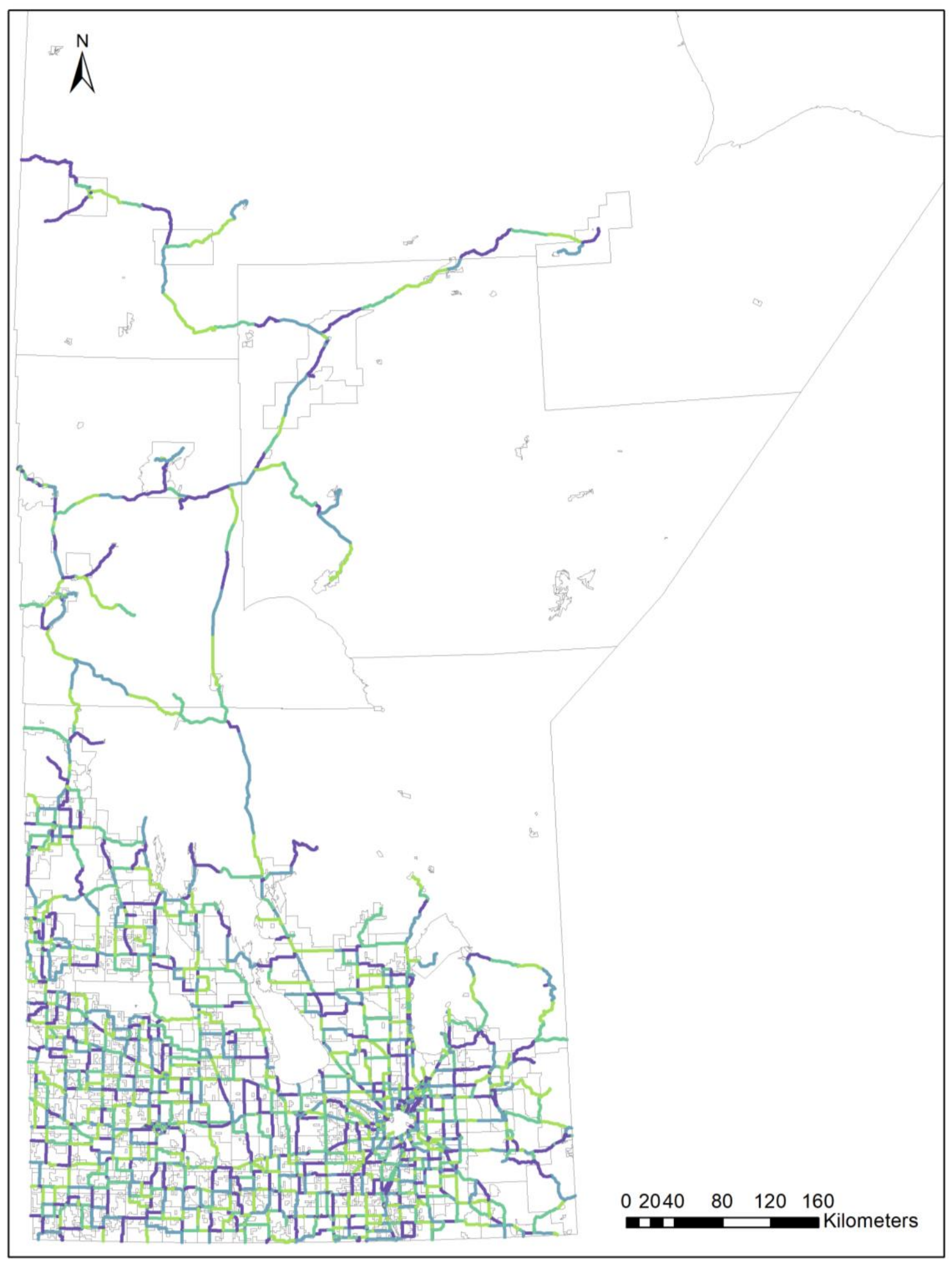

Figure 38: Sequenced highway network after first stage of sequencing 
A more detailed discussion of the sequencing procedure results follows:

- Criteria 1 and 2 of the sequencing methodology split the highway network at highway intersections. These criteria introduced 1390 intersection locations to split highway sections into sequences. This excludes locations where highways pass over or under one another, as they are not intersection locations and traffic volume does not change. Of these, 489 points are located at intersections between PTH/PTH and PTH/PR, which are already locations of section breaks, and 58 points are at 600 Series section endpoints. Considering this, Criteria 1 and 2 introduced 843 new sequencing locations at PTH/PR, PTH/600 Series, PR/PR and PR/600 Series intersections to produce 1918 sequences (excluding 600 Series highways). Figure 39 represents the 1390 locations of highway intersections as the purple-coloured points. 


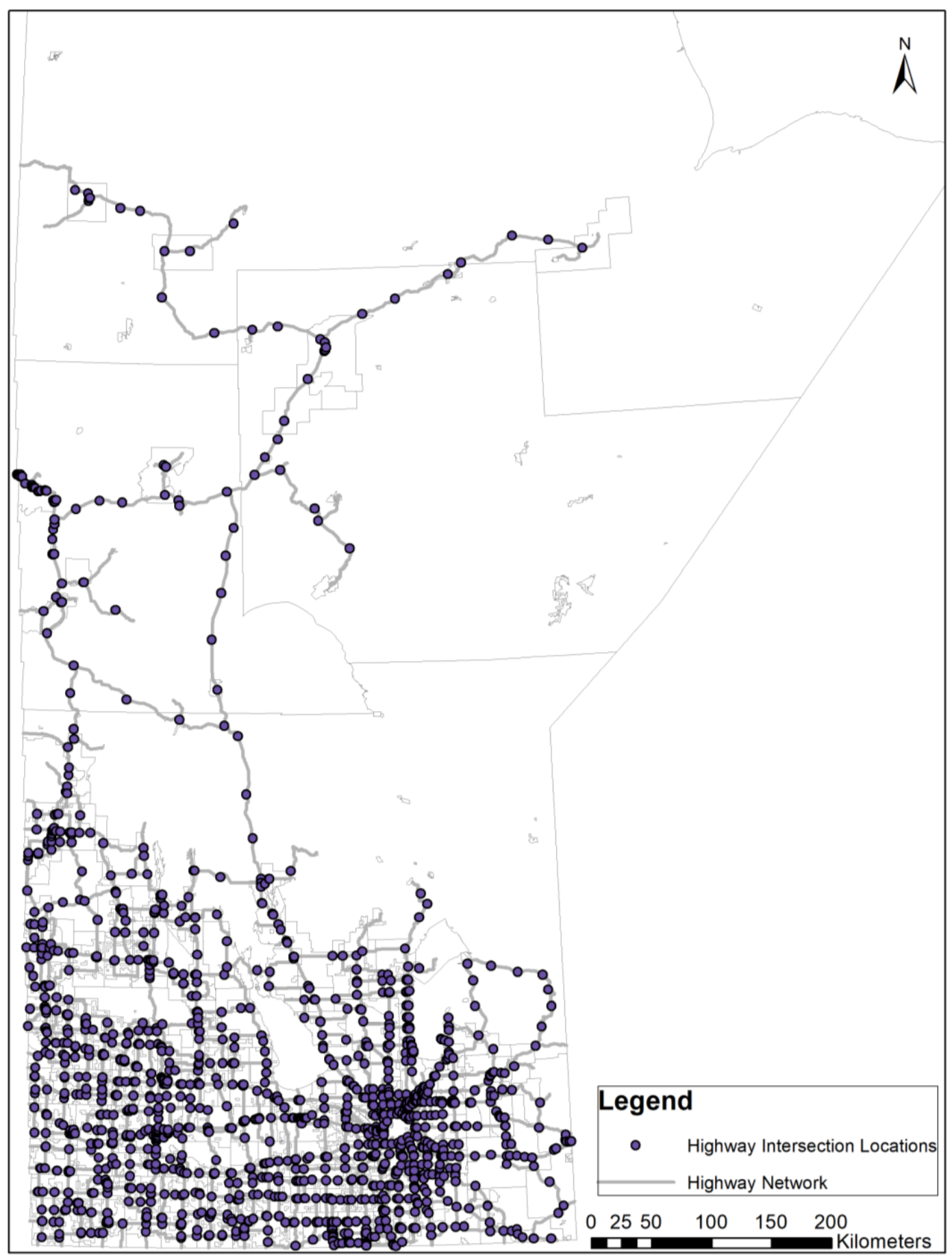

Figure 39: Highway intersections represented as points (Criterion 1 \& 2)

- Criterion 3 of the sequencing methodology split the highway network at intersections with major municipal roads. This criterion only introduced two 
additional sequencing locations at an intersection between a PTH and major Winnipeg roads. This is because most major roads in cities throughout the province are also either PTHs or PRs. These points are located at the intersection of PTH 100, also known as the Perimeter Highway, and Kenaston Boulevard, and the intersection of PTH 100 and St. Anne's Road. This principle added two more sequences for a total of 1920 sequences (excluding 600 Series highways). Figure 40 displays the split location as a purple-coloured point.

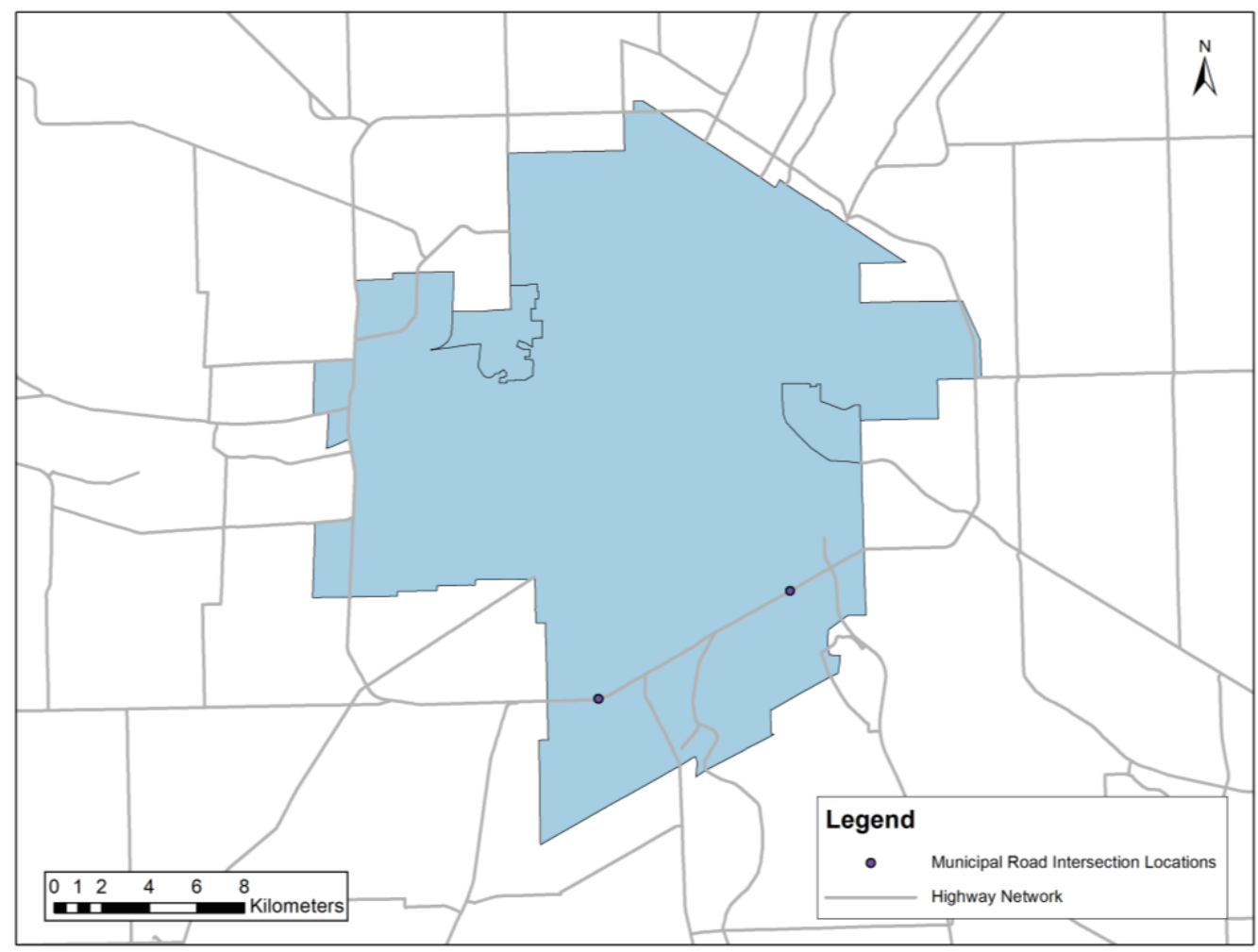

Figure 40: Municipal road/highway intersection locations (Criterion 3)

- Criterion 4 of the sequencing methodology split the highway network at intersections between highways and urban boundaries. This criterion introduced 112 potential locations for sequencing at intersections between major municipal boundaries and the highway network. Upon inspection of the boundary intersection locations, 62 were removed and the remaining 50 were used for 
sequencing. The 62 locations that were removed were a result of close proximity to other sequencing locations, and including them would produce inconveniently short highway sequences. The addition of 50 sequencing locations yielded a total of 1970 sequences (excluding 600 Series Highways). Table 5 gives the number of highway/municipal boundary intersection points by city and the number used in sequencing. Figure 41 displays highway/municipal boundary intersection points by city, with the green coloured points representing those that were unused and the purple coloured points representing those that were used.

Table 5: Number of highway/municipal boundary intersection points by city

\begin{tabular}{|l|l|l|}
\hline \multicolumn{1}{|c|}{ City } & $\begin{array}{c}\text { \# Highway/Boundary } \\
\text { Intersection Points }\end{array}$ & $\begin{array}{c}\text { \# Highway/Boundary } \\
\text { Intersection Points Used }\end{array}$ \\
\hline Brandon & 19 & 9 \\
\hline Dauphin & 8 & 4 \\
\hline Flin Flon & 10 & 4 \\
\hline Morden & 4 & 4 \\
\hline Portage la Prairie & 10 & 4 \\
\hline Selkirk & 6 & 4 \\
\hline Steinbach & 4 & 2 \\
\hline The Pas & 14 & 8 \\
\hline Thompson & 5 & 1 \\
\hline Winkler & 4 & 4 \\
\hline Winnipeg & 28 & 6 \\
\hline Total & $\mathbf{1 1 2}$ & $\mathbf{5 0}$ \\
\hline
\end{tabular}




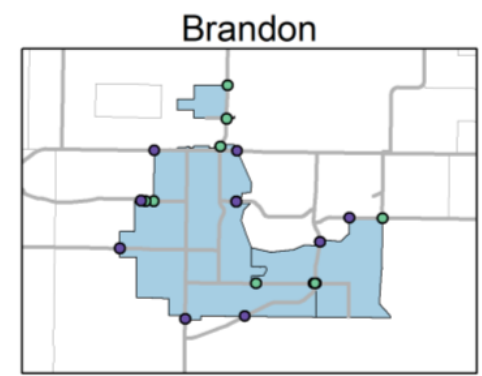

Portage la Prairie

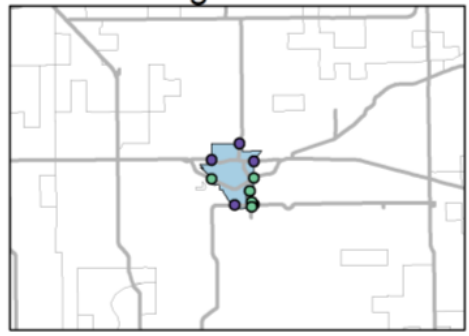

The Pas

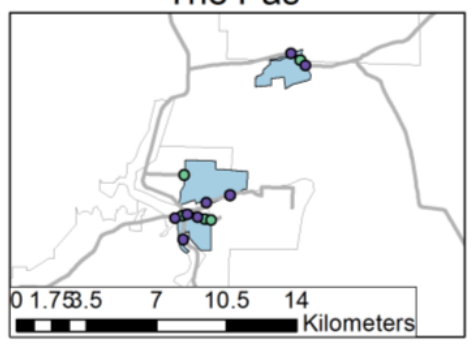

Dauphin

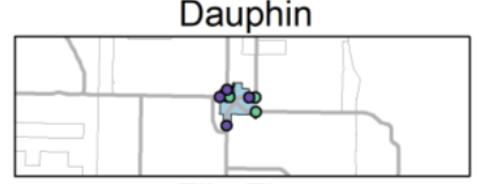

Flin Flon

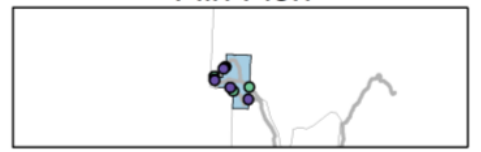

Selkirk

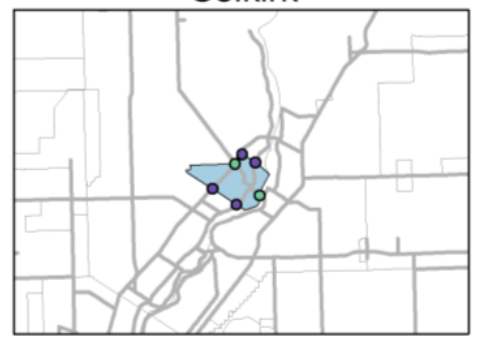

Thompson

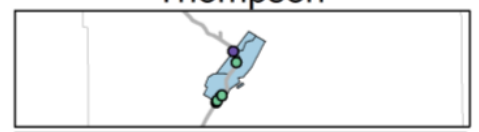

Legend

- Used Boundary/Highway Intersection Locations

Unused Boundary/Highway Intersection

- Locations
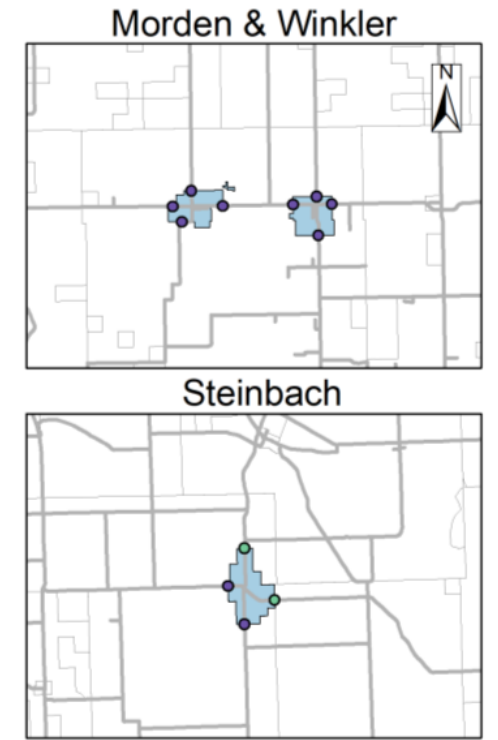

Winnipeg

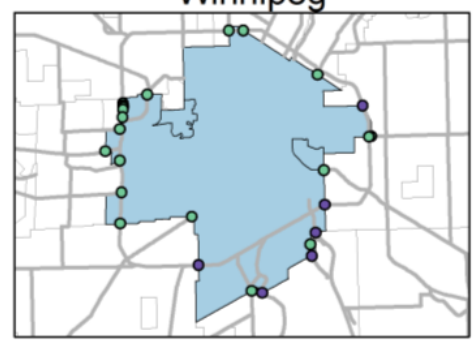

Figure 41: Boundary/highway intersection locations by city

\subsection{ATTRIBUTION}

Upon completion of the first round of sequencing, $629(31.9 \%)$ of the 1970 total sequences (excluding 600 series) initially contained 0 count sites, 974 (49.4\%) initially contained 1 count site, and 367 (18.7\%) initially contained more than 1 count site. Table 6 summarizes the network based on the number of count sites per sequence.

Table 6: Summary of highway network by case

\begin{tabular}{|l|l|l|}
\hline Case & Total Length $\mathbf{( k m )}$ & \% of Network \\
\hline $\mathbf{0 : 1}$ & 2472.891 & 13.90 \\
\hline $\mathbf{1 : 1}$ & 8627.238 & 48.51 \\
\hline Many:1 & 6685.851 & 37.59 \\
\hline Total & 17785.98 & 100.00 \\
\hline
\end{tabular}


In total, 85 continuous count sites and 1668 short-duration count sites have been surveyed in the past three years and were available for attribution.

As discussed in Section 3.3.1, continuous count sites take precedence over shortduration count sites, therefore for sequences with multiple count sites, the continuous count sites will be attributed to the sequence. Only 24 of the 382 multi-site sequences have continuous count sites, likely due to continuous count sites being less common than short-duration count sites and past efforts to minimize redundant data collection.

Table 7 outlines the distribution of count sites assigned to sequences by each principle after the first round of attribution. 
Table 7: Summary of count site attribution by principle after first round of sequencing and attribution

\begin{tabular}{|c|c|c|c|c|}
\hline \multicolumn{3}{|r|}{ Attribution Principle } & Number of & Total by \\
\hline 1 & a) & $\begin{array}{l}\text { Count sites are attributed to the sequences on } \\
\text { which they are situated. }\end{array}$ & 974 & 974 \\
\hline \multirow{6}{*}{2} & a) & $\begin{array}{l}\text { A site on an adjacent sequence may be } \\
\text { attributed to a sequence without a site if the } \\
\text { intersecting highway is a } 600 \text { series highway. }\end{array}$ & 238 & \multirow{6}{*}{469} \\
\hline & b) & $\begin{array}{l}\text { A count obtained on an adjacent sequence may } \\
\text { be attributed to a sequence without a site if the } \\
\text { intersecting highway has less than } 20 \% \text { of the } \\
\text { AADT on the adiacent sequence. }\end{array}$ & 116 & \\
\hline & c) & $\begin{array}{l}\text { A count site from an adjacent sequence may be } \\
\text { attributed to a sequence without a site if turning } \\
\text { movement counts show similar traffic patterns on } \\
\text { the two sequences. }\end{array}$ & 8 & \\
\hline & d) & $\begin{array}{l}\text { The count site of the adjacent sequence with the } \\
\text { highest AADT may be attributed to the centre } \\
\text { sequence of an offset intersection }\end{array}$ & 30 & \\
\hline & e) & $\begin{array}{l}\text { A sequence with a count site that has been } \\
\text { removed may be attributed volume from the } \\
\text { former count site with a growth factor applied. }\end{array}$ & 14 & \\
\hline & f) & $\begin{array}{l}\text { A count site from an adjacent sequence may be } \\
\text { attributed to a sequence if the two sequences } \\
\text { belong to different traffic control sections. }\end{array}$ & 63 & \\
\hline \multirow{4}{*}{3} & a) & $\begin{array}{l}\text { Continuous count sites take precedence over } \\
\text { short duration count sites. }\end{array}$ & 16 & \multirow{4}{*}{189} \\
\hline & b) & $\begin{array}{l}\text { The site with the most recent data takes } \\
\text { precedence over others. }\end{array}$ & 2 & \\
\hline & c) & $\begin{array}{l}\text { When AADTs } \leq 500: \text { if the count site with } \\
\text { greatest volume has an AADT less than twice } \\
\text { that of the lowest volume site, the highest } \\
\text { volume count site is attributed to the sequence }\end{array}$ & 119 & \\
\hline & d) & $\begin{array}{l}\text { When AADTs > 500: if the count sites have } \\
\text { AADTs that are less than } 20 \% \text { different from one } \\
\text { another, the highest volume count site is } \\
\text { attributed to the sequences }\end{array}$ & 52 & \\
\hline
\end{tabular}

157 of the 629 0:1 sequences and 1 many:1 sequence are attributed the default count site of the 2005 LRS or one based on engineering judgement. Additionally, 3 of the 629 $0: 1$ sequences are not attributed a count site or flow due to network discontinuity, for example, a gap introduced by a decommissioned bridge. 
1 of the 367 many: 1 sequences is attributed its default count site of the 2005 LRS, and 180 were not attributed count sites as a result of principle violations.

\subsection{EVALUATION OF SEQUENCING AND ATTRIBUTION}

The sequencing and attribution procedures yielded 1970 sequences (excluding 600 Series highways), 180 of which were not attributed count sites due to violations of the attribution principles. At this point, an additional 194 split locations were introduced, adding 194 sequences.

The completion of all methodology steps yielded a total of 2164 highway sequences, which is 118 more sequences than the 2046 sequences used in the previous linear referencing system of 2005. Figure 42 illustrates the sequenced highway network. 


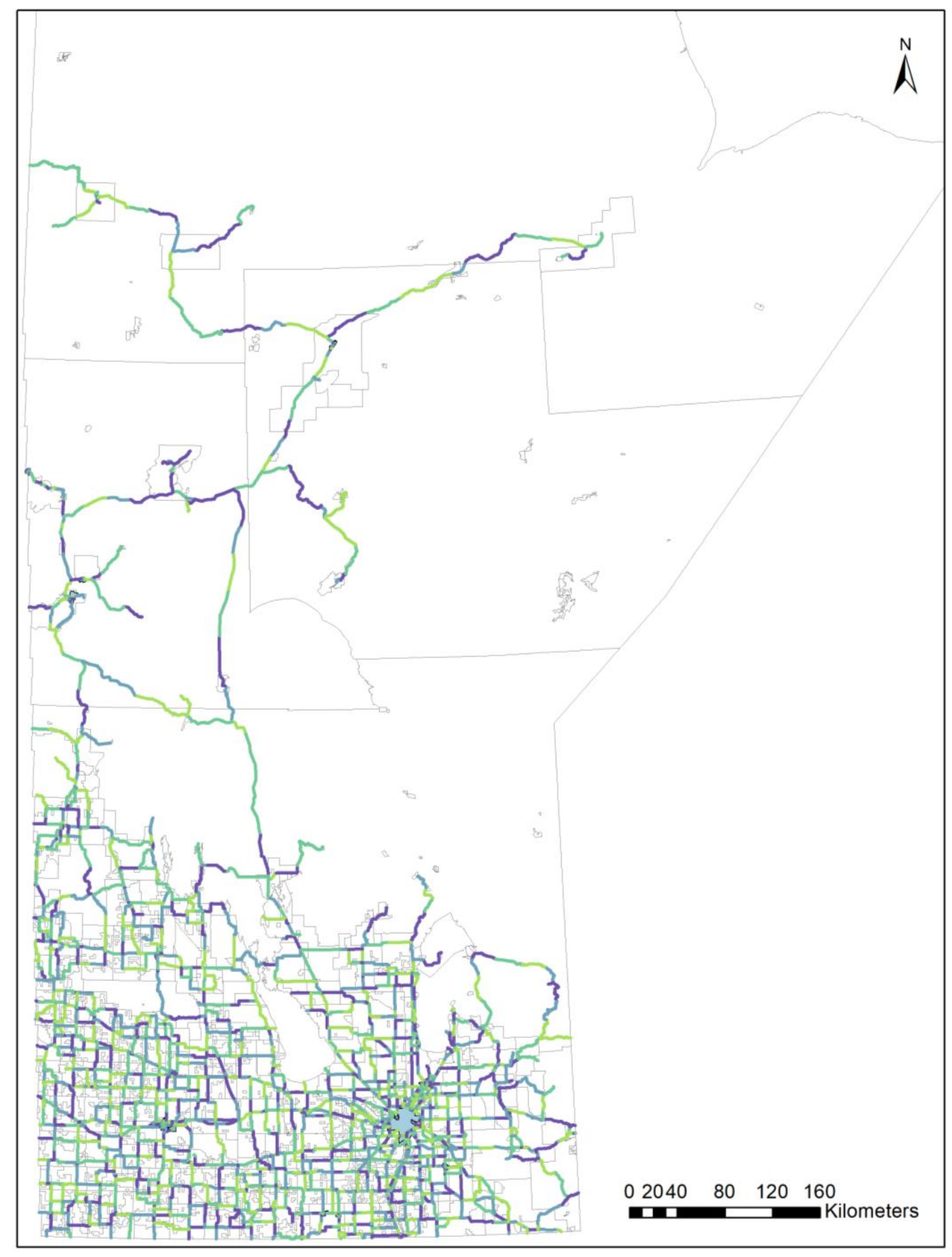

Figure 42: Final sequenced highway network

Table 8 summarizes sequences by highway type, and Table 9 summarizes sequences by length, described by the same length bins utilized in Table 3. 
Table 8: Number of sequences by highway type (excluding 600 Series highways)

\begin{tabular}{|l|l|}
\hline Highway Type & Number of Sequences \\
\hline PTH & 1067 \\
\hline PR & 1092 \\
\hline Other & 5 \\
\hline Total & 2164 \\
\hline
\end{tabular}

Table 9: Summary of sequences by length

\begin{tabular}{|l|l|l|l|l|}
\hline $\begin{array}{c}\text { Sequence } \\
\text { Lengths } \mathbf{( k m})\end{array}$ & $\begin{array}{c}\text { Sequence } \\
\text { Count }\end{array}$ & $\begin{array}{c}\text { Total Length } \\
\mathbf{( k m )}\end{array}$ & $\begin{array}{c}\text { \% of } \\
\text { Network }\end{array}$ & $\begin{array}{c}\text { \% of Network } \\
\text { Pre-Sequencing }\end{array}$ \\
\hline $0-9.99$ & 1533 & 6690.40 & 37.62 & 9.54 \\
\hline $10-19.99$ & 474 & 6499.51 & 36.54 & 31.43 \\
\hline $20-29.99$ & 105 & 2528.77 & 14.22 & 34.62 \\
\hline $30-39.99$ & 33 & 1108.92 & 6.23 & 14.56 \\
\hline $40-49.99$ & 11 & 486.33 & 2.73 & 6.58 \\
\hline $50-59.99$ & 6 & 327.25 & 1.84 & 2.45 \\
\hline $60-69.99$ & 1 & 67.20 & 0.38 & 0.38 \\
\hline $70-79.99$ & 1 & 77.60 & 0.44 & 0.44 \\
\hline Total & 2164 & 17785.98 & 100 & 100 \\
\hline
\end{tabular}

As expected, the number of highway sequences less than $10 \mathrm{~km}$ in length substantially increases, by more than $400 \%$, after the sequencing procedure. The number of sequences between 10 and $19.99 \mathrm{~km}$ in length also increases. All remaining length categories, barring the $60-69.99$ and $70-79.99$ categories that remain unchanged, decrease in count.

Of the 2164 highway sequences (excluding 600 Series highways), 629 (29.1\%) contained 0 count sites, 1337 (61.8\%) contained 1 count site, and 198 (9.1\%) contained more than 1 count site. The number of sequences with 1 count site increased from $49.3 \%$ to $61.9 \%$ of all sequences, whereas the number of sequences with 0 count sites decreased from $31.9 \%$ to $29 \%$ of all sequences and the number of sequences with more than 1 count site decreased from $18.7 \%$ to $9.1 \%$ of all sequences. These results were expected, as the goal of further sequencing was to allow for greater attribution. 
Count site attribution was performed upon completion of the final highway network sequencing. Table 10 outlines the distribution of count sites assigned to sequences by each principle.

Table 10: Summary of count site attribution by principle after completion of sequencing and attribution

\begin{tabular}{|c|c|c|c|c|}
\hline \multicolumn{3}{|r|}{ Attribution Principle } & Number of & Total by \\
\hline 1 & a) & $\begin{array}{l}\text { Count sites are attributed to the sequences on } \\
\text { which they are situated. }\end{array}$ & 1337 & 1337 \\
\hline \multirow{6}{*}{2} & a) & $\begin{array}{l}\text { A site on an adjacent sequence may be } \\
\text { attributed to a sequence without a site if the } \\
\text { intersecting highway is a } 600 \text { series highway. }\end{array}$ & 238 & \multirow{6}{*}{469} \\
\hline & b) & $\begin{array}{l}\text { A count obtained on an adjacent sequence may } \\
\text { be attributed to a sequence without a site if the } \\
\text { intersecting highway has less than } 20 \% \text { of the } \\
\text { AADT on the adjacent sequence. }\end{array}$ & 116 & \\
\hline & c) & $\begin{array}{l}\text { A count site from an adjacent sequence may be } \\
\text { attributed to a sequence without a site if turning } \\
\text { movement counts show similar traffic patterns on } \\
\text { the two sequences. }\end{array}$ & 8 & \\
\hline & d) & $\begin{array}{l}\text { The count site of the adjacent sequence with the } \\
\text { highest AADT may be attributed to the centre } \\
\text { sequence of an offset intersection }\end{array}$ & 30 & \\
\hline & e) & $\begin{array}{l}\text { A sequence with a count site that has been } \\
\text { removed may be attributed volume from the } \\
\text { former count site with a growth factor applied. }\end{array}$ & 14 & \\
\hline & f) & $\begin{array}{l}\text { A count site from an adjacent sequence may be } \\
\text { attributed to a sequence if the two sequences } \\
\text { belong to different traffic control sections. }\end{array}$ & 63 & \\
\hline \multirow{4}{*}{3} & a) & $\begin{array}{l}\text { Continuous count sites take precedence over } \\
\text { short duration count sites. }\end{array}$ & 16 & \multirow{4}{*}{343} \\
\hline & b) & $\begin{array}{l}\text { The site with the most recent data takes } \\
\text { precedence over others. }\end{array}$ & 2 & \\
\hline & c) & $\begin{array}{l}\text { When AADTs } \leq 500 \text { : if the count site with } \\
\text { greatest volume has an AADT less than twice } \\
\text { that of the lowest volume site, the highest } \\
\text { volume count site is attributed to the sequence }\end{array}$ & 125 & \\
\hline & d) & $\begin{array}{l}\text { When AADTs > 500: if the count sites have } \\
\text { AADTs that are less than } 20 \% \text { different from one } \\
\text { another, the highest volume count site is } \\
\text { attributed to the sequences }\end{array}$ & 54 & \\
\hline
\end{tabular}


188 sequences were flagged for improvement, including 13 sequences that were attributed traffic volume from past years with a growth factor applied, and 3 sequences that were not attributed count sites or traffic volume.

Figure 43 illustrates an assessment of the adequacy of count site to sequence attribution based on the number of count sites on the sequence and whether or not the sequences adhere to the attribution principles. Sequences that have a 1:1 count site-to-sequence ratio are assigned a "Good" rating. Sequences that have zero count sites and do not have major traffic sources or sinks at either end (or in the middle) are also assigned a "Good" rating as traffic volume from an adjacent sequence can reliably be attributed to the sequence with zero count sites. Sequences that have zero count sites and have nearby traffic sources or sinks, or are far from a data collection site, are assigned an "Inadequate" rating. The attributions in such cases may not provide an accurate representation of actual traffic volumes. Similarly, sequences within urban boundaries are assigned an "Inadequate" rating, due to the variability of traffic within a city/town. Finally, sequences with more than one count site are assigned an "Acceptable" rating, as the representation is reliable but there are redundant counts.

While the sequences with one count site are ideal and do not require modification, the sequences with zero count sites (and an "Inadequate" rating) or multiple count sites are candidates for improvements. Section 4.4 discusses this further. 


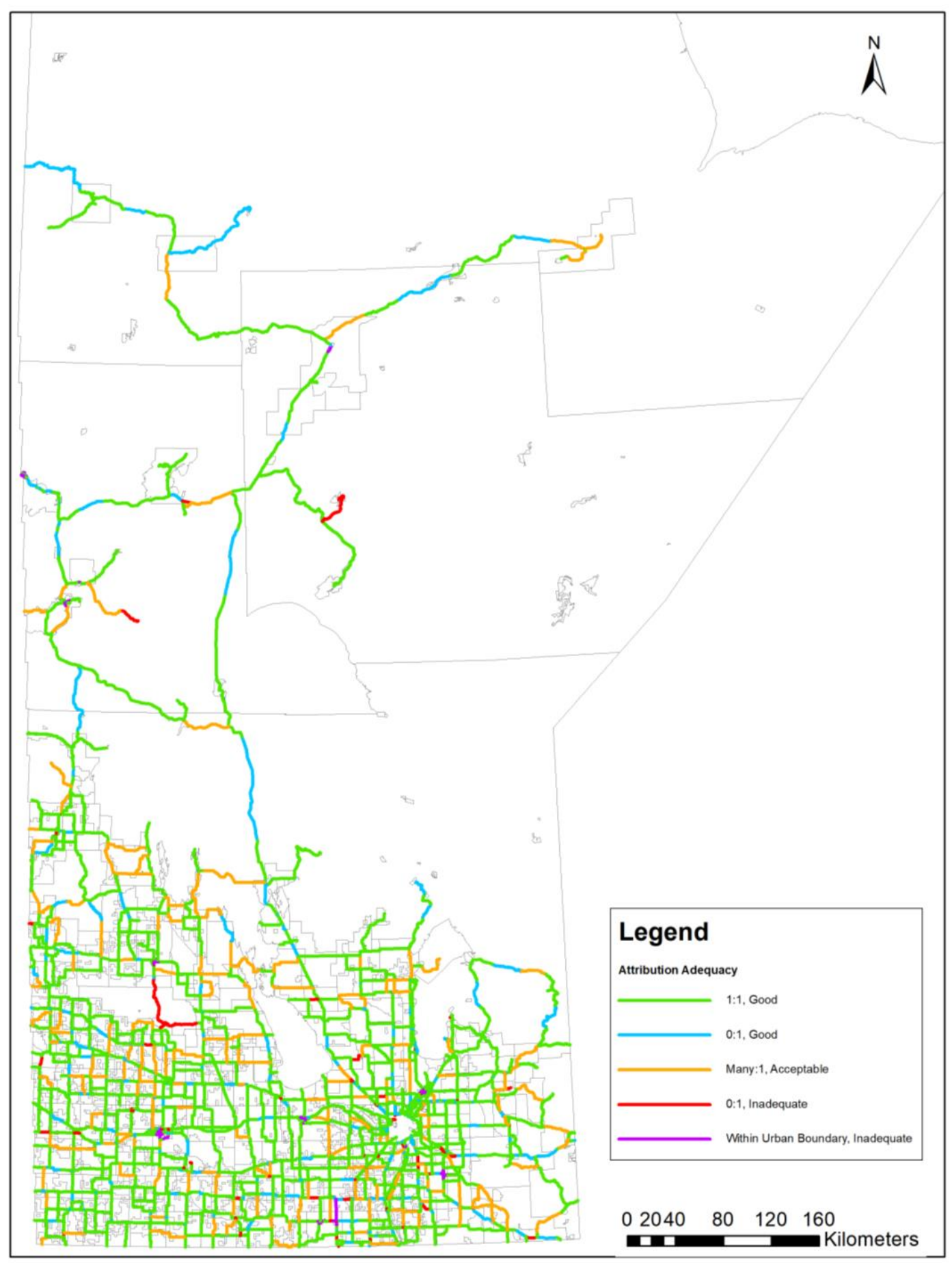

Figure 43: Highway sequences by attribution adequacy

Table 11 summarizes information from Figure 43, including the number of times each attribution rating is assigned and the total length of highway that is assigned each rating. 
Table 11: Summary of attribution rating by count and length

\begin{tabular}{|l|l|l|l|}
\hline \multicolumn{1}{|c|}{ Attribution Ratio and Rating } & \multicolumn{1}{c|}{ Count } & \multicolumn{1}{c|}{ Length (km) } & \% of Network \\
\hline $1: 1$, Good & 1319 & 11810.59 & 66.40 \\
\hline $0: 1$, Good & 466 & 1866.58 & 10.49 \\
\hline Many:1, Acceptable & 197 & 3453.99 & 19.42 \\
\hline $0: 1$, Inadequate & 77 & 526.07 & 2.96 \\
\hline Within Urban Boundary, Inadequate & 105 & 128.75 & 0.72 \\
\hline Total & 2164 & 17785.98 & 100.00 \\
\hline
\end{tabular}

The greatest number of sequences are attributed a "Good" rating, whereas the fewest are assigned an "Inadequate" rating, which is a desirable result for performance of the traffic monitoring program. While there are many opportunities for improvements in traffic data collection (as will be discussed further in Section 4.4), it should be noted that only $2.33 \%$ of the network by length has inadequate data collection based on the analysis of this research.

\subsection{OPPORTUNITIES TO IMPROVE HIGHWAY TRAFFIC DATA COLLECTION}

This section discusses the general issues identified throughout the sequencing, attribution and evaluation procedures. It also goes into further detail of specific issues encountered.

\subsubsection{General Issues}

The sequencing and attribution procedures provided insights into opportunities for improving data collection on Manitoba's highway network. This includes adding new count sites to critical locations and removing redundant count sites.

The evaluation identified 188 sequences where data collection sites can be improved and 203 count sites that are either redundant or within a city/town boundary. Appendix D gives the list of sequences, including the control section key, sequence number, highway number, concern, recommended action and priority for improvement. Appendix E gives 
the list of count sites, including the station number, highway number, concern and recommended action.

The key concerns for the 180 sequences follow:

1. The sequence lacks data collection. In such situations, the sequence either had a count site that has recently been removed or has never had a count site. This concern is separated into two tiers based on the significance of the sequence.

2. The sequence is within a city or town with a population of 5000 or greater. As previously discussed, traffic within cities/towns is highly variable and difficult to estimate with limited count sites, so sequences within city/town boundaries are flagged and a different method of attribution can be considered.

3. The sequence is problematic due to a change in the highway network. There are three locations in the province where bridges have been decommissioned, introducing gaps in the network that require accommodation in the traffic volume map.

The sequences are assigned a priority from 1 to 4 based on the degree of concern.

- Priority level 1 represents a significant lack of data collection, either on a sequence where data have never been collected, or on a key sequence where a site has been removed. Examples of this occur on the South and North Perimeter Highways, which is discussed further in Section 4.4.2. Twenty (20) sequences are assigned a level 1 priority.

- $\quad$ Priority level 2 is similar to priority level 1 , except that the sequences are less significant in the network (i.e., sequences that experience less traffic). The 
recommended action for these sequences is to install a count site on the sequence. In total, 89 sequences are assigned a level 2 priority.

- Priority level 3 sequences exist within a city/town. The recommendation to resolve this issue is to consider a new method of attributing traffic volume data to sequences within cities and towns. Sixty-eight (68) sequences are assigned a level 3 priority.

- Priority level 4 sequences have been impacted by a change in the highway network, such as a decommissioned bridge, and require an LRS update to remove the sequence. This occurs once in the highway network.

The evaluation also identified 203 count sites that are either redundant, such as when a sequence contains more than one count site, or are within city/town boundaries. As stated in Section 3.3.1, if a sequence has multiple count sites and the sites record traffic volumes that are either less than double one another (when AADT < 500) or less than $20 \%$ different (when AADT is 500 or greater), the highest AADT site is attributed to the sequence and the remaining site(s) is/are flagged. The evaluation identified 177 redundant count sites, which are recommended for removal. The resources used for the redundant count sites can be reallocated to fill data collection gaps in the future. The remaining 26 count sites are within city/town boundaries. Town Counts are performed separately from continuous and short-duration counts, and are used for different purposes. Processing of continuous and short-duration counts assumed that they represent uninterrupted traffic conditions; therefore, these sites should not be located inside urban boundaries. Count sites that are within urban boundaries should be redesignated to Town Counts, moved outside the city/town boundary, or removed. Sites that are within the city/town boundaries but outside the built up urban area of the city/town should be monitored for the city /town's future growth. The count sites can then 
be re-designated to Town Counts, moved outside the city/town boundaries, or removed as necessary. Figure 44 illustrates this concept.

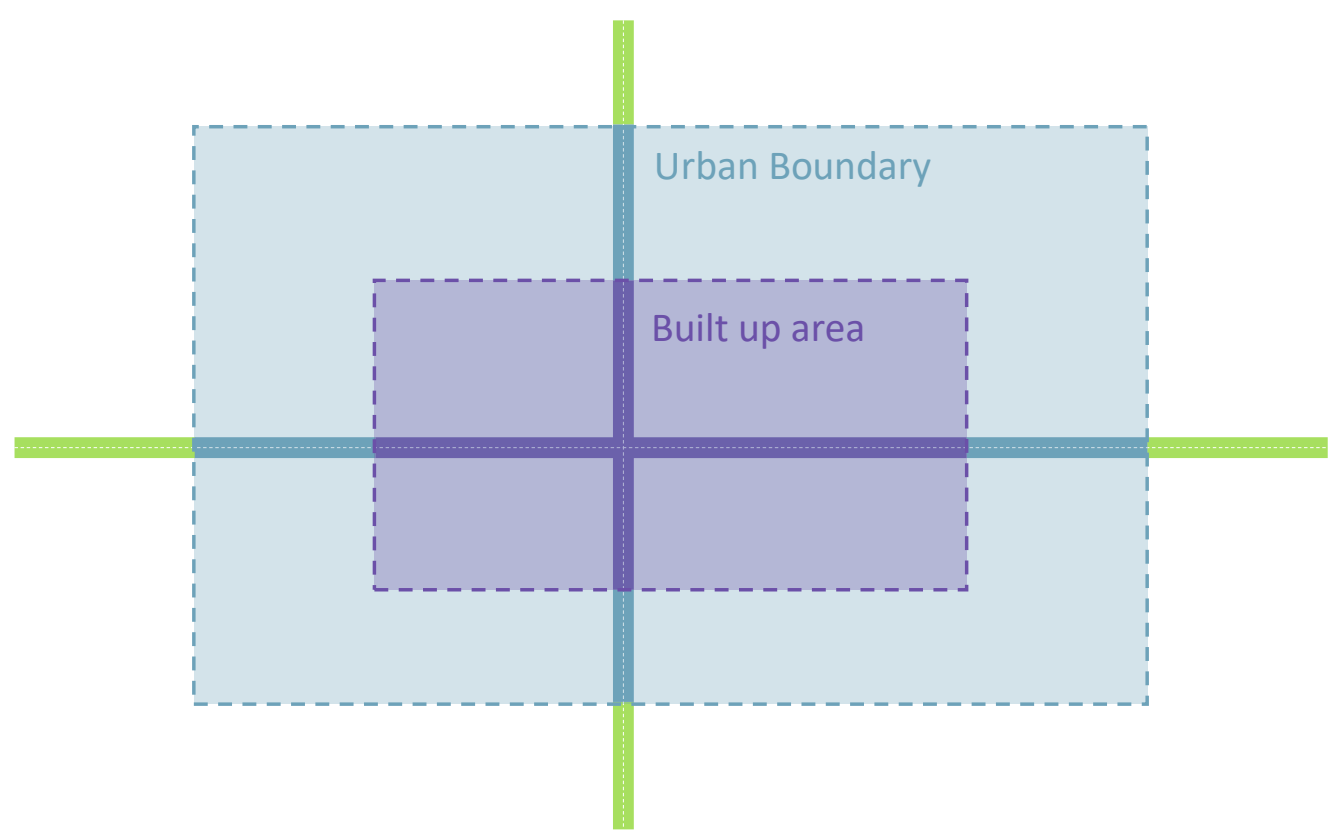

Figure 44: Illustration of built up area inside an urban boundary

\subsubsection{Specific Issues}

This section presents a more detailed analysis of specific sequence/count site concerns introduced in 4.4.1.

There are multiple instances where a new count site would be beneficial for obtaining network wide highway traffic statistics. The first of such locations is on sequences with high traffic volumes. There are three sequences on Winnipeg's Perimeter Highway that do not have count sites, yet the Perimeter Highway sees some of the greatest traffic volumes in the province. Consequently, these three sequences are assigned a priority level 1. Adding count sites to these sequences would allow for more accurate traffic volume estimates. Figure 45 highlights the three sequences on the perimeter highway that would benefit from additional count sites. 


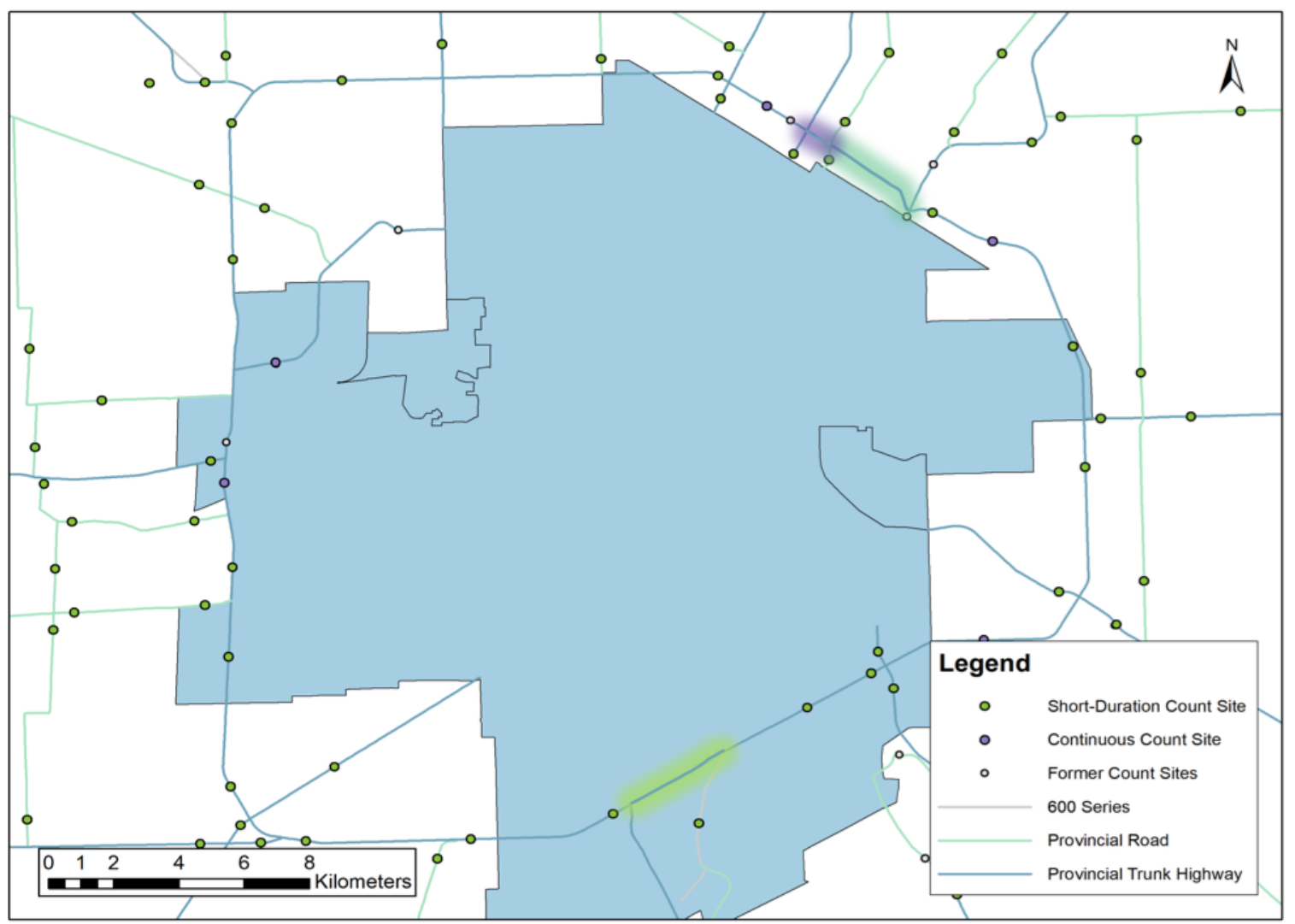

Figure 45: Highway sequences on the North and South Perimeter lacking data collection The two adjacent sequences on the North Perimeter and the sequence on the South Perimeter were previously attributed data from 2004 and 2005, respectively, which is the last year data were collected on either sequence. This was accounted for in attribution, where a count site was not attributed to the two sequences. Rather, traffic volume was attributed based on the 2004 count with a growth factor applied. The sequences were flagged as locations for potential traffic data collection upgrades.

A similar case occurs on PR 300 south of Winnipeg, where both count sites have been removed. Consequently, the sequences reference counts performed in the past, the most recent being in 2004. Similar to the previous case, the lack of data collection is accounted for in attribution, and growth factors are applied to past data to allow for traffic 
volume attribution of the sequence. Figure 46 illustrates the case. Similar to the case on the Perimeter Highway, the sequences were flagged for potential future upgrades.

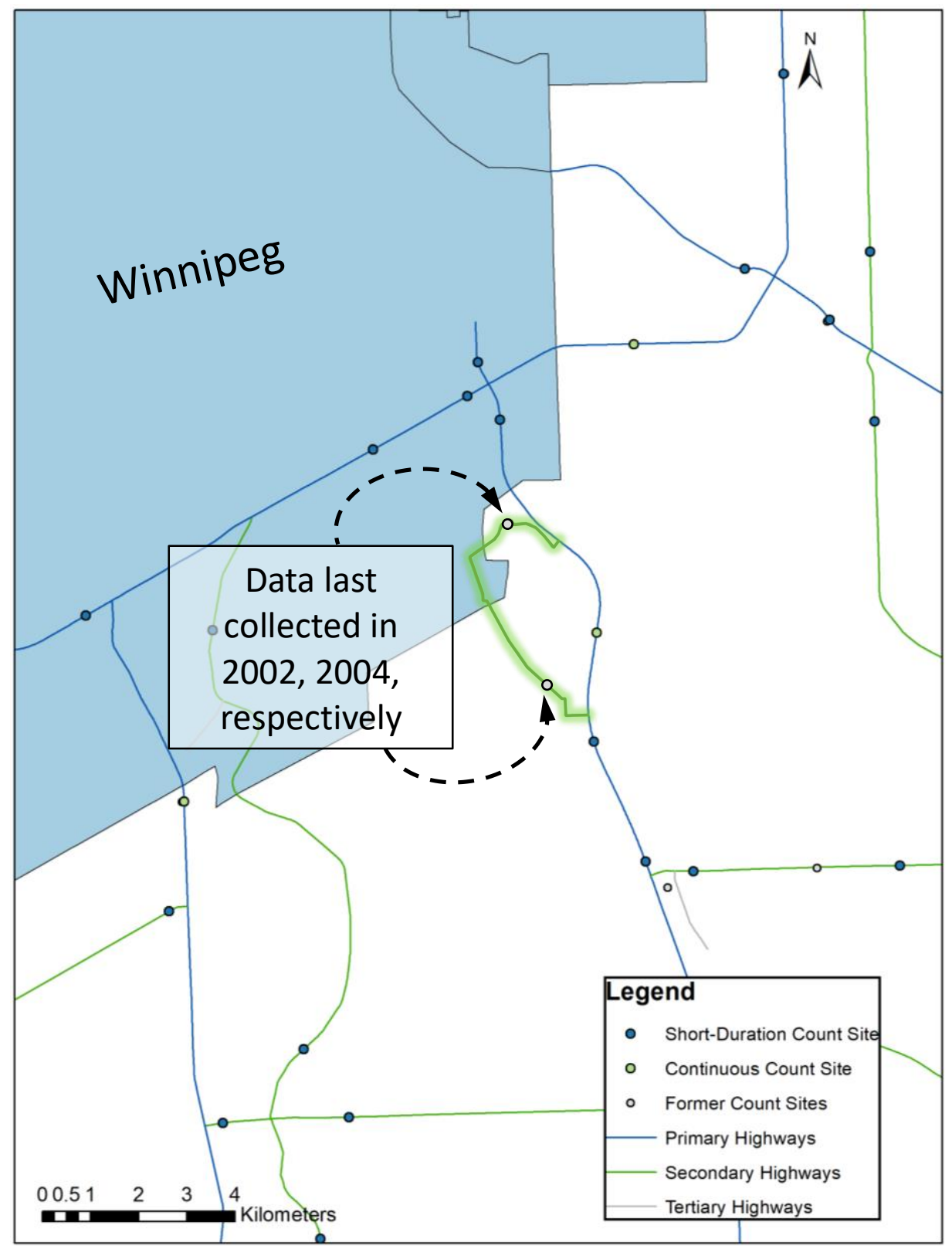

Figure 46: Sequences on PR 300 south of Winnipeg lacking data collection

A unique case that occurs in smaller towns throughout the province is the decommissioning of bridges. The resulting gap introduced into the network is often accompanied by a highway segment that has no traffic data. Figure 47 illustrates an 
example of this in Wawanesa, MB. Evidently, a monitoring program modification is required to ensure adequate network coverage. A similar example occurs in St. Jean Baptiste; however, the segment with no traffic count is very short $(0.15 \mathrm{~km})$.

Consequently, traffic data are not attributed to the segment.

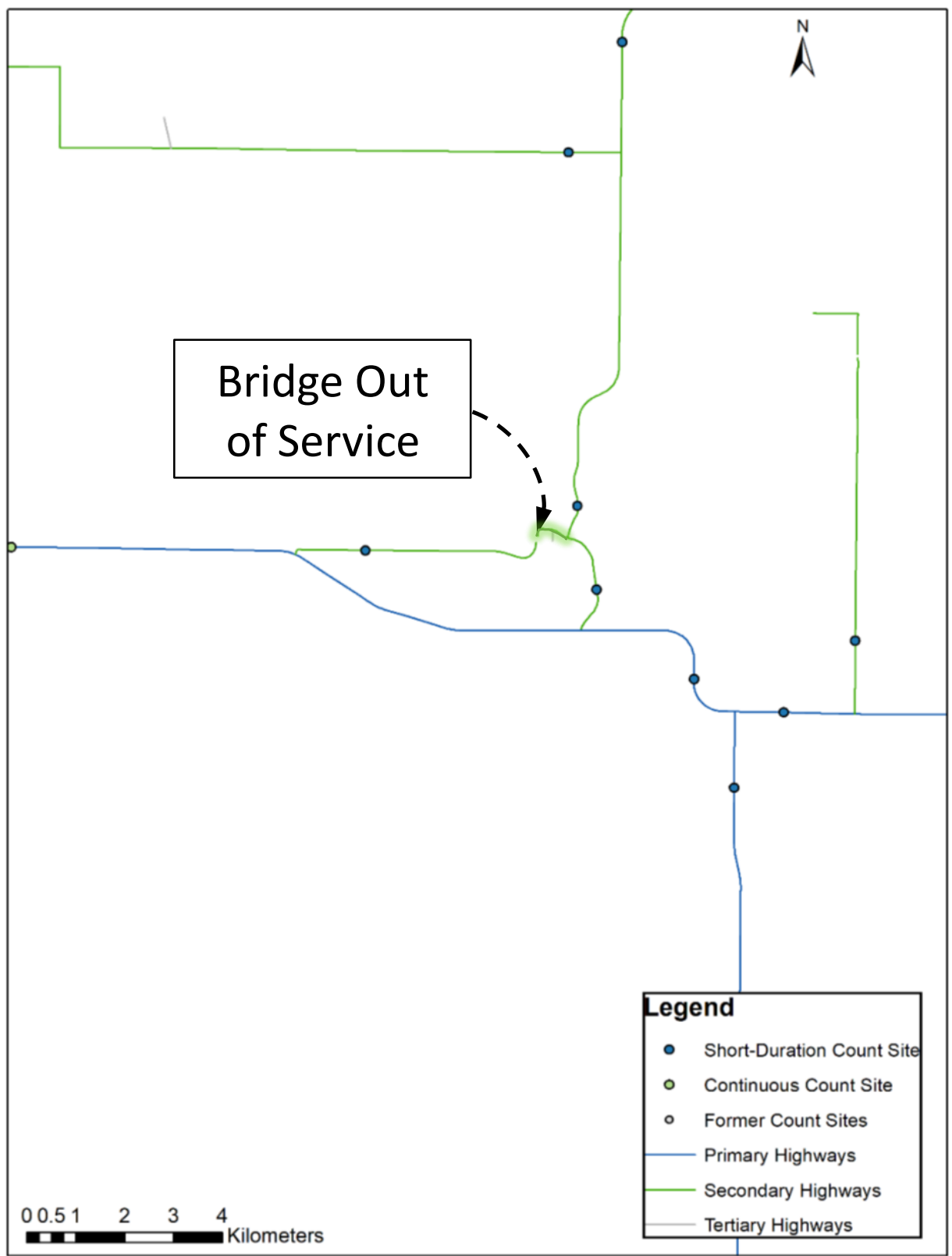

Figure 47: Bridge out of service near Wawanesa, MB, introducing a highway sequence with no traffic data 
Another unique case occurs in Ste. Anne, MB, where an entire segment of PR 207 has no past or present count sites. Consequently, the highway is attributed a site from a portion of PR 207 that is west of Ste. Anne. Figure 48 illustrates this case, with the highlighted segment being of key concern. This segment was attributed data as it was in the past; however, it was assigned a priority level 1 for future improvements.

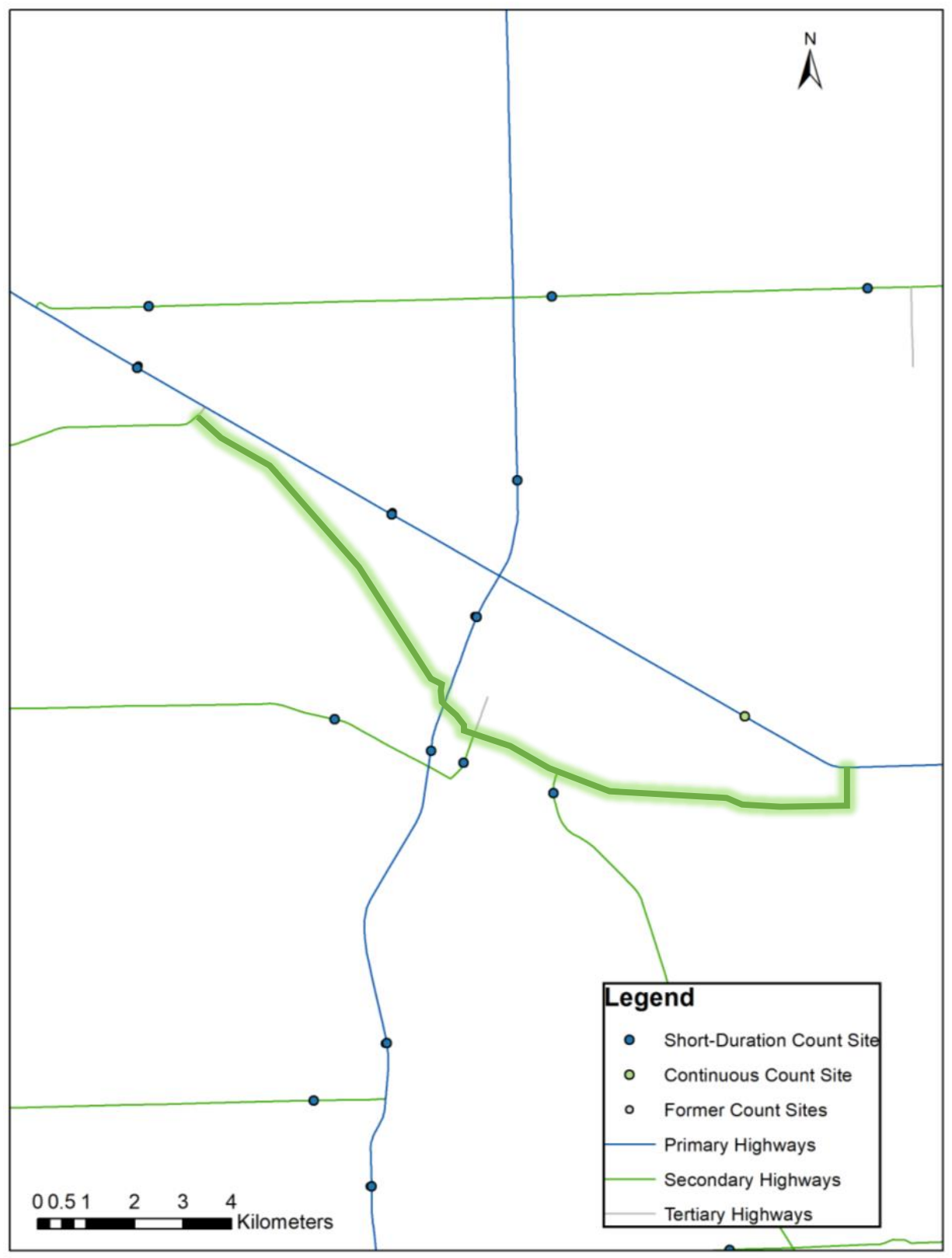

Figure 48: PR 207 sequences in Ste. Anne lacking data collection 


\subsection{SUMMARY AND FINAL RESULT}

This chapter summarized the results of the methodology, including both the sequencing and attribution procedures. It also discussed the evaluation of the two procedures, and the iteration of sequencing and attribution to produce the final volume map. Finally, the chapter outlined key findings of the research, including opportunities for improved data collection.

Figure 49 gives the final result of the methodology: a map of traffic volume on Manitoba's highways. Using the newly sequenced and attributed LRS produced by this research, the estimated 2017 VKT on Manitoba's provincial highway network is $8,034,247,090 \mathrm{~km}$. This is 0.5 percent higher than the VKT estimate of $7,991,225,700$ $\mathrm{km}$ produced using the previous LRS. The agreement between these values validates the sequencing and attribution procedures and reveals that meaningful changes were made. 


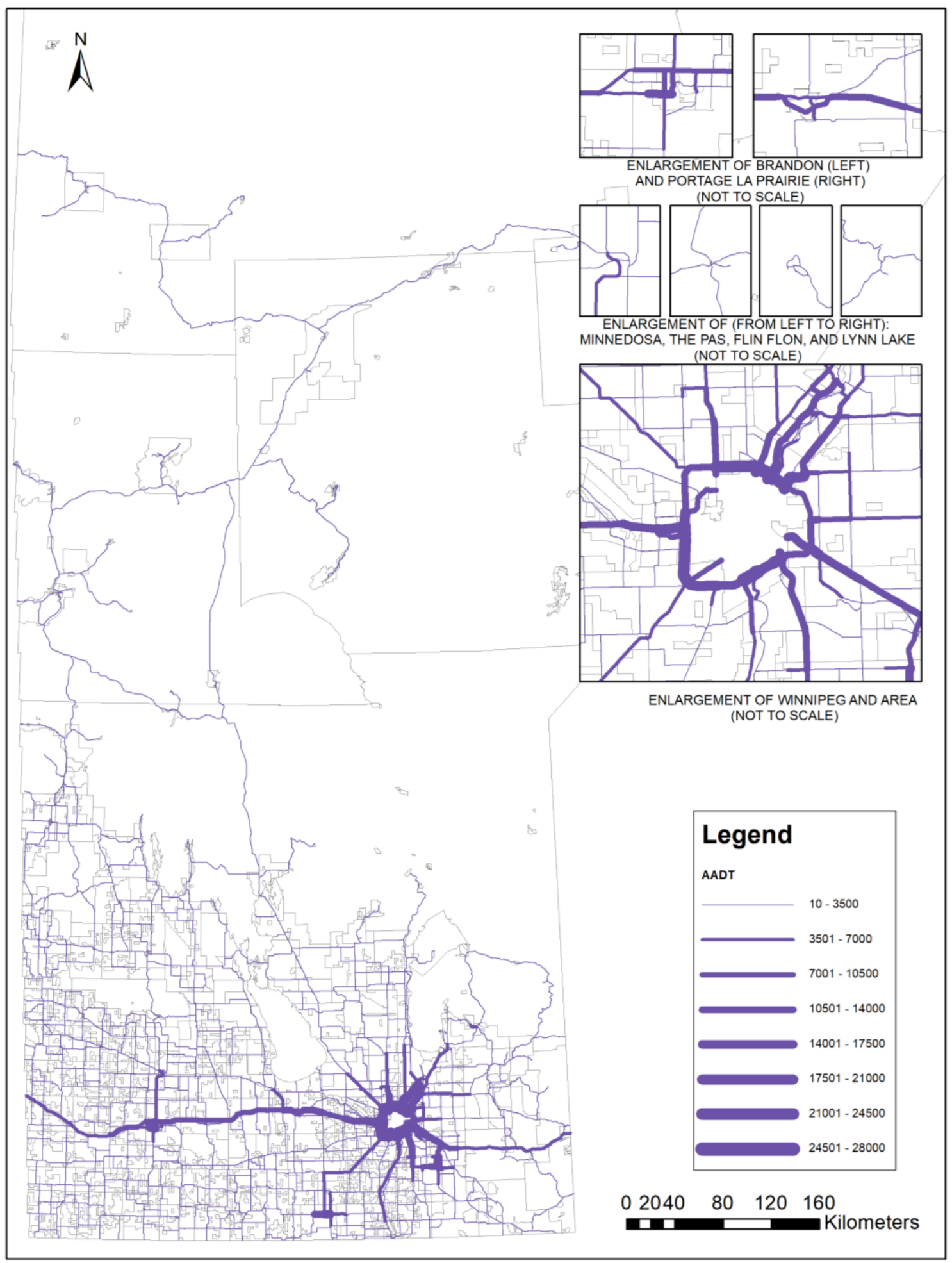

Figure 49: Manitoba highway traffic volume map 


\section{CONCLUSIONS}

This chapter highlights the key findings of this research and outlines opportunities for future work.

\subsection{SUMMARY OF KEY FINDINGS AND IMPLICATIONS}

This research creates a methodology for developing an LRS to manage and disseminate system-wide traffic volume data. The methodology comprises (1) a procedure to segment the highway network into sequences, and (2) a procedure to attribute count sites to sequences. The research applies the method in Manitoba.

The sequencing procedure involves deciding where highway segments shall be subdivided so that traffic volume is reasonably homogeneous along the length of each sequence (i.e., following the homogeneity assumption). Consequently, highways are sequenced at all PTH/PTH, PTH/PR, PTH/600 Series, PR/PR, PR/600 Series, and 600 Series/600 Series intersections, at intersections with major municipal roads, and at urban boundaries. The sequencing procedure also involves numerating sequences so that each sequence has a unique combined control section key and sequence. Application of this procedure created 1970 sequences excluding 600 Series highways, or 2365 sequences including 600 Series highways.

The attribution procedure involves developing principles for attributing count sites to highway sequences. The principles consider the strength of the data collected (i.e., whether the station collects data permanently or for a short-duration during the year), the recency of the data collected, and the influences of other highways or traffic sources/sinks on the traffic volume of a given sequence. Considering these key elements, this research develops three multipart principles for count site attribution: principle 1 for sequences with one count site, principle 2 for sequences with zero count 
sites and principle 3 for sequences with more than one count site. This procedure attributed count sites to 1790 of 1970 sequences. The remaining 180 sequences required evaluation and further sequencing to allow for attribution.

After evaluation of the first round of sequencing and attribution, the research utilizes engineering judgement to further sequence the network where sequences have multiple count sites with significantly different data. Sequences with multiple count sites where data are significantly different (where the greater volume site's AADT is less than double the lesser volume's site when AADT $\leq 500$, or $>20 \%$ difference when AADT is $>500$ ) do not meet the homogeneity assumption and therefore further sequencing is necessary. Locations of additional sequencing include highway vertices, major origins/destinations, changes in road surface type, and at cities/towns or roads leading to cities/towns. This procedure added an additional 194 sequencing locations, yielding a total of 2164 sequences.

This research also highlights sequences that lack appropriate data collection, as well as count sites that are redundant or require moving. It then summarizes these locations based on perceived importance for future improvement. Of the 2164 sequences, 188 are highlighted for data collection improvements, and of the 1753 count sites, 203 are highlighted for re-designating, moving, or removal.

Traffic monitoring is vital in producing traffic statistics used for designing roadways, quantifying safety, and improving pavement design, among other functions. To make the greatest benefit in these areas, traffic monitoring data must be as accurate and easily accessible as possible. This research benefits traffic monitoring in that it provides a methodology to represent real-life traffic volumes accurately in a way that is accessible by professionals and the general public. It also suggests future improvements for 
Manitoba's traffic monitoring program, including where count sites should be added and removed.

Beyond the findings specific to Manitoba, this research also reveals broader issues relevant to traffic monitoring professionals in any jurisdiction:

- Methods of segmenting a highway network and attributing data to highway segments are not well documented by jurisdictions. While it is evident that network segmentation occurs, as this is necessary to attribute data to segments, the methods by which jurisdictions perform this task are unclear. This highlights a gap in knowledge and a need for this research.

- The variability of transportation networks in the real world introduce unique situations in sequencing segments and attributing count sites. This creates complexities in developing criteria and principles for these procedures, as it is difficult to encompass all cases. Consequently, some engineering judgement is necessary to complete the procedures and achieve the goals of the research. Locations that still create issues should be considered for future improvements or upgrades that will allow the traffic monitoring program to work more efficiently and effectively.

- The methodologies developed in this research allow for an evaluation of the traffic monitoring program and identifying opportunities for improved data collection, be it by obtaining greater coverage or eliminating redundancies. Jurisdictions, including Manitoba, would benefit from introducing count sites on highways with limited data collection, particularly those that see high volumes of traffic. Similarly, jurisdictions would benefit from removing redundant counts, as this would allow reallocation of resources to obtain greater network coverage and further improve the traffic monitoring program. 
- Changes to the highway network (e.g., highway twinning, realignment, bridge removal) require an evaluation of the traffic monitoring program to ensure there is adequate network coverage. Regular evaluation of highway network changes will ensure the linear referencing system is up to date and providing the most representative depiction of real-life traffic volumes possible.

- From the perspective of accurately representing traffic activity on a network, it is beneficial to attain a 1:1 sequence-to-count site ratio. However, this may be impractical to achieve. Consequently, there is an opportunity to explore other methods of sampling, specifically hierarchical sampling.

\subsection{OPPORTUNITIES FOR FUTURE RESEARCH AND WORK IN TRAFFIC MONITORING}

This research has revealed the following future research needs:

- With continuous count sites collecting data 24 hours per day for 365 days of the year, they are known to produce reliable data for the highway sequences on which they are situated, particularly in comparison with short-duration count sites. There is an opportunity for further research to determine if a hierarchical attribution, one where a continuous count site on an adjacent sequence would take precedence over a short-duration count site on the sequence of interest, to determine if this would produce more representative traffic data statistics and traffic volume maps.

- At present, Manitoba collects turning movement counts, which are accessible on the MHTIS website, however does not use these counts in producing traffic statistics. In Manitoba, typically at least 48 hours of data are required to produce an AADT estimate. While not typical, some turning movement counts are 48 hours or greater in duration, which have the potential to produce an AADT estimate for the highway sequences at the intersection. Research into determining the feasibility of utilizing 
turning movement counts to produce highway network traffic statistics would have the potential to benefit traffic monitoring in Manitoba and elsewhere.

- This research introduces a method of sequencing the highway network and attributing count sites to sequences. However, it does not discuss a method of updating the LRS from year to year to prevent repetition of this process.

Consequently, determining a method to update the LRS to reflect highway geometry changes that occur throughout the year is an opportunity for future work.

- While Manitoba does not presently have a monitoring program for 600 Series highways, this is an option for the future, as this research has considered 600 Series in sequencing the network. The LRS developed in this research is adaptable to future data collection on 600 Series highways if the province chooses to do so.

- Currently, the AADT estimates for highway segments within towns come from continuous and short-duration counts, which typically exist outside of town boundaries. Future research may investigate the opportunity for a traffic monitoring program to utilize town counts to provide more accurate AADT estimates of highways within towns.

\subsection{CONCLUSION}

This research develops four criteria for segmenting the highway network into sequences based on locations of traffic sources and sinks, such as highway intersections or urban areas. It also develops three multipart principles for attributing traffic data to highway sequences, considering the type of count site, the proximity of the site to the sequence, the recency of data collection, and the presence of traffic sources and sinks. The application of the sequencing and attribution procedures enables practitioners to improve the spatial representativeness of a traffic volume map and reveals the importance of evaluating the traffic monitoring program when changes are made to the highway network or sampling program. 


\section{BIBLIOGRAPHY}

Alberta Ministry of Transportation. (2017). Traffic Counts. Retrieved January 4, 2018, from Alberta Transportation: http://www.transportation.alberta.ca/mapping/

Alberta Ministry of Transportation. (2017). Traffic Volumes at Points on the Highway Network. Retrieved 4 2017, January, from Alberta Transportation: http://www.transportation. alberta.ca/2639.htm

American Association of State Highway and Transportation Officials. (2009). AASHTO Guidelines for Traffic Data Programs. Washington, D.C.: AASHTO.

Applied Engineering Management Corporation. (2018). NCHRP Research Report 865: Guidance for Development and Management of Sustainable Enterprise Information Portals. Washington, D.C.: Transportation Research Board of the National Academies.

BC Ministry of Transportation and Infrastructure. (2017). Traffic Data Program GIS Application. Retrieved January 4, 2018, from https://prdoas3.pubapps.th.gov.bc.ca/tsg/

Berté, W. K., de Souza, D. V., \& Leitão, L. J. (2016). Geographic Information System as a Supportive Tool for Road Accident Investigation. Proceedings from the 17th Internation Conference Road Safety on Five Continents (RS5C). Rio de Janeiro, Brazil: Road Safety on Five Continents (RS5C).

Bingham, J., \& Kang, S. (2013). Building a Highway Linear Referencing System from Preexisting Reference Maker Measurements for Transportation Data Management. Safety Transportation Research \& Education Center.

Burdett, A. (2010). Electronic Handover of 'As-Constructed Asset Data' - A Real World Example. CORE 2010, Rail - Rejuvenation and Renaissance, Conference on Railwaly Engineering (pp. 365-375). Wellington, New Zealand: Railway Technical Society of Australasia.

Colorado Department of Transportation. (2017). Traffic Volume on Colorado Highways. Retrieved from Colorado Department of Transportation:

http://cdot. $m a p s$. arcgis.com/apps/webappviewer/index.html?id=483fb9a9e86a4b 238d1deec6dcd60a77

ESRI. (2016). What is Linear Referencing? Retrieved 01 22, 2017, from Environmental Systems Research Institute: http://desktop.arcgis.com/en/arcmap/10.3/guidebooks/linear-referencing/what-is-linear-referencing.htm

Federal Highway Administration. (2016). Traffic Monitoring Guide. Washington, D.C.: U.S. Department of Transportation.

Gharaibeh, N., Oti, I., Schrank, D., \& Zmud, J. (2017). Data Management and Governance Practices: A Synthesis of Highway Practice. Washington, D.C.: Transportation Research Board of the National Academies. 
Goldwyn, E., \& Vergel-Tovar, C. (2018). Mapping the unmapped transit network of Bogota, Colombia. Washington, D.C.: Transportation Research Board of the National Academies.

Highway Planning \& Design Branch. (2005). Provincial Highway Referencing System Definitions \& Descriptions Manual (Draft). Winnipeg, MB: Manitoba Transportation \& Government Services.

Ma, X., \& Wang, Y. (2014). Development of a Data-Driven Platform for Transit Perforamnce Measures Using Smart Card and GPS Data. Journal of Transportation Engineering, 140(12).

Manitoba Highway Traffic Information System. (2017). MHTIS Webmap. Retrieved from MHTIS: http://umtig.eng.umanitoba.ca/mhtis_traffic_2016_2/framesetup.asp

Mekuria, M. C., \& Seedah, D. P. (2018, February 27). TRB Webinar: Spatial Modeling for Highway Performance Monitoring System Data.

Minnesota Department of Transportation. (2017). MnDOT Traffic Data. (Minnesota Department of Transportation) Retrieved January 4, 2018, from MnDOT GIS: http://mndotgis.dot.state.mn.us/tfa/Map

Nardini, P. D., Zarghamee, M. S., Bain, L., Jalbert, C., \& Remer, A. (2015). Incorporating GIS-Based Structural Evaluation Tools into Pipeline Asset Management. Pipelines 2015, 635-645.

North Carolina Department of Transportation. (2016). NCDOT AADT Web Map. (North Carolina Department of Transportation) Retrieved January 4, 2018, from NCDOT GIS Online:

http://ncdot.maps.arcgis.com/home/webmap/viewer.html?webmap=b7a26d6d8ab d419f8c27f58a607b25a1

North Carolina Department of Transportation Traffic Survey Group. (2016). NCDOT 2016 AADT Stations Shapefile. Retrieved January 4, 2018, from Connect NCDOT: https://connect.ncdot.gov/resources/StateMapping/Documents/2016_NCDOT_AADT_Stations_Shapefile_Description.pdf

North Carolina Department of Transportation Traffic Survey Group. (2016). NCDOT 2016 Traffic Data Segment Shapefile. Retrieved January 4, 2018, from Connect NCDOT: https://connect.ncdot.gov/resources/StateMapping/Documents/NCDOT_2016_Traffic_Segment_Shapefile_Description.pdf

Nova Scotia Transportation and Infrastructure Renewal Department. (2017, April 5). Traffic Volumes - Provincial Highway System. Retrieved January 4, 2018, from https://data.novascotia.ca/Roads-Driving-and-Transport/Traffic-VolumesProvincial-Highway-System/8524-ec3n/data

Olsen, M. J., Raugust, J. D., \& Roe, G. V. (2013). NCHRP Synthesis 446: Use of Advanced Geospatial Data, Tools, Technologies, and Information in Department of Transportation Projects. Washington, D.C.: Transportation Research Board of the Natioanl Academies. 
Ontario Ministry of Transportation. (2010). iCorridor. (Ontario Ministry of Transportation) Retrieved January 4, 2018, from http://www.maps.mto.gov.on.ca/icorridor/WebPages/Map/FundyViewer.aspx

Ontario Ministry of Transportation. (2016, December 8). Ontario Provincial Highways Traffic Volumes on Demand. (Government of Ontario) Retrieved January 5, 2018, from Ontario Ministry of Transportation: http://www.raqsa.mto.gov.on.ca/techpubs/TrafficVolumes.nsf/tvweb

Regehr, J. D., \& Reimer, M. J. (2013). Methodology to Estimate the Distance Traveled by Trucks on Rural Highway Systems. Journal of Transportation Engineering, 390-398.

Regehr, J. D., Poapst, R., Rempel, G., Montufar, J., \& Hallenbeck, M. (2017). Traffic Monitoring Practices Guide for Canadian Provinces and Municipalities. Ottawa, ON: Transportation Association of Canada.

Rempel, G., Regehr, J., \& Montufar, J. (2013). Principles for addressing urban traffic monitoring challenges. Winnipeg: Transportation Association of Canada.

Rouzbeh, S., \& Shahriar, R. (2017). Identification of Road Crash Black-Sites Using Geographical Information System. International Gournal for Traffic and Transport Engineering, 7(3), 368-380.

Saskatchewan Ministry of Highways \& Infrastructure. (2017). 2016 Traffic Volume Map Average Annual Daily Traffic. Retrieved from Government of Saskatchewan: http://publications.gov.sk.ca/documents/18/1001992016_Highway_Traffic_Volume_Maps.pdf

Shuna, X., \& Changpo, L. (2012). Construction of Urban Bus System Database Using Dynamic Segmentation Techniques - a case study of Xuchang City. IEEE.

Statistics Canada. (2018, February 8). Population and Dwelling Count Highlight Tables, 2016 Census. Retrieved August 28, 2018, from Statistics Canada:

https://www12.statcan.gc.ca/census-recensement/2016/dp-pd/hlt-fst/pd-

$\mathrm{pl} /$ Table.cfm? Lang=Eng\&T=302\&SR $=1 \& \mathrm{~S}=58 \& \mathrm{O}=\mathrm{A} \& \mathrm{RPP}=25 \& \mathrm{PR}=46 \& \mathrm{CMA}=0 \#$ tPopDwell

Stolz, L. (2017). Continuous-Count Traffic Programs. In Advancing Highway Traffic Monitoring Through Strategic Research (pp. 13-17). Washington, D.C.: Transportation Research Board of the National Academies.

Stull, M. M., Jackson, K. N., \& O'Brien, S. W. (2017). Large-Scale Collection and Formatting of Pedestrian and Bicycle Geospatial Assets in North Carolina. Washington, D.C.: Transportation Research Board of the National Academies. 


\section{APPENDIX A: FHWA 15-VEHICLE CLASSIFICATION SCHEME}

\begin{tabular}{|l|l|}
\hline & FHWA Class 1 - Motorcycles \\
\hline & $\begin{array}{l}\text { FHWA Class 2 - Passenger Vehicles (With 1- } \\
\text { or 2-Axle Trailers) }\end{array}$ \\
\hline & $\begin{array}{l}\text { FHWA Class 3 - 2-Axles, 4-Tire Single Units, } \\
\text { Pickup or Van (With 1 - or 2-Axle Trailers) }\end{array}$ \\
\hline
\end{tabular}




\begin{tabular}{|l|l|l|}
\hline $0=0000$ & $\begin{array}{l}\text { FHWA Class } 15-11 \text { or More Axles, Multi- } \\
\text { Trailers }\end{array}$ \\
\hline
\end{tabular}




\title{
APPENDIX B: SHAPEFILE METADATA
}

\section{DATA FRAME COORDINATE SYSTEM}

\author{
NAD 1983 UTM Zone 14N \\ WKID: 26914, Authority: EPSG \\ Projection: Transverse Mercator \\ False Easting: 500000.0 \\ False Northing: 0.0 \\ Central Meridian: -99.0 \\ Scale Factor: 0.9996 \\ Latitude of Origin: 0.0 \\ Linear Unit: Meter (1.0) \\ Geographic Coordinate System: GCS North American 1983 \\ Angular Unit: Degree (0.0174532925199433) \\ Prime Meridian: Greenwich (0.0) \\ Datum: North American Datum of 1983 \\ Spheroid: Geodetic Reference System 1980 \\ Semi-major Axis: 6378137.0 \\ Semi-minor Axis: 6356752.314140356 \\ Inverse Flattening: 298.257222101
}

\section{FEATURE CLASS DATA}

\section{LRS_HIGHWAY_NETWORK_2015}

Source: Manitoba Infrastructure

Data Type: File Geodatabase Feature Class

Feature Class: LRS_HIGHWAY_NETWORK_2015

Feature Type: Simple

Geometry Type: Line

Coordinates have $Z$ values: No

Coordinates have measures: No

\section{Manitoba_Subdivisions}

Source: Natural Resources Canada

Data Type: File Geodatabase Feature Class

Feature Class: Manitoba_Subdivisions

Feature Type: Simple

Geometry Type: Polygon

Coordinates have $Z$ values: No

Coordinates have measures: No 


\section{LRS_2005}

Source: Manitoba Infrastructure

Data Type: File Geodatabase Feature Class

Feature Class: LRS_2005

Feature Type: Simple

Geometry Type: Line

Coordinates have $Z$ values: No

Coordinates have measures: No

\section{Station}

Source: Manitoba Highway Traffic Information System (MHTIS)

Data Type: File Geodatabase Feature Class

Feature Class:Station

Feature Type: Simple

Geometry Type: Multipoint

Coordinates have $Z$ values: No

Coordinates have measures: No 


\title{
APPENDIX C: PYTHON CODE FOR FIELD CALCULATIONS
}

\author{
START KM FIELD CALCULATOR \\ Pre-Logic Script Code: \\ Start $=0$ \\ LastEndKM $=0$ \\ def StartKM(SeqNumber, LengthKM): \\ global Start \\ global LastEndKM \\ if $($ SeqNumber $==10)$ : \\ Start $=0$ \\ else: \\ Start $=$ LastEndKM \\ LastEndKM = LastEndKM + LengthKM \\ return Start
}

[Field $]=$

StartKM(!SEQUENCE_NO!, !LENGTH_KM!) 


\section{APPENDIX D: SEQUENCES FOR IMPROVED TRAFFIC MONITORING}

\begin{tabular}{|c|c|c|c|c|c|}
\hline CS Key & Sequence & Highway & Concern & Recommendation & Priority \\
\hline 01007010HA & 10 & 7 & Significant intersecting highway AADT & Install count site & 2 \\
\hline 01009060HU & 10 & 9 & Significant intersecting highway AADT & Install count site & 2 \\
\hline 01009060HU & 20 & 9 & No current data collection & Install count site & 2 \\
\hline 01009060HU & 30 & 9 & Within city/town boundary (Selkirk) & $\begin{array}{l}\text { Consider different method of count site } \\
\text { attribution }\end{array}$ & 3 \\
\hline 01009910HA & 10 & 9 & No current data collection & Install count site & 2 \\
\hline 01009910HA & 20 & 9 & Within city/town boundary (Selkirk) & $\begin{array}{l}\text { Consider different method of count site } \\
\text { attribution }\end{array}$ & 3 \\
\hline 01009910HA & 30 & 9 & Within city/town boundary (Selkirk) & $\begin{array}{l}\text { Consider different method of count site } \\
\text { attribution }\end{array}$ & 3 \\
\hline 01009915HU & 10 & 9 & Within city/town boundary (Selkirk) & $\begin{array}{l}\text { Consider different method of count site } \\
\text { attribution }\end{array}$ & 3 \\
\hline $01011060 \mathrm{HU}$ & 30 & 11 & Significant intersecting highway AADT & Install count site & 2 \\
\hline $01011060 \mathrm{HU}$ & 40 & 11 & Significant intersecting highway AADT & Install count site & 2 \\
\hline 01012020HU & 10 & 12 & Significant intersecting highway AADT & Install count site & 2 \\
\hline $01012050 \mathrm{HU}$ & 40 & 12 & Within city/town boundary (Steinbach) & $\begin{array}{l}\text { Consider different method of count site } \\
\text { attribution }\end{array}$ & 3 \\
\hline $01012055 \mathrm{HA}$ & 10 & 12 & Within city/town boundary (Steinbach) & $\begin{array}{l}\text { Consider different method of count site } \\
\text { attribution }\end{array}$ & 3 \\
\hline 01012060HA & 10 & 12 & Within city/town boundary (Steinbach) & $\begin{array}{l}\text { Consider different method of count site } \\
\text { attribution }\end{array}$ & 3 \\
\hline $01015030 \mathrm{HU}$ & 20 & 15 & Significant intersecting highway AADT & Install count site & 2 \\
\hline $01015050 \mathrm{HU}$ & 30 & 15 & Significant intersecting highway AADT & Install count site & 2 \\
\hline $01023130 \mathrm{HU}$ & 10 & 23 & Significant intersecting highway AADT & Install count site & 2 \\
\hline $01044020 \mathrm{HU}$ & 10 & 44 & Significant intersecting highway AADT & Install count site & 2 \\
\hline 01044040HU & 10 & 44 & Significant intersecting highway AADT & Install count site & 2 \\
\hline 01052010HU & 30 & 52 & Significant intersecting highway AADT & Install count site & 2 \\
\hline 01052015HA & 20 & 52 & Within city/town boundary (Steinbach) & $\begin{array}{l}\text { Consider different method of count site } \\
\text { attribution }\end{array}$ & 3 \\
\hline
\end{tabular}




\begin{tabular}{|c|c|c|c|c|c|}
\hline CS Key & Sequence & Highway & Concern & Recommendation & Priority \\
\hline 01052020HA & 10 & 52 & Within city/town boundary (Steinbach) & $\begin{array}{l}\text { Consider different method of count site } \\
\text { attribution }\end{array}$ & 3 \\
\hline $01059020 \mathrm{HU}$ & 40 & 59 & Significant intersecting highway AADT & Install count site & 2 \\
\hline 01059040HU & 20 & 59 & Significant intersecting highway AADT & Install count site & 2 \\
\hline $01059040 \mathrm{HU}$ & 30 & 59 & Significant intersecting highway AADT & Install count site & 2 \\
\hline 01059045HA & 10 & 59 & Significant intersecting highway AADT & Install count site & 2 \\
\hline 01059070HA & 10 & 59 & No current data collection & Install count site & 1 \\
\hline 01059080HA & 10 & 59 & No current data collection & Install count site & 1 \\
\hline $01059140 \mathrm{HU}$ & 20 & 59 & Significant intersecting highway AADT & Install count site & 2 \\
\hline $010750800 A$ & 10 & 75 & Within city/town boundary (Winnipeg) & $\begin{array}{l}\text { Consider different method of count site } \\
\text { attribution }\end{array}$ & 3 \\
\hline 01089010HU & 30 & 89 & Significant intersecting highway AADT & Install count site & 2 \\
\hline $01100050 \mathrm{HA}$ & 10 & 100 & No current data collection & Install count site & 1 \\
\hline 01101010HA & 10 & 101 & No current data collection & Install count site & 1 \\
\hline $01101010 \mathrm{HA}$ & 20 & 101 & No current data collection & Install count site & 1 \\
\hline $01101050 \mathrm{HA}$ & 10 & 101 & No current data collection & Install count site & 1 \\
\hline 01101060HA & 10 & 101 & No current data collection & Install count site & 1 \\
\hline 01190010HA & 20 & 190 & Near Centreport (Inland Port) & Consider short-duration count & 1 \\
\hline $01200050 \mathrm{HU}$ & 20 & 200 & Within city/town boundary (Winnipeg) & $\begin{array}{l}\text { Consider different method of count site } \\
\text { attribution }\end{array}$ & 3 \\
\hline $01200050 \mathrm{HU}$ & 30 & 200 & Within city/town boundary (Winnipeg) & $\begin{array}{l}\text { Consider different method of count site } \\
\text { attribution }\end{array}$ & 3 \\
\hline $01201060 \mathrm{HU}$ & 10 & 201 & Significant intersecting highway AADT & Install count site & 2 \\
\hline 01204030HU & 30 & 204 & Within city/town boundary (Selkirk) & $\begin{array}{l}\text { Consider different method of count site } \\
\text { attribution }\end{array}$ & 3 \\
\hline 01207010HU & 10 & 207 & No current data collection & Install count site & 1 \\
\hline 01207010HU & 20 & 207 & No current data collection & Install count site & 1 \\
\hline 01207015HA & 10 & 207 & No current data collection & Install count site & 1 \\
\hline 01207015HA & 20 & 207 & No current data collection & Install count site & 1 \\
\hline 01207017HA & 10 & 207 & No current data collection & Install count site & 1 \\
\hline 01207020HU & 10 & 207 & No current data collection & Install count site & 1 \\
\hline 01211010HU & 20 & 211 & Significant intersecting highway AADT & Install count site & 2 \\
\hline
\end{tabular}




\begin{tabular}{|c|c|c|c|c|c|}
\hline CS Key & Sequence & Highway & Concern & Recommendation & Priority \\
\hline $01212030 \mathrm{HU}$ & 20 & 212 & Significant intersecting highway AADT & Install count site & 2 \\
\hline 01246005HU & 10 & 246 & No current data collection & Install count site & 1 \\
\hline 01246005HU & 20 & 246 & $\begin{array}{l}\text { No current data collection on short segment } \\
\text { (connecting bridge removed) }\end{array}$ & $\begin{array}{l}\text { Update highway network LRS to exclude } \\
\text { this segment }\end{array}$ & 4 \\
\hline $01300010 \mathrm{HU}$ & 10 & 300 & No current data collection & Install count site & 1 \\
\hline $01300010 \mathrm{HU}$ & 20 & 300 & No current data collection & Install count site & 1 \\
\hline 01320010HA & 10 & 320 & Within city/town boundary (Selkirk) & $\begin{array}{l}\text { Consider different method of count site } \\
\text { attribution }\end{array}$ & 3 \\
\hline 01320015HU & 10 & 320 & Within city/town boundary (Selkirk) & $\begin{array}{l}\text { Consider different method of count site } \\
\text { attribution }\end{array}$ & 3 \\
\hline 01404010HU & 10 & 404 & Significant intersecting highway AADT & Install count site & 2 \\
\hline 02001930HA & 20 & 1 & $\begin{array}{l}\text { Within city/town boundary (Portage la } \\
\text { Prairie) }\end{array}$ & $\begin{array}{l}\text { Consider different method of count site } \\
\text { attribution }\end{array}$ & 3 \\
\hline 02001932HU & 10 & 1 & $\begin{array}{l}\text { Within city/town boundary (Portage la } \\
\text { Prairie) }\end{array}$ & $\begin{array}{l}\text { Consider different method of count site } \\
\text { attribution }\end{array}$ & 3 \\
\hline 02001935HA & 10 & 1 & $\begin{array}{l}\text { Within city/town boundary (Portage la } \\
\text { Prairie) }\end{array}$ & $\begin{array}{l}\text { Consider different method of count site } \\
\text { attribution }\end{array}$ & 3 \\
\hline 02001935HA & 20 & 1 & $\begin{array}{l}\text { Within city/town boundary (Portage la } \\
\text { Prairie) }\end{array}$ & $\begin{array}{l}\text { Consider different method of count site } \\
\text { attribution }\end{array}$ & 3 \\
\hline 02001935HA & 30 & 1 & $\begin{array}{l}\text { Within city/town boundary (Portage la } \\
\text { Prairie) }\end{array}$ & $\begin{array}{l}\text { Consider different method of count site } \\
\text { attribution }\end{array}$ & 3 \\
\hline 02001937HU & 10 & 1 & $\begin{array}{l}\text { Within city/town boundary (Portage la } \\
\text { Prairie) }\end{array}$ & $\begin{array}{l}\text { Consider different method of count site } \\
\text { attribution }\end{array}$ & 3 \\
\hline 02003180HU & 30 & 3 & Within city/town boundary (Morden) & $\begin{array}{l}\text { Consider different method of count site } \\
\text { attribution }\end{array}$ & 3 \\
\hline 02003180HU & 40 & 3 & Within city/town boundary (Morden) & $\begin{array}{l}\text { Consider different method of count site } \\
\text { attribution }\end{array}$ & 3 \\
\hline 02003180HU & 50 & 3 & Within city/town boundary (Morden) & $\begin{array}{l}\text { Consider different method of count site } \\
\text { attribution }\end{array}$ & 3 \\
\hline 02003185HA & 10 & 3 & Within city/town boundary (Morden) & $\begin{array}{l}\text { Consider different method of count site } \\
\text { attribution }\end{array}$ & 3 \\
\hline 02009080HU & 20 & 9 & Significant intersecting highway AADT & Install count site & 2 \\
\hline 02009090HU & 10 & 9 & Significant intersecting highway AADT & Install count site & 2 \\
\hline 02009090HU & 20 & 9 & Significant intersecting highway AADT & Install count site & 2 \\
\hline
\end{tabular}




\begin{tabular}{|c|c|c|c|c|c|}
\hline CS Key & Sequence & Highway & Concern & Recommendation & Priority \\
\hline 02009090HU & 30 & 9 & Significant intersecting highway AADT & Install count site & 2 \\
\hline 02009090HU & 40 & 9 & Significant intersecting highway AADT & Install count site & 2 \\
\hline 02014010HA & 10 & 14 & No current data collection & Install count site & 2 \\
\hline 02014010HA & 20 & 14 & Within city/town boundary (Winkler) & $\begin{array}{l}\text { Consider different method of count site } \\
\text { attribution }\end{array}$ & 3 \\
\hline 02014010HA & 30 & 14 & Within city/town boundary (Winkler) & $\begin{array}{l}\text { Consider different method of count site } \\
\text { attribution }\end{array}$ & 3 \\
\hline 02014020HA & 10 & 14 & Within city/town boundary (Winkler) & $\begin{array}{l}\text { Consider different method of count site } \\
\text { attribution }\end{array}$ & 3 \\
\hline 02014025HU & 10 & 14 & Within city/town boundary (Winkler) & $\begin{array}{l}\text { Consider different method of count site } \\
\text { attribution }\end{array}$ & 3 \\
\hline 02023090HU & 30 & 23 & Significant intersecting highway AADT & Install count site & 2 \\
\hline 02023090HU & 40 & 23 & Significant intersecting highway AADT & Install count site & 2 \\
\hline 02023100HU & 50 & 23 & Significant intersecting highway AADT & Install count site & 2 \\
\hline 02030010HU & 40 & 30 & Significant intersecting highway AADT & Install count site & 2 \\
\hline 02032010HU & 10 & 32 & Significant intersecting highway AADT & Install count site & 2 \\
\hline 02032010HU & 30 & 32 & Significant intersecting highway AADT & Install count site & 2 \\
\hline 02032010HU & 40 & 32 & Significant intersecting highway AADT & Install count site & 2 \\
\hline 02032010HU & 50 & 32 & Significant intersecting highway AADT & Install count site & 2 \\
\hline 02032010HU & 90 & 32 & Within city/town boundary (Winkler) & $\begin{array}{l}\text { Consider different method of count site } \\
\text { attribution }\end{array}$ & 3 \\
\hline 02067010HU & 20 & 67 & Significant intersecting highway AADT & Install count site & 2 \\
\hline 02068080HU & 10 & 68 & Significant intersecting highway AADT & Install count site & 2 \\
\hline 02240050HU & 30 & 240 & $\begin{array}{l}\text { Within city/town boundary (Portage la } \\
\text { Prairie) }\end{array}$ & $\begin{array}{l}\text { Consider different method of count site } \\
\text { attribution }\end{array}$ & 3 \\
\hline 02240070HU & 10 & 240 & $\begin{array}{l}\text { Within city/town boundary (Portage la } \\
\text { Prairie) }\end{array}$ & $\begin{array}{l}\text { Consider different method of count site } \\
\text { attribution }\end{array}$ & 3 \\
\hline 02240080HU & 10 & 240 & $\begin{array}{l}\text { Within city/town boundary (Portage la } \\
\text { Prairie) }\end{array}$ & $\begin{array}{l}\text { Consider different method of count site } \\
\text { attribution }\end{array}$ & 3 \\
\hline $02242080 \mathrm{HU}$ & 10 & 242 & Significant intersecting highway AADT & Install count site & 2 \\
\hline 02245020HU & 20 & 245 & Significant intersecting highway AADT & Install count site & 2 \\
\hline 02247020HU & 10 & 247 & Significant intersecting highway AADT & Install count site & 2 \\
\hline 02247020HU & 30 & 247 & Significant intersecting highway AADT & Install count site & 2 \\
\hline
\end{tabular}




\begin{tabular}{|c|c|c|c|c|c|}
\hline CS Key & Sequence & Highway & Concern & Recommendation & Priority \\
\hline 02247020HU & 40 & 247 & Significant intersecting highway AADT & Install count site & 2 \\
\hline 02248040HU & 50 & 248 & Significant intersecting highway AADT & Install count site & 2 \\
\hline 02305050HU & 10 & 305 & Significant intersecting highway AADT & Install count site & 2 \\
\hline 02321010HU & 10 & 321 & Significant intersecting highway AADT & Install count site & 2 \\
\hline 02321010HU & 20 & 321 & Significant intersecting highway AADT & Install count site & 2 \\
\hline 02322010HU & 10 & 322 & Significant intersecting highway AADT & Install count site & 2 \\
\hline 02322010HU & 20 & 322 & Significant intersecting highway AADT & Install count site & 2 \\
\hline 02332030HU & 20 & 332 & Significant intersecting highway AADT & Install count site & 2 \\
\hline 02334015HU & 10 & 334 & Significant intersecting highway AADT & Install count site & 2 \\
\hline 02336010HU & 20 & 336 & Significant intersecting highway AADT & Install count site & 2 \\
\hline 02411010HU & 20 & 411 & Significant intersecting highway AADT & Install count site & 2 \\
\hline 02415020HU & 10 & 415 & Significant intersecting highway AADT & Install count site & 2 \\
\hline 02428010HU & 10 & 428 & Within city/town boundary (Winkler) & $\begin{array}{l}\text { Consider different method of count site } \\
\text { attribution }\end{array}$ & 3 \\
\hline 02432010HU & 30 & 432 & Within city/town boundary (Morden) & $\begin{array}{l}\text { Consider different method of count site } \\
\text { attribution }\end{array}$ & 3 \\
\hline 02432010HU & 40 & 432 & Within city/town boundary (Morden) & $\begin{array}{l}\text { Consider different method of count site } \\
\text { attribution }\end{array}$ & 3 \\
\hline 03001090HA & 10 & 1 & Within city/town boundary (Brandon) & $\begin{array}{l}\text { Consider different method of count site } \\
\text { attribution }\end{array}$ & 3 \\
\hline 03001100HA & 10 & 1 & Within city/town boundary (Brandon) & $\begin{array}{l}\text { Consider different method of count site } \\
\text { attribution }\end{array}$ & 3 \\
\hline 03001910HU & 20 & 1 & Within city/town boundary (Brandon) & $\begin{array}{l}\text { Consider different method of count site } \\
\text { attribution }\end{array}$ & 3 \\
\hline 03001915HA & 10 & 1 & Within city/town boundary (Brandon) & $\begin{array}{l}\text { Consider different method of count site } \\
\text { attribution }\end{array}$ & 3 \\
\hline 03001920HA & 10 & 1 & Within city/town boundary (Brandon) & $\begin{array}{l}\text { Consider different method of count site } \\
\text { attribution }\end{array}$ & 3 \\
\hline 03001920HA & 20 & 1 & Within city/town boundary (Brandon) & $\begin{array}{l}\text { Consider different method of count site } \\
\text { attribution }\end{array}$ & 3 \\
\hline 03001920HA & 30 & 1 & Within city/town boundary (Brandon) & $\begin{array}{l}\text { Consider different method of count site } \\
\text { attribution }\end{array}$ & 3 \\
\hline
\end{tabular}




\begin{tabular}{|c|c|c|c|c|c|}
\hline CS Key & Sequence & Highway & Concern & Recommendation & Priority \\
\hline 03001925HU & 10 & 1 & Within city/town boundary (Brandon) & $\begin{array}{l}\text { Consider different method of count site } \\
\text { attribution }\end{array}$ & 3 \\
\hline 03003020HU & 20 & 3 & Significant intersecting highway AADT & Install count site & 2 \\
\hline 03003910HU & 10 & 3 & Significant intersecting highway AADT & Install count site & 2 \\
\hline $03010062 \mathrm{HU}$ & 20 & 10 & Within city/town boundary (Brandon) & $\begin{array}{l}\text { Consider different method of count site } \\
\text { attribution }\end{array}$ & 3 \\
\hline 03010065HA & 10 & 10 & Within city/town boundary (Brandon) & $\begin{array}{l}\text { Consider different method of count site } \\
\text { attribution }\end{array}$ & 3 \\
\hline 03010065HA & 20 & 10 & Within city/town boundary (Brandon) & $\begin{array}{l}\text { Consider different method of count site } \\
\text { attribution }\end{array}$ & 3 \\
\hline 03010070HA & 10 & 10 & Within city/town boundary (Brandon) & $\begin{array}{l}\text { Consider different method of count site } \\
\text { attribution }\end{array}$ & 3 \\
\hline 03010075HU & 10 & 10 & Within city/town boundary (Brandon) & $\begin{array}{l}\text { Consider different method of count site } \\
\text { attribution }\end{array}$ & 3 \\
\hline 03010080HU & 10 & 10 & $\begin{array}{l}\text { Traffic source/sink at seqeuncing location } \\
\text { (Brandon Airport) }\end{array}$ & Install count site & 2 \\
\hline 03010114HU & 20 & 10 & Significant intersecting highway AADT & Install count site & 2 \\
\hline 03010116HU & 10 & 10 & Significant intersecting highway AADT & Install count site & 2 \\
\hline 03016070HU & 10 & 16 & Significant intersecting highway AADT & Install count site & 2 \\
\hline 03023060HU & 30 & 23 & Significant intersecting highway AADT & Install count site & 2 \\
\hline 03024050HU & 10 & 24 & Significant intersecting highway AADT & Install count site & 2 \\
\hline 03025010HU & 10 & 25 & Significant intersecting highway AADT & Install count site & 2 \\
\hline 03041020HU & 10 & 41 & Significant intersecting highway AADT & Install count site & 2 \\
\hline 03045060HU & 10 & 45 & Significant intersecting highway AADT & Install count site & 2 \\
\hline 03045060HU & 20 & 45 & Significant intersecting highway AADT & Install count site & 2 \\
\hline $03083010 \mathrm{HU}$ & 20 & 83 & Significant intersecting highway AADT & Install count site & 2 \\
\hline $03110010 \mathrm{HU}$ & 20 & 110 & Within city/town boundary (Brandon) & $\begin{array}{l}\text { Consider different method of count site } \\
\text { attribution }\end{array}$ & 3 \\
\hline 03110020HU & 10 & 110 & Within city/town boundary (Brandon) & $\begin{array}{l}\text { Consider different method of count site } \\
\text { attribution }\end{array}$ & 3 \\
\hline $03110020 \mathrm{HU}$ & 40 & 110 & Significant intersecting highway AADT & Install count site & 2 \\
\hline 03257010HU & 20 & 257 & Significant intersecting highway AADT & Install count site & 2 \\
\hline $03257030 \mathrm{HU}$ & 10 & 30 & Significant intersecting highway AADT & Install count site & 1 \\
\hline
\end{tabular}




\begin{tabular}{|c|c|c|c|c|c|}
\hline CS Key & Sequence & Highway & Concern & Recommendation & Priority \\
\hline $03257030 \mathrm{HU}$ & 20 & 30 & Significant intersecting highway AADT & Install count site & 1 \\
\hline 03259040HU & 50 & 40 & Significant intersecting highway AADT & Install count site & 1 \\
\hline $03342010 \mathrm{HU}$ & 20 & 342 & Significant intersecting highway AADT & Install count site & 2 \\
\hline $03344015 \mathrm{HU}$ & 10 & 344 & $\begin{array}{l}\text { No current data collection (connecting } \\
\text { bridge removed) }\end{array}$ & Install count site & 1 \\
\hline $03344015 \mathrm{HU}$ & 20 & 344 & No current data collection & Install count site & 1 \\
\hline 03457010HU & 10 & 457 & Within city/town boundary (Brandon) & $\begin{array}{l}\text { Consider different method of count site } \\
\text { attribution }\end{array}$ & 3 \\
\hline $03459010 \mathrm{HU}$ & 30 & 459 & Within city/town boundary (Brandon) & $\begin{array}{l}\text { Consider different method of count site } \\
\text { attribution }\end{array}$ & 3 \\
\hline $03530015 \mathrm{HU}$ & 10 & 530 & $\begin{array}{l}\text { No current data collection (connecting } \\
\text { bridge removed) }\end{array}$ & Install count site & 1 \\
\hline 04005260HU & 40 & 5 & Significant intersecting highway AADT & Install count site & 2 \\
\hline 04005910HU & 10 & 5 & No current data collection & Install count site & 2 \\
\hline 04005910HU & 20 & 5 & Within city/town boundary (Dauphin) & $\begin{array}{l}\text { Consider different method of count site } \\
\text { attribution }\end{array}$ & 3 \\
\hline 04005920HU & 10 & 5 & Within city/town boundary (Dauphin) & $\begin{array}{l}\text { Consider different method of count site } \\
\text { attribution }\end{array}$ & 3 \\
\hline 04005930HU & 10 & 5 & Within city/town boundary (Dauphin) & $\begin{array}{l}\text { Consider different method of count site } \\
\text { attribution }\end{array}$ & 3 \\
\hline 04005930HU & 20 & 5 & Within city/town boundary (Dauphin) & $\begin{array}{l}\text { Consider different method of count site } \\
\text { attribution }\end{array}$ & 3 \\
\hline 04010920HU & 10 & 10 & Significant intersecting highway AADT & Install count site & 2 \\
\hline 04010930HU & 10 & 10 & Significant intersecting highway AADT & Install count site & 2 \\
\hline 04020030HU & 10 & 20 & Within city/town boundary (Dauphin) & $\begin{array}{l}\text { Consider different method of count site } \\
\text { attribution }\end{array}$ & 3 \\
\hline 04020910HU & 10 & 20 & Within city/town boundary (Dauphin) & $\begin{array}{l}\text { Consider different method of count site } \\
\text { attribution }\end{array}$ & 3 \\
\hline 04020920HU & 10 & 20 & Within city/town boundary (Dauphin) & $\begin{array}{l}\text { Consider different method of count site } \\
\text { attribution }\end{array}$ & 3 \\
\hline 04083120HU & 10 & 83 & Significant intersecting highway AADT & Install count site & 2 \\
\hline 04083910HU & 10 & 83 & Significant intersecting highway AADT & Install count site & 2 \\
\hline $04266010 \mathrm{HU}$ & 10 & 266 & No current data collection & Install count site & 1 \\
\hline
\end{tabular}




\begin{tabular}{|c|c|c|c|c|c|}
\hline CS Key & Sequence & Highway & Concern & Recommendation & Priority \\
\hline 04362010HU & 10 & 362 & Within city/town boundary (Dauphin) & $\begin{array}{l}\text { Consider different method of count site } \\
\text { attribution }\end{array}$ & 3 \\
\hline $04363010 \mathrm{HU}$ & 10 & 363 & Significant intersecting highway AADT & Install count site & 2 \\
\hline $04417010 \mathrm{HU}$ & 20 & 417 & Significant intersecting highway AADT & Install count site & 2 \\
\hline $05006265 \mathrm{HA}$ & 10 & 6 & Within city/town boundary (Thompson) & $\begin{array}{l}\text { Consider different method of count site } \\
\text { attribution }\end{array}$ & 3 \\
\hline $05010310 \mathrm{HU}$ & 20 & 10 & Within city/town boundary (The Pas) & $\begin{array}{l}\text { Consider different method of count site } \\
\text { attribution }\end{array}$ & 3 \\
\hline $05010370 \mathrm{HU}$ & 60 & 10 & Within city/town boundary (Flin Flon) & $\begin{array}{l}\text { Consider different method of count site } \\
\text { attribution }\end{array}$ & 3 \\
\hline $05010380 \mathrm{HU}$ & 10 & 10 & Within city/town boundary (Flin Flon) & $\begin{array}{l}\text { Consider different method of count site } \\
\text { attribution }\end{array}$ & 3 \\
\hline $05010380 \mathrm{HU}$ & 30 & 10 & Within city/town boundary (Flin Flon) & $\begin{array}{l}\text { Consider different method of count site } \\
\text { attribution }\end{array}$ & 3 \\
\hline 05010390HU & 10 & 10 & Within city/town boundary (Flin Flon) & $\begin{array}{l}\text { Consider different method of count site } \\
\text { attribution }\end{array}$ & 3 \\
\hline 05010940HU & 20 & 10 & Within city/town boundary (Flin Flon) & $\begin{array}{l}\text { Consider different method of count site } \\
\text { attribution }\end{array}$ & 3 \\
\hline 05039050EU & 20 & 39 & No current data collection & Install count site & 1 \\
\hline $05283020 \mathrm{HU}$ & 30 & 283 & Within city/town boundary (The Pas) & $\begin{array}{l}\text { Consider different method of count site } \\
\text { attribution }\end{array}$ & 3 \\
\hline 05285010HU & 10 & 285 & Significant intersecting highway AADT & Install count site & 2 \\
\hline 05285010HU & 30 & 285 & Within city/town boundary (The Pas) & $\begin{array}{l}\text { Consider different method of count site } \\
\text { attribution }\end{array}$ & 3 \\
\hline 05287010HU & 20 & 287 & Within city/town boundary (The Pas) & $\begin{array}{l}\text { Consider different method of count site } \\
\text { attribution }\end{array}$ & 3 \\
\hline 05289010HU & 20 & 289 & Within city/town boundary (The Pas) & $\begin{array}{l}\text { Consider different method of count site } \\
\text { attribution }\end{array}$ & 3 \\
\hline 05291010HU & 10 & 291 & Within city/town boundary (Flin Flon) & $\begin{array}{l}\text { Consider different method of count site } \\
\text { attribution }\end{array}$ & 3 \\
\hline 05291010HU & 20 & 291 & Within city/town boundary (Flin Flon) & $\begin{array}{l}\text { Consider different method of count site } \\
\text { attribution }\end{array}$ & 3 \\
\hline 05291010HU & 30 & 291 & Within city/town boundary (Flin Flon) & $\begin{array}{l}\text { Consider different method of count site } \\
\text { attribution }\end{array}$ & 3 \\
\hline
\end{tabular}




\begin{tabular}{|c|r|r|l|l|}
\hline CS Key & Sequence & Highway & \multicolumn{1}{|c|}{ Concern } & Recommendation \\
\hline $\mathbf{0 5 3 7 4 0 1 0 H U}$ & 10 & 374 & No current data collection & Install count site \\
\hline $\mathbf{0 5 3 8 4 0 1 0 H U}$ & 10 & 384 & No current data collection & Install count site \\
\hline $\mathbf{0 5 3 9 1 0 1 0 H A}$ & 10 & 391 & Within city/town boundary (Thompson) & $\begin{array}{l}\text { Consider different method of count site } \\
\text { attribution }\end{array}$ \\
\hline $\mathbf{0 5 3 9 1 0 1 5 H U}$ & 10 & 391 & Within city/town boundary (Thompson) & $\begin{array}{l}\text { Consider different method of count site } \\
\text { attribution }\end{array}$ \\
\hline $\mathbf{0 5 4 9 3 0 1 0 H U}$ & 10 & 493 & Only count on segment is a town count & $\begin{array}{l}\text { Install count site (continuous or short- } \\
\text { duration) }\end{array}$ \\
\hline $\mathbf{0 5 4 9 3 0 2 0 H U}$ & 10 & 493 & Only count on segment is a town count & $\begin{array}{l}\text { Install count site (continuous or short- } \\
\text { duration) }\end{array}$ \\
\hline $\mathbf{0 5 4 9 3 0 3 0 H U}$ & 10 & 493 & Only count on segment is a town count & $\begin{array}{l}\text { Install count site (continuous or short- } \\
\text { duration) }\end{array}$ \\
\hline
\end{tabular}




\section{APPENDIX E: REDUNDANT COUNT SITES}

\begin{tabular}{|c|c|c|c|}
\hline Station & Highway & Concern & Recommendation \\
\hline 72 & 10 & Redundant (PCS, 72 is an AVC) & Consider removing \\
\hline 149 & 12 & $\begin{array}{l}\text { Within city/town boundary } \\
\text { (Steinbach) }\end{array}$ & $\begin{array}{l}\text { Redesignate to Town Count, } \\
\text { move, or remove }\end{array}$ \\
\hline 150 & 52 & $\begin{array}{l}\text { Within city/town boundary } \\
\text { (Steinbach) }\end{array}$ & $\begin{array}{l}\text { Redesignate to Town Count, } \\
\text { move, or remove }\end{array}$ \\
\hline 151 & 52 & $\begin{array}{l}\text { Within city/town boundary } \\
\text { (Steinbach) }\end{array}$ & $\begin{array}{l}\text { Redesignate to Town Count, } \\
\text { move, or remove }\end{array}$ \\
\hline 187 & 17 & Redundant & Remove \\
\hline 194 & 68 & Redundant & Remove \\
\hline 219 & 68 & Redundant & Remove \\
\hline 243 & 14 & $\begin{array}{l}\text { Within city/town boundary } \\
\text { (Winkler) }\end{array}$ & $\begin{array}{l}\text { Redesignate to Town Count, } \\
\text { move, or remove }\end{array}$ \\
\hline 245 & 3 & Redundant & Remove \\
\hline 264 & 34 & Redundant & Remove \\
\hline 282 & 18 & Redundant & Remove \\
\hline 305 & 10 & Redundant & Remove \\
\hline 336 & 255 & Redundant & Remove \\
\hline 349 & 1 & Redundant & Remove \\
\hline 366 & 240 & $\begin{array}{l}\text { Within city/town boundary } \\
\text { (Portage la Prairie) }\end{array}$ & $\begin{array}{l}\text { Redesignate to Town Count, } \\
\text { move, or remove }\end{array}$ \\
\hline 375 & 1 & Redundant & Remove \\
\hline 376 & 26 & $\begin{array}{l}\text { Within city/town boundary (Saint } \\
\text { Francois Xavier) }\end{array}$ & $\begin{array}{l}\text { Redesignate to Town Count, } \\
\text { move, or remove }\end{array}$ \\
\hline 403 & 21 & Redundant & Remove \\
\hline 435 & 259 & Redundant & Remove \\
\hline 464 & 5 & Redundant & Remove \\
\hline 474 & 20 & Redundant & Remove \\
\hline 482 & 20 & Redundant & Remove \\
\hline 501 & 10 & $\begin{array}{l}\text { Within city/town boundary (Flin } \\
\text { Flon) }\end{array}$ & $\begin{array}{l}\text { Redesignate to Town Count, } \\
\text { move, or remove }\end{array}$ \\
\hline 510 & 7 & Redundant & Remove \\
\hline 511 & 1 & $\begin{array}{l}\text { Within city/town boundary } \\
\text { (Brandon) }\end{array}$ & $\begin{array}{l}\text { Redesignate to Town Count, } \\
\text { move, or remove }\end{array}$ \\
\hline 531 & 304 & Redundant & Remove \\
\hline 554 & 367 & Redundant & Remove \\
\hline 563 & 9 & $\begin{array}{l}\text { Within city/town boundary } \\
\text { (Selkirk) but outside urban centre }\end{array}$ & $\begin{array}{l}\text { Monitor for future growth and } \\
\text { redesignate to Town Count, } \\
\text { move, or remove when necessary }\end{array}$ \\
\hline 564 & 9 & $\begin{array}{l}\text { Within city/town boundary } \\
\text { (Selkirk) }\end{array}$ & $\begin{array}{l}\text { Redesignate to Town Count, } \\
\text { move, or remove }\end{array}$ \\
\hline 565 & 9 & $\begin{array}{l}\text { Within city/town boundary } \\
\text { (Selkirk) }\end{array}$ & $\begin{array}{l}\text { Redesignate to Town Count, } \\
\text { move, or remove }\end{array}$ \\
\hline 568 & 204 & $\begin{array}{l}\text { Within city/town boundary } \\
\text { (Selkirk) but outside urban centre }\end{array}$ & $\begin{array}{l}\text { Monitor for future growth and } \\
\text { redesignate to Town Count, } \\
\text { move, or remove when necessary }\end{array}$ \\
\hline 578 & 5 & Redundant & Remove \\
\hline 587 & 8 & Redundant & Remove \\
\hline 588 & 44 & Redundant & Remove \\
\hline
\end{tabular}




\begin{tabular}{|c|c|c|c|}
\hline Station & Highway & Concern & Recommendation \\
\hline 604 & 8 & Redundant & Remove \\
\hline 609 & 17 & Redundant & Remove \\
\hline 622 & 251 & Redundant & Remove \\
\hline 624 & 256 & Redundant & Remove \\
\hline 647 & 2 & Redundant & Remove \\
\hline 673 & 100 & Redundant & Remove \\
\hline 684 & 307 & Redundant & Remove \\
\hline 689 & 17 & Redundant & Remove \\
\hline 734 & 243 & Redundant & Remove \\
\hline 742 & 308 & Redundant & Remove \\
\hline 749 & 302 & Redundant & Remove \\
\hline 762 & 203 & Redundant & Remove \\
\hline 763 & 210 & Redundant & Remove \\
\hline 766 & 203 & Redundant & Remove \\
\hline 767 & 404 & Redundant & Remove \\
\hline 768 & 210 & Redundant & Remove \\
\hline 788 & 217 & Redundant & Remove \\
\hline 789 & 200 & Redundant & Remove \\
\hline 792 & 217 & Redundant & Remove \\
\hline 804 & 216 & Redundant & Remove \\
\hline 854 & 503 & Redundant & Remove \\
\hline 882 & 408 & Redundant & Remove \\
\hline 890 & 212 & Redundant & Remove \\
\hline 925 & 201 & Redundant & Remove \\
\hline 935 & 201 & Redundant & Remove \\
\hline 950 & 432 & $\begin{array}{l}\text { Within city/town boundary } \\
\text { (Morden) }\end{array}$ & $\begin{array}{l}\text { Redesignate to Town Count, } \\
\text { move, or remove }\end{array}$ \\
\hline 957 & 240 & Redundant & Remove \\
\hline 962 & 422 & Redundant & Remove \\
\hline 963 & 332 & Redundant & Remove \\
\hline 966 & 336 & Redundant & Remove \\
\hline 985 & 242 & $\begin{array}{l}\text { Within city/town boundary } \\
\text { (Somerset) }\end{array}$ & $\begin{array}{l}\text { Redesignate to Town Count, } \\
\text { move, or remove }\end{array}$ \\
\hline 1003 & 242 & Redundant & Remove \\
\hline 1004 & 449 & Redundant & Remove \\
\hline 1065 & 352 & Redundant & Remove \\
\hline 1088 & 67 & Redundant & Remove \\
\hline 1114 & 227 & Redundant & Remove \\
\hline 1130 & 411 & Redundant & Remove \\
\hline 1158 & 8 & Redundant & Remove \\
\hline 1161 & 234 & Redundant & Remove \\
\hline 1206 & 329 & Redundant & Remove \\
\hline 1207 & 233 & Redundant & Remove \\
\hline 1240 & 442 & Redundant & Remove \\
\hline 1243 & 342 & Redundant & Remove \\
\hline 1248 & 5 & Redundant & Remove \\
\hline 1282 & 341 & Redundant & Remove \\
\hline 1324 & 256 & Redundant & Remove \\
\hline
\end{tabular}




\begin{tabular}{|c|c|c|c|}
\hline Station & Highway & Concern & Recommendation \\
\hline 1345 & 256 & Redundant & Remove \\
\hline 1346 & 256 & Redundant & Remove \\
\hline 1368 & 250 & Redundant & Remove \\
\hline 1396 & 10 & $\begin{array}{l}\text { Within city/town boundary } \\
\text { (Brandon) }\end{array}$ & $\begin{array}{l}\text { Redesignate to Town Count, } \\
\text { move, or remove }\end{array}$ \\
\hline 1417 & 265 & Redundant & Remove \\
\hline 1443 & 353 & Redundant & Remove \\
\hline 1465 & 357 & Redundant & Remove \\
\hline 1469 & 270 & Redundant & Remove \\
\hline 1470 & 270 & Redundant & Remove \\
\hline 1494 & 256 & Redundant & Remove \\
\hline 1511 & 270 & Redundant & Remove \\
\hline 1517 & 354 & Redundant & Remove \\
\hline 1523 & 355 & Redundant & Remove \\
\hline 1525 & 264 & Redundant & Remove \\
\hline 1535 & 270 & Redundant & Remove \\
\hline 1537 & 250 & Redundant & Remove \\
\hline 1559 & 354 & Redundant & Remove \\
\hline 1570 & 354 & Redundant & Remove \\
\hline 1573 & 577 & Redundant & Remove \\
\hline 1586 & 579 & Redundant & Remove \\
\hline 1592 & 478 & Redundant & Remove \\
\hline 1605 & 462 & Redundant & Remove \\
\hline 1609 & 261 & Redundant & Remove \\
\hline 1614 & 261 & Redundant & Remove \\
\hline 1623 & 360 & Redundant & Remove \\
\hline 1628 & 68 & Redundant & Remove \\
\hline 1631 & 276 & Redundant & Remove \\
\hline 1643 & 276 & Redundant & Remove \\
\hline 1662 & 584 & Redundant & Remove \\
\hline 1675 & 482 & Redundant & Remove \\
\hline 1686 & 364 & Redundant & Remove \\
\hline 1690 & 364 & Redundant & Remove \\
\hline 1693 & 271 & Redundant & Remove \\
\hline 1694 & 489 & Redundant & Remove \\
\hline 1696 & 271 & Redundant & Remove \\
\hline 1729 & 487 & Redundant & Remove \\
\hline 1737 & 275 & Redundant & Remove \\
\hline 1764 & 512 & Redundant & Remove \\
\hline 1765 & 68 & Redundant & Remove \\
\hline 1804 & 406 & Redundant & Remove \\
\hline 1831 & 201 & Within city/town boundary & $\begin{array}{l}\text { Redesignate to Town Count, } \\
\text { move, or remove }\end{array}$ \\
\hline 1838 & 1 & $\begin{array}{l}\text { Within city/town boundary } \\
\text { (Brandon) }\end{array}$ & $\begin{array}{l}\text { Redesignate to Town Count, } \\
\text { move, or remove }\end{array}$ \\
\hline 1839 & 1 & $\begin{array}{l}\text { Within city/town boundary } \\
\text { (Brandon) }\end{array}$ & $\begin{array}{l}\text { Redesignate to Town Count, } \\
\text { move, or remove }\end{array}$ \\
\hline 1892 & 60 & Redundant & Remove \\
\hline
\end{tabular}




\begin{tabular}{|c|c|c|c|}
\hline Station & Highway & Concern & Recommendation \\
\hline 1895 & 10 & Redundant & Remove \\
\hline 2011 & 391 & $\begin{array}{l}\text { Within city/town boundary } \\
\text { (Thompson) }\end{array}$ & $\begin{array}{l}\text { Redesignate to Town Count, } \\
\text { move, or remove }\end{array}$ \\
\hline 2027 & 240 & $\begin{array}{l}\text { Within city/town boundary } \\
\text { (Portage la Prairie) }\end{array}$ & $\begin{array}{l}\text { Redesignate to Town Count, } \\
\text { move, or remove }\end{array}$ \\
\hline 2037 & 68 & Redundant & Remove \\
\hline 2039 & 313 & Redundant & Remove \\
\hline 2055 & 584 & Redundant & Remove \\
\hline 2074 & 236 & Redundant & Remove \\
\hline 2075 & 255 & Redundant & Remove \\
\hline 2085 & 213 & Redundant & Remove \\
\hline 2088 & 260 & Redundant & Remove \\
\hline 2092 & 52 & $\begin{array}{l}\text { Within city/town boundary } \\
\text { (Steinbach) }\end{array}$ & $\begin{array}{l}\text { Redesignate to Town Count, } \\
\text { move, or remove }\end{array}$ \\
\hline 2097 & 283 & Redundant & Remove \\
\hline 2102 & 5 & Redundant & Remove \\
\hline 2104 & 242 & Redundant & Remove \\
\hline 2110 & 227 & Redundant & Remove \\
\hline 2130 & 487 & Redundant & Remove \\
\hline 2137 & 317 & Redundant & Remove \\
\hline 2138 & 229 & Redundant & Remove \\
\hline 2161 & 347 & Redundant & Remove \\
\hline 2162 & 443 & Redundant & Remove \\
\hline 2164 & 528 & Redundant & Remove \\
\hline 2168 & 326 & Redundant & Remove \\
\hline 2172 & 311 & Redundant & Remove \\
\hline 2173 & 216 & Redundant & Remove \\
\hline 2176 & 365 & Redundant & Remove \\
\hline 2177 & 210 & Redundant & Remove \\
\hline 2178 & 210 & Redundant & Remove \\
\hline 2196 & 246 & Redundant & Remove \\
\hline 2200 & 391 & Redundant & Remove \\
\hline 2201 & 10 & Redundant & Remove \\
\hline 2204 & 269 & Redundant & Remove \\
\hline 2206 & 278 & Redundant & Remove \\
\hline 2221 & 416 & Redundant & Remove \\
\hline 2256 & 455 & Redundant & Remove \\
\hline 2259 & 353 & Redundant & Remove \\
\hline 2260 & 236 & Redundant & Remove \\
\hline 2262 & 403 & Redundant & Remove \\
\hline 2276 & 261 & Redundant & Remove \\
\hline 2291 & 355 & Redundant & Remove \\
\hline 2302 & 246 & Redundant & Remove \\
\hline 2304 & 211 & Redundant & Remove \\
\hline 2309 & 332 & Redundant & Remove \\
\hline 2320 & 262 & Redundant & Remove \\
\hline 2332 & 305 & Redundant & Remove \\
\hline 2333 & 305 & Redundant & Remove \\
\hline
\end{tabular}




\begin{tabular}{|c|c|c|c|}
\hline Station & Highway & Concern & Recommendation \\
\hline 2352 & 430 & Redundant & Remove \\
\hline 2362 & 329 & Redundant & Remove \\
\hline 2387 & 500 & Redundant & Remove \\
\hline 2392 & 359 & Redundant & Remove \\
\hline 2396 & 218 & Redundant & Remove \\
\hline 2400 & 367 & Redundant & Remove \\
\hline 2401 & 367 & Redundant & Remove \\
\hline 2406 & 328 & Redundant & Remove \\
\hline 2407 & 328 & Redundant & Remove \\
\hline 2408 & 334 & Redundant & Remove \\
\hline 2425 & 10 & $\begin{array}{l}\text { Within city/town boundary (Flin } \\
\text { Flon) }\end{array}$ & $\begin{array}{l}\text { Redesignate to Town Count, } \\
\text { move, or remove }\end{array}$ \\
\hline 2448 & 302 & Redundant & Remove \\
\hline 2450 & 354 & Redundant & Remove \\
\hline 2458 & 20 & $\begin{array}{l}\text { Within city/town boundary } \\
\text { (Dauphin) }\end{array}$ & $\begin{array}{l}\text { Redesignate to Town Count, } \\
\text { move, or remove }\end{array}$ \\
\hline 2464 & 5 & $\begin{array}{l}\text { Within city/town boundary } \\
\text { (Dauphin) }\end{array}$ & $\begin{array}{l}\text { Redesignate to Town Count, } \\
\text { move, or remove }\end{array}$ \\
\hline 2467 & 474 & Redundant & Remove \\
\hline 2479 & 338 & Redundant & Remove \\
\hline 2480 & 549 & Redundant & Remove \\
\hline 2482 & 520 & Redundant & Remove \\
\hline 2500 & 4 & $\begin{array}{l}\text { Within city/town boundary } \\
\text { (Selkirk) but outside built up area }\end{array}$ & $\begin{array}{l}\text { Monitor for future growth and } \\
\text { redesignate to Town Count, } \\
\text { move, or remove when necessary }\end{array}$ \\
\hline 2511 & 10 & $\begin{array}{l}\text { Within city/town boundary (Flin } \\
\text { Flon) }\end{array}$ & $\begin{array}{l}\text { Redesignate to Town Count, } \\
\text { move, or remove }\end{array}$ \\
\hline 2514 & 475 & Redundant & Remove \\
\hline 2527 & 206 & Redundant & Remove \\
\hline 2530 & 23 & Redundant & Remove \\
\hline 2539 & 83 & Redundant & Remove \\
\hline 2542 & 10 & Redundant & Remove \\
\hline 2543 & 2 & Redundant & Remove \\
\hline 2547 & 355 & Redundant & Remove \\
\hline 2549 & 250 & Redundant & Remove \\
\hline 2553 & 247 & Redundant & Remove \\
\hline 2574 & 250 & Redundant & Remove \\
\hline 2579 & 59 & Redundant & Remove \\
\hline 6419 & 15 & Redundant & Remove \\
\hline
\end{tabular}

